\title{
Trace-Element Deposition in the Cariaco Basin, Venezuela Shelf, under Sulfate- Reducing Conditions-a History of the Local Hydrography and Global Climate, 20 ka to the Present
}

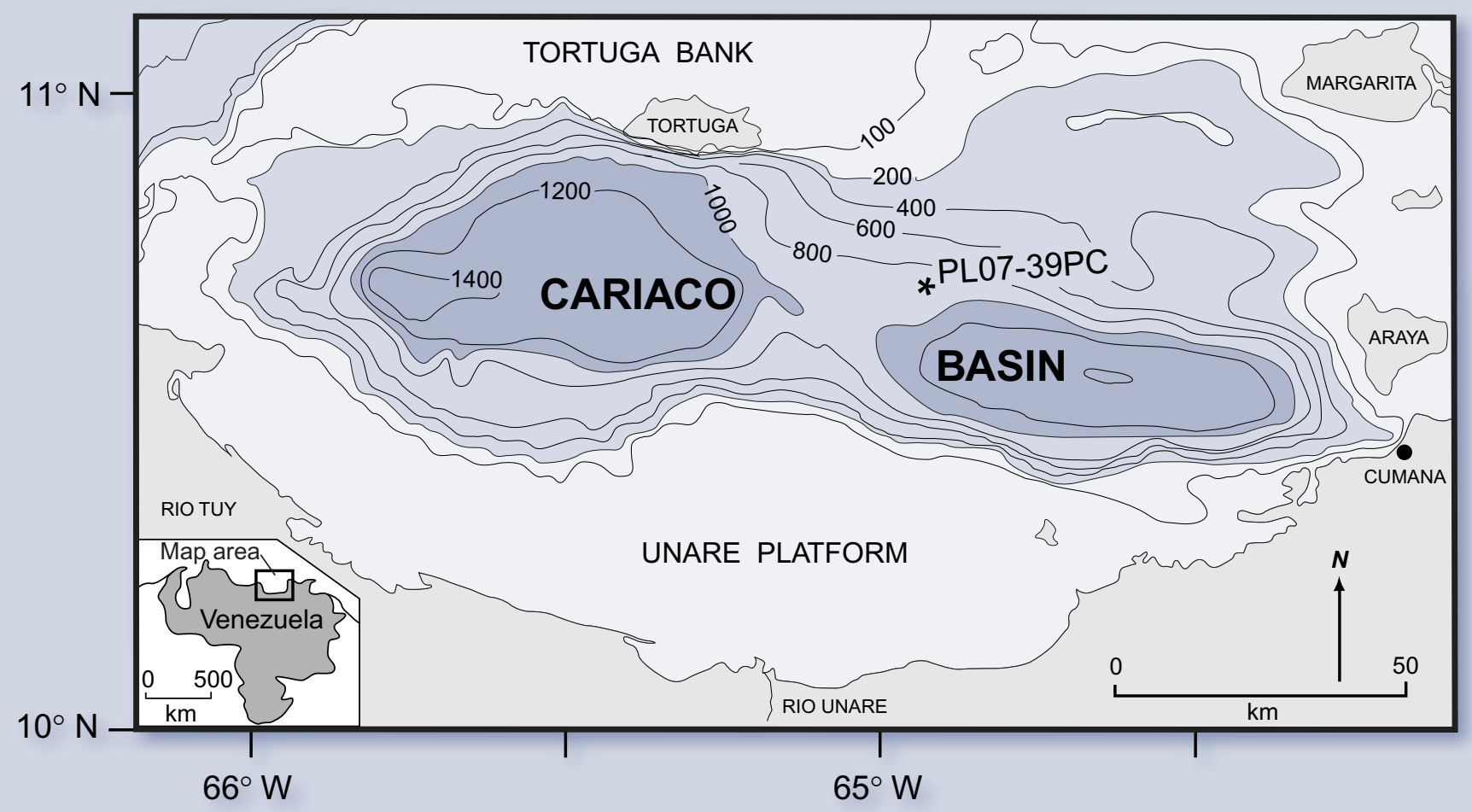

Professional Paper 1670 


\section{Trace-Element Deposition in the Cariaco Basin, Venezuela Shelf, under Sulfate- Reducing Conditions - a History of the Local Hydrography and Global Climate, 20 ka to the Present}

By David Z. Piper and Walter E. Dean

Professional Paper 1670 


\title{
U.S. Department of the Interior \\ Gale A. Norton, Secretary
}

\author{
U.S. Geological Survey \\ Charles G. Groat, Director
}

Any use of trade, product, or firm names in this publication is for descriptive purposes only and does not imply endorsement by the U.S. Government.

United States Government Printing Office: 2002

For additional copies please contact:

USGS Information Services

Box 25286

Denver, CO 80225

This report and any updates to it are available online at http://geopubs.wr.usgs.gov/prof-paper/pp1670/

Additional USGS publications can be found online at http://geology.usgs.gov/products.html

For more information about the USGS and its products:

Telephone: 1-888-ASK-USGS (1-888-275-8747)

World Wide Web: http://www.usgs.gov/

Text edited by James W. Hendley II Layout and design by Sara Boore

Manuscript approved for publication, 0ctober 22, 2002

Cataloging-in Publication data is on file with the Library of Congress 


\section{Contents}

Abstract...

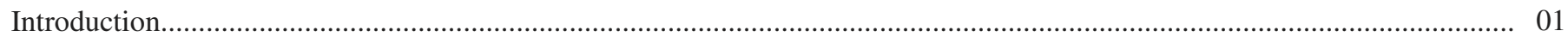

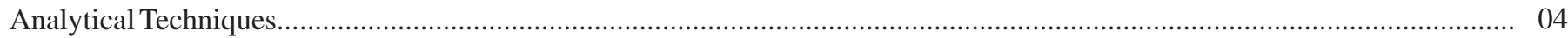

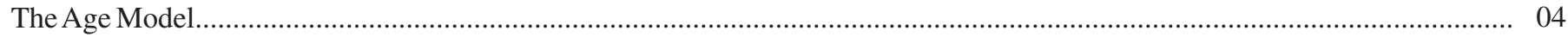

Partitioning Trace Elements between Terrigenous-Source and Seawater-Source Fractions-A Trace-Element Model.................. 04

Trace-Element Depositional Rates in the Surface Sediment—A Test of the Model............................................................ 28

Trace-Element Accumulation Rates during the Past 20,000 Years-An Application of the Model......................................... 30

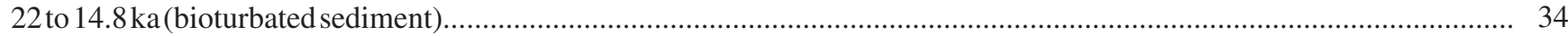

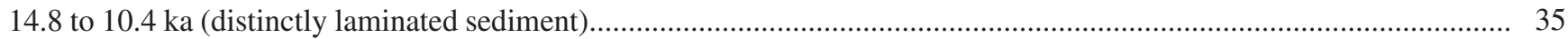

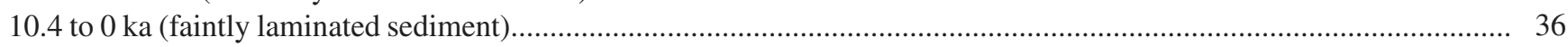

Elemental Ratios and Concentrations_-An Alternative Model of Environmental Conditions.................................................. 37

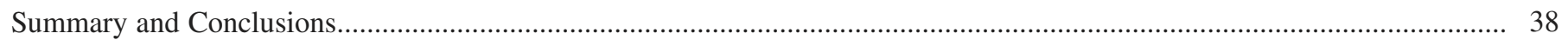

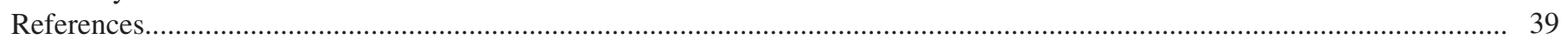

\section{Figures}

1. Bathymetry of the northwest continental shelf of Venezuela showing location of core PL07-39PC............................ 02

2. Concentrations of $\mathrm{Al}_{2} \mathrm{O}_{3}, \mathrm{Cd}, \mathrm{Mo}$, and $\mathrm{Zn}$ with depth in core PL07-39PC............................................................... 03

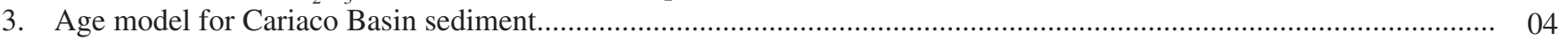

4. Relation between the concentrations of $\mathrm{Al}_{2} \mathrm{O}_{3}$ and $\mathrm{CaO}$ in core PL07-39PC...................................................... 13

5. Relations between the concentration of the terrigenous fraction, and $\mathrm{Al}_{2} \mathrm{O}_{3}$, and the concentrations of major-element oxides in core PL07-39PC.

6. Relations between the concentration of the terrigenous fraction and the concentrations of trace elements that exhibit approximate single trends with the terrigenous fraction in core PL07-39PC........................................... 22

7. Relation between the concentrations of the terrigenous fraction and manganese (Mn) in core PL07-39PC.................. 23

8. Relations between the concentration of the terrigenous fraction and the concentrations of Mo and $\mathrm{Cd}$ in core PL07-39PC.

9. Relations between the concentration of the terrigenous fraction and the concentrations of trace elements in core PL07-39PC that have strong factor loadings on the same factor as $\mathrm{Al}_{2} \mathrm{O}_{3}$, but have concentrations that are significantly greater than can be attributed solely to a terrigenous contribution......

10. Percentage of $\mathrm{Cd}, \mathrm{Cu}, \mathrm{Ni}$, and $\mathrm{Zn}$ and $\mathrm{Mo}$ and $\mathrm{V}$ removed from $\mathrm{SO}_{4}{ }^{2-}$-reducing bottom waters of modern marine basins....... 27

11. Schematic for the Cariaco Basin, showing "model" accumulation rates of the marine and terrigenous fractions of $\mathrm{Cd}$ and Mo.

12. Measured accumulation rates of the terrigenous and marine fractions of trace elements versus depth in core PL07-39PC.

13. Relations between the concentrations of $\mathrm{Mo}$ and $\mathrm{Zn}$ and $\mathrm{Mo}$ and $\mathrm{Cd}$ in the seawater-derived fractions of sediment in core PL07-39PC.

14. Relation between the concentrations of organic carbon and sediment depth in piston core PL07-39PC..................... 38

\section{Tables}

1. Concentrations in sediment of core P107-39PC of major-element oxides.

2. Concentrations of major-element oxides and trace elements (1) in the world shale average (WSA) and the terrigenous fraction of the Cariaco Basin, (2) in marine biogenic debris, and (3) in seawater.

3. Major components in sediment samples from the Cariaco Basin, in percent, calculated from major-oxide concentrations (table 1).

4. Correlation coefficients for major-element oxides in sediment from the Cariaco Basin...

5. Varimax orthogonal transformation solution, principal components factor extraction method, of the $\log 10$ of trace-element analyses and major sediment components in sediment core PL07-39PC, calculated from major-element-oxide analyses, using Statviewtm II software.

6. Concentrations of trace elements in seawater and organic matter in the Cariaco Basin and their rates of deposition in the surface sediment of core PL07-39PC.

7. Calculated rates of deposition for the Cariaco Basin of marine Mo and $\mathrm{Cd}(1)$ as particulate organic matter settling out of the photic zone (the biogenic fraction) and (2) from bottom water under conditions of $\mathrm{SO}_{4}^{2-}$ reduction (the hydrogenous fraction). 


\title{
Trace-Element Deposition in the Cariaco Basin, Venezuela Shelf, under Sulfate-Reducing Conditions-A History of the Local Hydrography and Global Climate, 20 ka to the Present
}

\author{
By David Z. Piper and Walter E. Dean
}

\section{Abstract}

A sediment core from the Cariaco Basin on the Venezuelan continental shelf, which recovered sediment that has been dated back to $20 \mathrm{ka}$ (thousand years ago), was examined for its major-element-oxide and trace-element composition. Cadmium $(\mathrm{Cd})$, chromium $(\mathrm{Cr})$, copper $(\mathrm{Cu})$, molybdenum $(\mathrm{Mo})$, nickel (Ni), vanadium (V), and zinc $(\mathrm{Zn})$ can be partitioned between a siliciclastic, terrigenous-derived fraction and two seawater-derived fractions. The two marine fractions are (1) a biogenic fraction represented by nutrient trace elements taken up mostly in the photic zone by phytoplankton, and (2) a hydrogenous fraction that has been derived from bottom water via adsorption and precipitation reactions. This suite of trace elements contrasts with a second suite of trace elements-barium (Ba), cobalt (Co), gallium ( $\mathrm{Ga})$, lithium (Li), the rare-earth elements, thorium (Th), yttrium (Y), and several of the major-element oxides - that has had solely a terrigenous source. The partitioning scheme, coupled with bulk sediment accumulation rates measured by others, allows us to determine the accumulation rate of trace elements in each of the three sediment fractions and of the fractions themselves.

The current export of organic matter from the photic zone, redox conditions and advection of bottom water, and flux of terrigenous debris into the basin can be used to calculate independently trace-element depositional rates. The calculated rates show excellent agreement with the measured rates of the surface sediment. This agreement supports a model of trace-element accumulation rates in the subsurface sediment that gives a 20-kyr history of upwelling into the photic zone (that is, primary productivity), bottom-water advection and redox, and provenance. Correspondence of extrema in the geochemical signals with global changes in sea level and climate demonstrates the high degree to which the basin hydrography and provenance have responded to the paleoceanographic and paleoclimatic regimes of the last $20 \mathrm{kyr}$.

The accumulation rate of the marine fraction of Mo increased abruptly at about $14.8 \mathrm{ka}$ (calendar years), from less than $0.5 \mu \mathrm{g} \mathrm{cm}^{-2} \mathrm{yr}^{-1}$ to greater than $4 \mu \mathrm{g} \mathrm{cm}^{-2} \mathrm{yr}^{-1}$. Its accumulation rate remained high but variable until $8.6 \mathrm{ka}$, when it decreased sharply to $1 \mu \mathrm{g} \mathrm{cm}^{-2} \mathrm{yr}^{-1}$. It continued to decrease to $4.0 \mathrm{ka}$, to its lowest value for the past $15 \mathrm{kyr}$, before gradually increasing to the present. Between $14.8 \mathrm{ka}$ and $8.6 \mathrm{ka}$, its accumulation rate exhibited strong maxima at 14.4, 13.0, and $9.9 \mathrm{ka}$. The oldest maximum corresponds to melt-water pulse IA into the Gulf of Mexico. A relative minimum, centered at about $11.1 \mathrm{ka}$, corresponds to melt-water pulse IB; a strong maximum occurs in the immediately overlying sediment. The maximum at $13.0 \mathrm{ka}$ corresponds to onset of the Younger Dryas cold event. This pattern to the accumulation rate of Mo (and V) can be interpreted in terms of its deposition from bottom water of the basin, the hydrogenous fraction, under $\mathrm{SO}_{4}{ }^{2-}$-reducing conditions, during times of intense bottomwater advection $14.8 \mathrm{ka}$ to $11.1 \mathrm{ka}$ and significantly less intense bottom-water advection $11 \mathrm{ka}$ to the present.

The accumulation rate of $\mathrm{Cd}$ shows a pattern that is only slightly different from that of Mo, although its deposition was determined largely by the rain rate of organic matter into the bottom water, a biogenic fraction whose deposition was driven by upwelling of nutrient-enriched water into the photic zone. Its accumulation exhibits only moderately high rates, on average, during both melt-water pulses. Its highest rate, and that of upwelling, occurred during the Younger Dryas, and again following melt-water pulse IB. The marine fractions of $\mathrm{Cu}, \mathrm{Ni}$, and $\mathrm{Zn}$ also have a strong biogenic signal. The siliciclastic terrigenous debris, however, represents the dominant source, and host, of $\mathrm{Cu}, \mathrm{Ni}$, and $\mathrm{Zn}$. All four trace elements have a considerably weaker hydrogenous signal than biogenic signal.

Accumulation rates of the terrigenous fraction, as reflected by accumulation rates of Th and $\mathrm{Ga}$, show strong maxima at 16.2 and $12.7 \mathrm{ka}$ and minima at 14.1 and $11.1 \mathrm{ka}$. $\mathrm{Co}, \mathrm{Li}, \mathrm{REE}$, and Y have a similar distribution. The minima occurred during melt-water pulses IA and IB, the maxima during the Younger Dryas and the rise in sea level following the last glacial maximum.

\section{Introduction}

The Cariaco Basin is located on the continental shelf of Venezuela (fig. 1). It consists of two subbasins separated by 


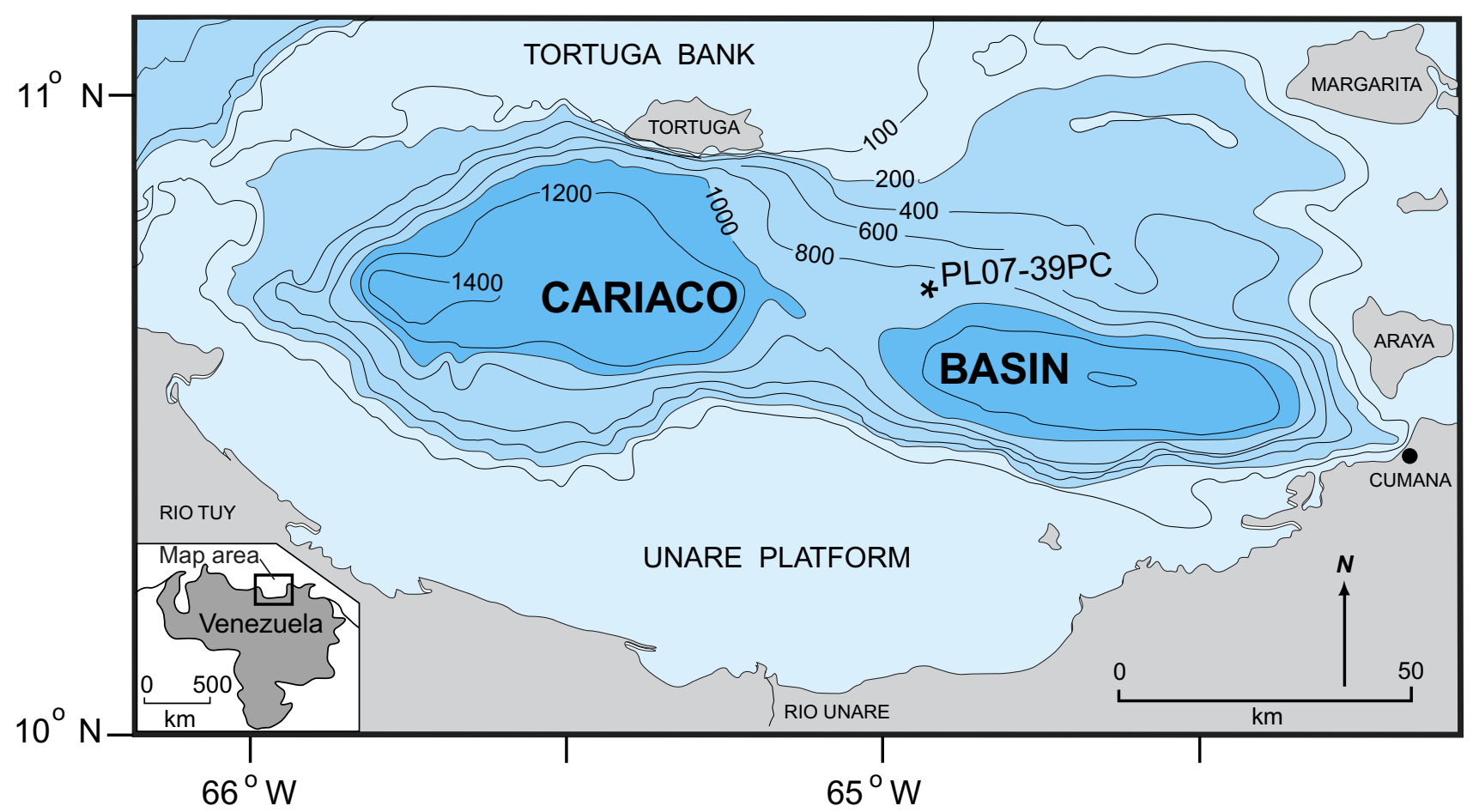

Figure 1. Bathymetry of the northwest continental shelf of Venezuela, in meters, showing location of core PL07-39PC on the ridge, or saddle, between the two subbasins. The figure is modified from Peterson and others (1995).

an 900-m deep saddle. The basin is more than $1400 \mathrm{~m}$ deep and isolated from deep water of the Caribbean Sea by a sill that has a maximum water depth of $146 \mathrm{~m}$. The sill limits exchange of water between the Cariaco Basin and Caribbean Sea, imposing a residence time of bottom water in the basin of approximately $100 \mathrm{yr}$ (Deuser, 1973). As a result of the slow renewal of bottom water, oxidation by bacterial respiration of organic matter settling out of the photic zone has completely utilized $\mathrm{O}_{2}$ and $\mathrm{NO}_{3}^{-}$as electron acceptors and established $\mathrm{SO}_{4}{ }^{2-}$-reducing conditions below approximately $250-\mathrm{m}$ to 300-m depth (Richards and Vaccaro, 1956; Richards, 1975). Oxygen respiration occurs in the uppermost approximately $200 \mathrm{~m}$ of the water column and denitrifying conditions at the intermediate depth. This redox profile controls the removal of several trace elements from the bottom water by adsorption/ precipitation reactions (for example, $\mathrm{Cd}, \mathrm{Cu}, \mathrm{Mo}$ and $\mathrm{V}$ ) and their deposition on the sea floor (Jacobs and others, 1987; Dean and others, 1999) as a hydrogenous sediment fraction.

Organic matter and organic-matter-hosted trace elements, or trace nutrients, represent a second sediment fraction, the biogenic fraction. Their deposition is governed largely by primary productivity, which averages $1.5 \mathrm{~g} \mathrm{C} \mathrm{m}^{-2} \mathrm{~d}^{-1}$ (Varela and others, 1997). Approximately six percent of the organic matter fixed by algae in the photic zone annually is exported to depth (Müller-Karger and others, 2001), but less than two percent actually settles onto the sea floor. The four-percent difference represents the organic matter that is oxidized at depth by $\mathrm{SO}_{4}{ }^{2-}$ reduction, as it settles through the water column (Thunell and others, 2000). However, primary productivity is highly seasonal (Peterson and others, 1991). During winter and spring when the Intertropical Convergence Zone (ITCZ) is located south of the equator, strong northeast trade winds produce intense upwelling and elevated primary productivity. Remains of the plankton production are preserved in the sediment as a light-colored lamina. During summer and autumn, the ITCZ shifts north of the equator; trade winds weaken and upwelling is reduced.

The summer/autumn shift of the ITCZ produces an increase of rainfall along the northern coast of South America, resulting in an increase in the flux into the basin of a third sediment fraction, the terrigenous fraction and its hosted trace elements. The seasonally elevated accumulation of this fraction is deposited as a dark lamina that, together with the lighter-colored lamina of the winter/spring period of more intense upwelling, forms an annual couplet, or varve (Peterson and others, 1991; Hughen and others, 1996a).

During the last glacial maximum (LGM), the basin was nearly isolated from the Caribbean Sea. A rising sea level, beginning about $18 \mathrm{ka}$ (Fairbanks, 1989), imposed a complex signal of temporal changes on rates of upwelling, bottomwater advection, and the flux and composition of terrigenous debris into the basin (Peterson and others, 1991; Yarincik and others, 2000). These changes are now reflected in the lithology of the sediment. In piston cores from the deepest part of the basin (Peterson and others, 1991, 1995), the sediment is bioturbated below approximately 500 to $1,000 \mathrm{cmbsf}$ (centimeters below the sea floor). It is distinctly laminated above this unit to about $300 \mathrm{cmbsf}$. From $300 \mathrm{cmbsf}$ to the surface, the sediment continues to be laminated, but laminae are thinner and weakly defined (fig. 2). Peterson and others (1991) interpreted the lithologic change occurring between the bioturbated unit and the distinctly laminated unit, dated at about $14.8 \mathrm{ka}$ (Lin 


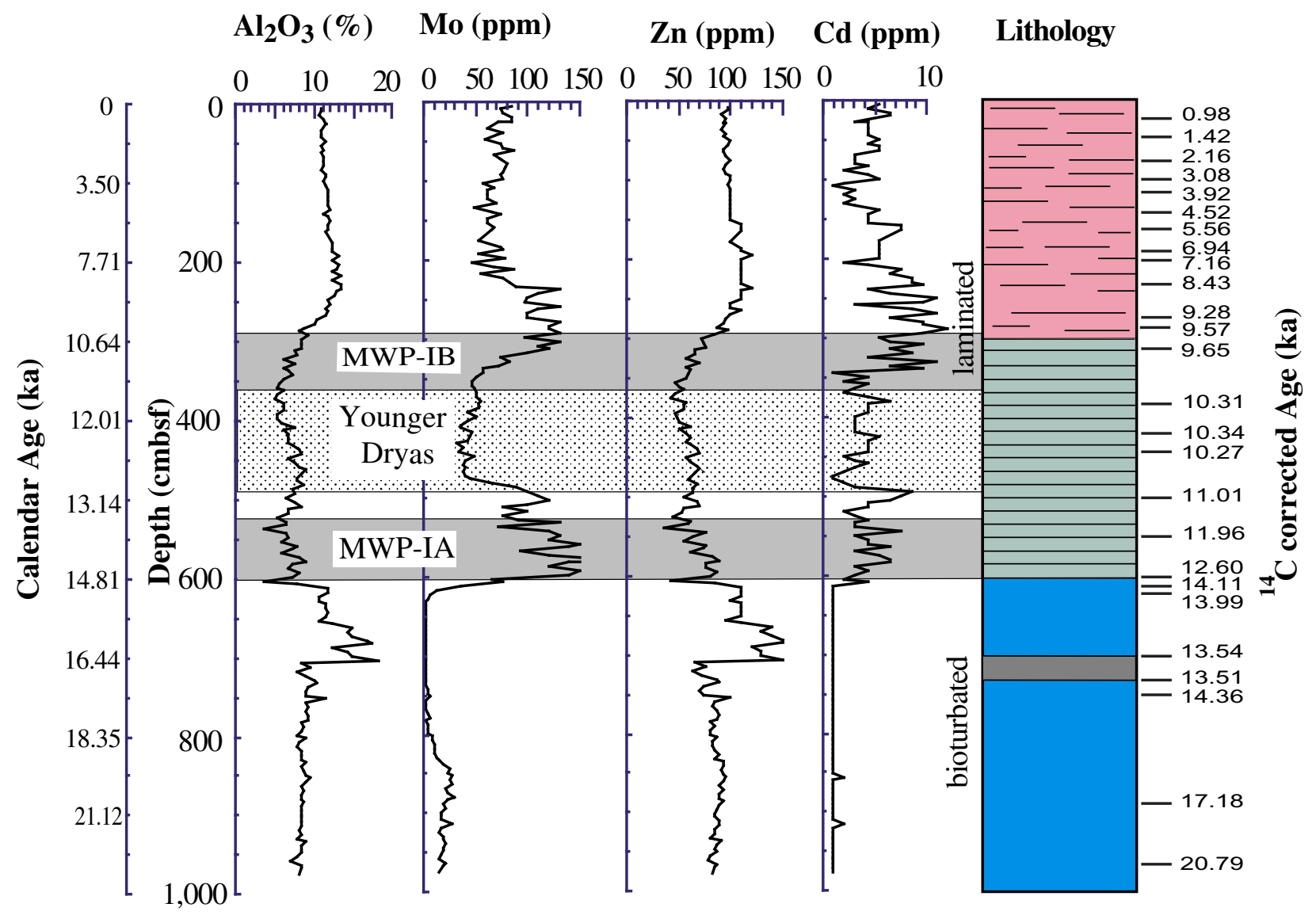

Figure 2. Concentrations of $\mathrm{Al}_{2} \mathrm{O}_{3}, \mathrm{Cd}, \mathrm{Mo}$, and $\mathrm{Zn}$ with depth in core PL07-39PC, in centimeters below the sea floor (cmbsf). Depth has been adjusted by deletion of a turbidite unit at $170 \mathrm{cmbsf}$. Sediment is faintly laminated to $300 \mathrm{cmbsf}$, distinctly laminated between 300 and $600 \mathrm{cmbsf}$, and bioturbated below $600 \mathrm{cmbsf}$, with a massive, fine-grained gray layer at $700 \mathrm{cmbsf}$ (Peterson and others, 1991; Lin and others, 1997). Radiocarbon ages are reservoir-corrected AMS ${ }^{14} \mathrm{C}$ ages as reported by Lin and others (1997). Calendar ages are given at the far left, using the age model of Hughen and others (1998). Sections of the core indicated by shading correspond to the Younger Dryas cold event and melt-water pulses IA and IB (Fairbanks, 1989).

and others, 1997; Hughen and others, 1998), as recording the onset of elevated primary productivity in the photic zone and of $\mathrm{SO}_{4}^{2-}$-reducing conditions in the bottom water. Such reducing conditions have continued to the present (Richards, 1975).

The accumulation rates of trace elements should have recorded these changes in the hydrography, primary productivity, and redox of the water column. As each source of elements-terrigenous, marine-biogenic, and marine-hydrogenous-contributes a unique inventory of several elements, partitioning of major-element oxides and trace elements into the three fractions provides a check of the calculations of the depositional rate of each fraction and the interpretation of the dynamics of the environment that controlled that rate. Trace elements in the terrigenous fraction should have been controlled merely by the flux of terrigenous siliciclastic debris into the basin, but ultimately by provenance. The rate of accumulation of the trace-element inventory in excess of the terrigenous contribution should have been governed by (1) the export of organic-matter-bound trace elements, or trace nutrients, out of the photic zone and onto the sea floor and (2) redox conditions in the bottom water. The flux of organic matter settling into the bottom water contributed to both of these factors. Organic matter carries trace nutrients directly to the sea floor, for example, $\mathrm{Cd}, \mathrm{Cu}, \mathrm{Ni}$, and $\mathrm{Zn}$, and it is the dominant reductant whose oxidation establishes the level of redox conditions $-\mathrm{O}_{2}$ respiration, denitrification, or $\mathrm{SO}_{4}{ }^{2-}$ reduction-in the water column. The flux, into the bottom water, of the three oxidants- $-\mathrm{O}_{2}, \mathrm{NO}_{3}{ }^{-}$, and $\mathrm{SO}_{4}{ }^{2-}-$ and of trace elements that make up the hydrogenous fraction can be expressed in terms of the composition of the source water and the bottom-water residence time, or advection. Similarly, the flux of organic matter settling through the water column is determined by primary productivity, which is limited by the concentrations of nutrients in the source water and the rate of upwelling of that water into the photic zone. These relations demonstrate the primary role of the basin hydrography to the deposition of trace elements.

This study examined the distributions of several majorelement oxides and trace elements in piston core PL07-39PC (fig. 1). The current hydrography, marine chemistry, and biology of the basin have been used to develop a model of elemental depositional rates. The model rates for the trace ele- 
ments agree closely with their measured accumulation rates in the uppermost surface sediment. Reversing the problem, accumulation rates of trace elements during the last 15 to 20 $\mathrm{ka}$ are used to determine past rates of upwelling into the photic zone and bottom-water advection. Unfortunately, modeling temporal changes in the hydrography, biology, and chemistry of the basin from the accumulation rates of trace elements is unavoidably limited by possible changes in the trace-element composition of terrigenous debris, plankton (biogenic debris), and source seawater from their current composition. On the plus side, the occurrence of laminated sediment in the uppermost approximately $600 \mathrm{~cm}$ of core PL07-39PC (fig. 2) suggests that the trace-element record for the most recent $14.8 \mathrm{kyr}$ has not been made more complex by biological and physical reworking of the sediment.

Acknowledgments-Comments and suggestions by colleagues at the U.S. Geological Survey-George Breit, Randolph Koski, Victor Mossotti, and Michele Tuttle_-are greatly appreciated.

\section{Analytical Techniques}

Sediment samples, 1-cm thick, were taken from piston core PL07-39PC at a 5-cm interval (table 1). Samples were dried at $90^{\circ} \mathrm{C}$, then ground to pass a 100 -mesh sieve. Elemental concentrations were determined by inductively coupled, argon-plasma, emission spectrometry (ICP-AES) in the analytical laboratories of the U.S. Geological Survey (USGS) (Baedecker, 1987). Concentrations of major-element oxides and trace elements in most samples exceed their detection limit by more than tenfold, which allows for a precision of less than 5 per cent. $\mathrm{Cd}$ is the sole major exception, for which most samples from the laminated section of the core have a concentration in the range of twofold to fivefold the detection limit, which gives a precision of approximately 10 percent. The precision has been established by comparing analyses of selected samples analyzed by ICP-AES, neutron activation analysis, and X-ray fluorescence, carried out in three different laboratories (Piper and Isaacs, 1994, 1996). Additional discussions of the analytical techniques, precision, and accuracy are given in Baedecker (1987).

\section{The Age Model}

The age model for the core, developed by Hughen and others (1998), plus dry bulk density measured every $5 \mathrm{~cm}$ permit calculations of bulk sediment and component accumulation rates. Their age model (fig. 3) is based on 29 age determinations, by AMS ${ }^{14} \mathrm{C}$, of hand picked samples of the planktonic foraminifera Globigerina bulloides (Lin and others, 1997). Ages were corrected (fig. 2) for a 420 -year marine- $-{ }^{14} \mathrm{C}$ reservoir. Hughen and others (1998) used varve chronology to calibrate the corrected radiocarbon ages between
12.6 and $7.6{ }^{14} \mathrm{C}$ kyr, tree rings (Komer and Becker, 1993) to calibrate radiocarbon ages after $7.6{ }^{14} \mathrm{C} \mathrm{kyr}$, and correlation of gray-scale measurements in cores from this basin with accumulation rates in the GISP2 Greenland ice core to calibrate radiocarbon ages greater than $13{ }^{14} \mathrm{C}$ kyr. Lin and others (1997) further subtracted out a turbidite unit that occurs between 170 and 200 cmbsf. The corrected ${ }^{14} \mathrm{C}$ ages, as reported by Lin and others (1997), are given in figure 2. Throughout the rest of the paper, ages are given in calendar years B.P. and designated as ka (kiloannum).

\section{Partitioning of Elements Between Terrigenous- and Seawater-Source Fractions-a Trace-Element Model}

Identifying the hydrography, marine chemistry, and biology of deposition of a sedimentary unit from its marine fraction of trace elements begins by determining the terrigenous contribution in each sample. The terrigenous trace-element contribution is subtracted from the bulk trace-element composition to give the marine, or seawater, contribution. The concentration of $\mathrm{Al}_{2} \mathrm{O}_{3}$ is commonly used as a measure of the concentration of the terrigenous fraction (Leinen, 1977; Isaacs, 1980; Dymond, 1981; Medrano and Piper, 1995). For the Cariaco Basin, the $\mathrm{Al}_{2} \mathrm{O}_{3}$ concentration in the terrigenous fraction itself is determined from the relation between $\mathrm{Al}_{2} \mathrm{O}_{3}$ and $\mathrm{CaO}$. The highest values in a plot of the two element oxides (fig. 4) represent a mixing curve for calcite and siliciclastic debris. Calcite is derived largely from the water column (Werne and others, 2000) and the siliciclastic debris is ter-

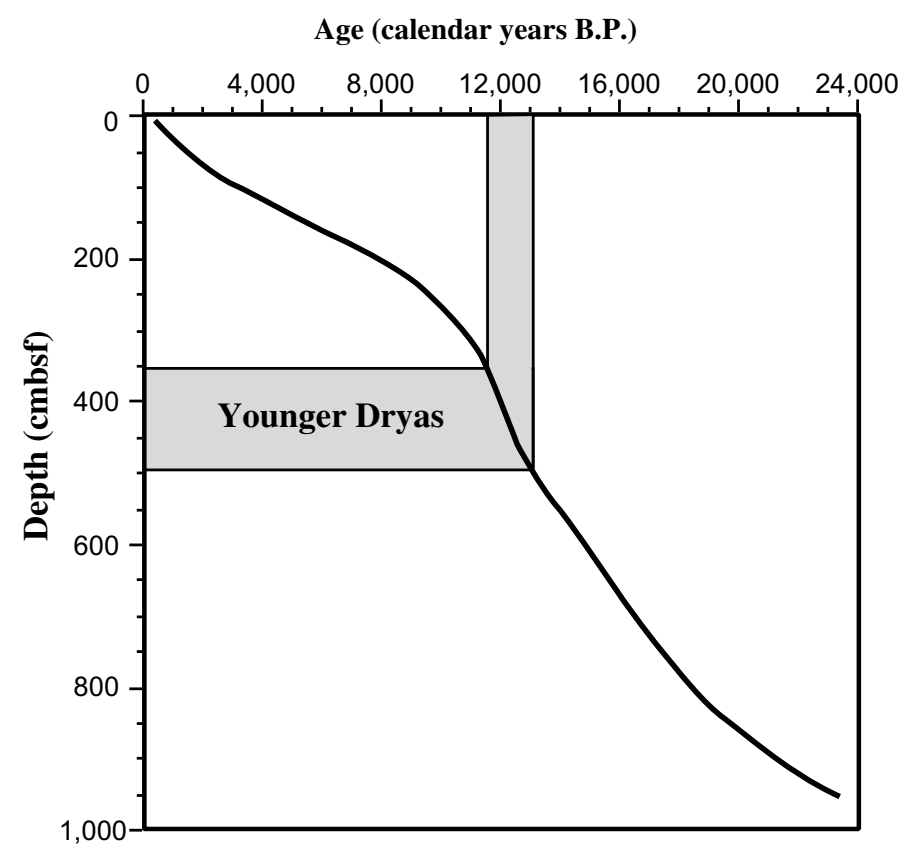

Figure 3. Age model for Cariaco Basin sediment taken from Hughen and others (1998). Sediment depth is in centimeters below the sea floor (cbsf). 
Table 1. Concentrations in sediment of core PL07-39PC of major-element oxides.

[All concentrations in percent, except for $\mathrm{MnO}$ and trace elements, which are in parts per million. Elemental analyses are by inductively coupled, argonplasma, emission spectrometry (ICP-AES). Ages are in calendar years B.P.]

\begin{tabular}{|c|c|c|c|c|c|c|c|c|c|c|c|}
\hline Sample & 39PC004 & 39PC008 & 39PC013 & 39PC018 & $39 \mathrm{PC} 024$ & $39 \mathrm{PC} 025$ & 39PC034 & 39PC038 & 39PC042 & 39PC047 & 39PC052 \\
\hline Depth (cm)-- & 4 & 8.5 & 13 & 18 & 24 & 26 & 34 & 38 & 42 & 47 & 52 \\
\hline Age (yr)----- & 464 & 576 & 687 & 795 & 901 & 1010 & 1121 & 1243 & 1366 & 1505 & 1647 \\
\hline $\mathrm{Al}_{2} \mathrm{O}_{3}$ & 10.96 & 10.96 & 10.77 & 11.14 & 11.33 & 11.33 & 10.96 & 10.96 & 10.96 & 11.52 & 10.96 \\
\hline CaO------- & 14 & 14 & 15.4 & 14 & 13.86 & 14 & 15.4 & 15.4 & 15.4 & 15.4 & 15.4 \\
\hline MgO--------. & 2.16 & 2.16 & 1.99 & 2.16 & 2.16 & 2.16 & 1.99 & 1.99 & 1.99 & 2.16 & 1.99 \\
\hline $\mathrm{TiO}_{2}$ & 0.35 & 0.35 & 0.33 & 0.33 & 0.33 & 0.37 & 0.33 & 0.35 & 0.35 & 0.35 & 0.33 \\
\hline $\mathrm{K}_{2} \mathrm{O}$ & 1.57 & 1.57 & 1.45 & 1.57 & 1.57 & 1.57 & 1.45 & 1.45 & 1.45 & 1.57 & 1.57 \\
\hline $\mathrm{Fe}_{2} \mathrm{O}_{3}$ & 3.72 & 3.72 & 3.57 & 3.86 & 3.72 & 3.86 & 3.72 & 3.72 & 3.72 & 3.86 & 4 \\
\hline $\mathrm{P}_{2} \mathrm{O}_{5}$--------- & 0.32 & 0.21 & 0.25 & 0.23 & 0.23 & 0.23 & 0.23 & 0.23 & 0.23 & 0.23 & 0.27 \\
\hline MnO-------. & 194 & 181 & 194 & 220 & 181 & 194 & 194 & 194 & 181 & 207 & 181 \\
\hline As-- & 7 & 7 & 7 & 7 & 7 & 7 & 7 & 7 & 7 & 10 & 7 \\
\hline Ba----------. & 240 & 250 & 250 & 260 & 260 & 260 & 250 & 260 & 260 & 240 & 260 \\
\hline Cd-------- & 5 & 4 & 6 & 6 & 3 & 4 & 4 & 4 & 4 & 5 & 4 \\
\hline Ce---------- & 36 & 34 & 33 & 35 & 36 & 37 & 32 & 34 & 36 & 36 & 34 \\
\hline Co----------- & 11 & 9 & 9 & 9 & 9 & 10 & 9 & 11 & 9 & 10 & 9 \\
\hline Cr------------ & 100 & 100 & 97 & 110 & 110 & 100 & 100 & 100 & 100 & 110 & 100 \\
\hline Cu-------- & 26 & 26 & 26 & 26 & 26 & 26 & 23 & 25 & 27 & 26 & 25 \\
\hline Ga----------- & 12 & 13 & 12 & 13 & 12 & 13 & 12 & 12 & 12 & 14 & 13 \\
\hline La-----------. & 22 & 22 & 21 & 22 & 22 & 23 & 21 & 22 & 22 & 23 & 22 \\
\hline Li----------- & 53 & 52 & 51 & 53 & 54 & 55 & 53 & 53 & 52 & 55 & 54 \\
\hline Mo---------- & 84 & 74 & 76 & 84 & 84 & 71 & 62 & 75 & 70 & 59 & 74 \\
\hline Nb----------- & 6 & 7 & 6 & 6 & 6 & 7 & 6 & 5 & 5 & 5 & 6 \\
\hline Nd------------ & 20 & 21 & 20 & 20 & 19 & 22 & 20 & 19 & 21 & 22 & 18 \\
\hline Ni--------- & 63 & 59 & 64 & 66 & 57 & 60 & 59 & 61 & 61 & 62 & 62 \\
\hline Pb---------- & 11 & 12 & 11 & 10 & 9 & 10 & 9 & 11 & 10 & 10 & 11 \\
\hline Sr----------- & 600 & 590 & 610 & 580 & 600 & 610 & 640 & 630 & 630 & 630 & 660 \\
\hline Th-----------. & 7 & 7 & 6 & 7 & 7 & 8 & 6 & 6 & 7 & 6 & 7 \\
\hline 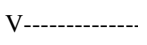 & 190 & 180 & 200 & 190 & 180 & 180 & 180 & 200 & 190 & 190 & 200 \\
\hline Y---------- & 9 & 9 & 9 & 9 & 9 & 10 & 9 & 9 & 9 & 9 & 9 \\
\hline Zn----------. & 97 & 98 & 92 & 96 & 93 & 96 & 92 & 95 & 94 & 99 & 98 \\
\hline Sample & 39PC057 & 39PC062 & 39PC067 & 39PC072 & 39PC077 & 39PC082 & 39PC087 & 39PC092 & 39PC097 & $39 \mathrm{PC} 102$ & 39PC107 \\
\hline Depth (cm)-- & 57 & 62 & 67 & 72 & 77 & 82 & 87 & 92 & 97 & 102 & 107 \\
\hline Age (yr)----- & 1816 & 1987 & 2167 & 2349 & 2539 & 2735 & 2942 & 3151 & 3363 & 3587 & 3823 \\
\hline $\mathrm{Al}_{2} \mathrm{O}_{3}$ & 11.14 & 10.96 & 11.14 & 11.14 & 11.14 & 10.96 & 11.33 & 11.52 & 11.14 & 11.52 & 11.71 \\
\hline $\mathrm{CaO}-$ & 15.4 & 15.4 & 16.8 & 15.4 & 15.4 & 15.4 & 16.8 & 15.4 & 16.8 & 16.8 & 15.4 \\
\hline MgO-------- & 1.99 & 1.99 & 1.99 & 1.99 & 1.99 & 1.99 & 1.99 & 1.99 & 1.99 & 1.99 & 1.99 \\
\hline $\mathrm{TiO}_{2}$ & 0.33 & 0.35 & 0.37 & 0.35 & 0.35 & 0.35 & 0.37 & 0.4 & 0.37 & 0.38 & 0.38 \\
\hline $\mathrm{K}_{2} \mathrm{O}$ & 1.45 & 1.57 & 1.57 & 1.57 & 1.57 & 1.45 & 1.57 & 1.57 & 1.45 & 1.57 & 1.57 \\
\hline $\mathrm{Fe}_{2} \mathrm{O}_{3}$ & 3.72 & 3.72 & 3.72 & 4 & 3.86 & 3.86 & 4 & 4 & 3.86 & 3.86 & 4.15 \\
\hline $\mathrm{P}_{2} \mathrm{O}_{5}$ & 0.27 & 0.23 & 0.25 & 0.39 & 0.23 & 0.25 & 0.25 & 0.39 & 0.21 & 0.25 & 0.23 \\
\hline MnO------- & 194 & 181 & 181 & 181 & 168 & 181 & 181 & 207 & 168 & 181 & 207 \\
\hline As------------ & 7 & 7 & 7 & 7 & 7 & 7 & 7 & 10 & 7 & 7 & 12 \\
\hline Ba---------- & 260 & 280 & 270 & 270 & 280 & 280 & 290 & 280 & 280 & 240 & 300 \\
\hline Cd----------- & 5 & 5 & 3 & 3 & 3 & 4 & 2 & 4 & 5 & 2 & 1 \\
\hline Ce------- & 35 & 34 & 36 & 35 & 35 & 35 & 37 & 38 & 35 & 38 & 39 \\
\hline Co----------- & 10 & 10 & 9 & 10 & 9 & 9 & 9 & 10 & 9 & 10 & 9 \\
\hline Cr---------. & 100 & 100 & 100 & 110 & 100 & 110 & 110 & 110 & 100 & 110 & 110 \\
\hline Cu------- & 27 & 26 & 25 & 25 & 25 & 27 & 25 & 24 & 25 & 24 & 24 \\
\hline Ga----------- & 13 & 12 & 13 & 13 & 12 & 12 & 13 & 12 & 13 & 13 & 13 \\
\hline La---------- & 22 & 22 & 22 & 22 & 22 & 22 & 23 & 24 & 22 & 24 & 23 \\
\hline Li---------- & 53 & 54 & 54 & 53 & 54 & 53 & 55 & 56 & 54 & 56 & 56 \\
\hline Mo--------- & 75 & 86 & 68 & 73 & 81 & 78 & 75 & 74 & 77 & 58 & 68 \\
\hline Nb----------- & 5 & 6 & 7 & 6 & 6 & 6 & 6 & 7 & 7 & 7 & 6 \\
\hline Nd------------ & 19 & 19 & 21 & 20 & 21 & 19 & 21 & 21 & 20 & 21 & 20 \\
\hline Ni--------- & 63 & 63 & 62 & 61 & 61 & 63 & 61 & 63 & 64 & 62 & 59 \\
\hline Pb-------- & 9 & 10 & 9 & 9 & 10 & 8 & 10 & 10 & 13 & 12 & 10 \\
\hline Sr----------- & 650 & 660 & 660 & 650 & 660 & 660 & 670 & 690 & 680 & 660 & 680 \\
\hline Th---------- & 7 & 6 & 7 & 6 & 7 & 6 & 7 & 6 & 7 & 8 & 7 \\
\hline 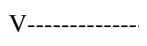 & 200 & 200 & 190 & 180 & 190 & 190 & 180 & 180 & 200 & 180 & 180 \\
\hline Y--------- & 9 & 9 & 9 & 10 & 9 & 9 & 9 & 10 & 9 & 10 & 10 \\
\hline Zn----------- & 94 & 94 & 96 & 100 & 95 & 94 & 96 & 100 & 100 & 98 & 100 \\
\hline
\end{tabular}


Table 1. Concentrations in sediment of core PL07-39PC of major-element oxides-Continued.

\begin{tabular}{|c|c|c|c|c|c|c|c|c|c|c|c|}
\hline Sample & $39 \mathrm{PC} 112$ & $39 \mathrm{PC} 117$ & 39PC122 & 39PC127 & 39PC132 & 39PC137 & 39PC142 & $39 \mathrm{PC} 147$ & 39PC152 & 39PC157 & 39PC162 \\
\hline Depth (cm)-- & 112 & 117 & 122 & 127 & 132 & 137 & 142 & 147 & 152 & 157 & 162 \\
\hline Age (yr)----. & 4062 & 4303 & 4532 & 4756 & 4981 & 5207 & 5439 & 5671 & 5911 & 6151 & 6397 \\
\hline $\mathrm{Al}_{2} \mathrm{O}_{3}$ & 11.9 & 11.9 & 11.71 & 11.71 & 12.09 & 11.14 & 11.71 & 12.09 & 11.71 & 11.9 & 11.52 \\
\hline 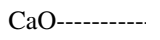 & 15.4 & 16.8 & 16.8 & 15.4 & 15.4 & 18.2 & 16.8 & 15.4 & 16.8 & 16.8 & 16.8 \\
\hline MgO-------. & 1.99 & 1.99 & 1.99 & 2.16 & 2.16 & 1.99 & 2.16 & 1.99 & 1.99 & 1.99 & 1.99 \\
\hline $\mathrm{TiO}_{2}$ & 0.38 & 0.33 & 0.38 & 0.17 & 0.37 & 0.37 & 0.37 & 0.37 & 0.38 & 0.37 & 0.37 \\
\hline $\mathrm{K}_{2} \mathrm{O}-$ & 1.57 & 1.57 & 1.57 & 1.57 & 1.57 & 1.45 & 1.57 & 1.57 & 1.57 & 1.69 & 1.57 \\
\hline $\mathrm{Fe}_{2} \mathrm{O}_{3}$ & 4.29 & 4.29 & 4 & 4.15 & 4.15 & 3.86 & 4.15 & 4.15 & 4.15 & 4.15 & 4 \\
\hline $\mathrm{P}_{2} \mathrm{O}_{5}$--------- & 0.23 & 0.23 & 0.25 & 0.34 & 0.23 & 0.23 & 0.27 & 0.23 & 0.3 & 0.23 & 0.3 \\
\hline MnO------- & 232 & 207 & 207 & 181 & 232 & 232 & 207 & 220 & 194 & 271 & 220 \\
\hline As----------- & 11 & 11 & 7 & 7 & 7 & 7 & 7 & 7 & 7 & 7 & 7 \\
\hline Ba---------. & 270 & 280 & 300 & 300 & 280 & 310 & 330 & 330 & 370 & 380 & 400 \\
\hline Cd-- & 3 & 2 & 3 & 2 & 3 & 5 & 4 & 4 & 4 & 7 & 7 \\
\hline 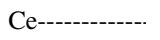 & 37 & 33 & 37 & 22 & 37 & 35 & 36 & 37 & 38 & 36 & 36 \\
\hline Co---------. & 10 & 11 & 10 & 10 & 11 & 10 & 10 & 11 & 10 & 11 & 10 \\
\hline Cr---------- & 120 & 110 & 110 & 110 & 120 & 110 & 110 & 120 & 120 & 120 & 120 \\
\hline Cu--------.. & 26 & 25 & 25 & 25 & 25 & 26 & 25 & 26 & 26 & 24 & 28 \\
\hline Ga--------- & 14 & 13 & 14 & 13 & 13 & 13 & 13 & 14 & 14 & 13 & 14 \\
\hline La--------- & 24 & 21 & 23 & 14 & 24 & 22 & 23 & 23 & 24 & 23 & 23 \\
\hline Li---------- & 57 & 58 & 57 & 55 & 58 & 53 & 55 & 58 & 56 & 56 & 55 \\
\hline Mo----------- & 61 & 62 & 62 & 69 & 48 & 65 & 73 & 62 & 62 & 68 & 66 \\
\hline Nb---------- & 6 & 5 & 6 & 3 & 7 & 5 & 6 & 6 & 6 & 5 & 5 \\
\hline Nd---------- & 21 & 21 & 22 & 16 & 21 & 22 & 20 & 20 & 23 & 22 & 20 \\
\hline Ni----------- & 65 & 62 & 64 & 63 & 68 & 71 & 67 & 69 & 66 & 76 & 72 \\
\hline Pb----------- & 11 & 10 & 10 & 11 & 10 & 11 & 11 & 12 & 14 & 12 & 14 \\
\hline Sr---------- & 670 & 710 & 690 & 680 & 670 & 730 & 710 & 640 & 690 & 690 & 710 \\
\hline Th--------. & 7 & 6 & 7 & 6 & 6 & 7 & 6 & 6 & 7 & 7 & 7 \\
\hline V-----------. & 190 & 170 & 190 & 180 & 180 & 180 & 170 & 190 & 180 & 190 & 180 \\
\hline Y-------- & 10 & 9 & 10 & 8 & 10 & 9 & 10 & 10 & 10 & 10 & 10 \\
\hline Zn----------. & 100 & 100 & 100 & 100 & 100 & 100 & 100 & 100 & 110 & 110 & 110 \\
\hline Sample & 39PC202 & 39PC207 & 39PC212 & 39PC217 & 39PC222 & $39 \mathrm{PC} 227$ & 39PC232 & 39PC237 & 39PC242 & 39PC247 & $39 \mathrm{PC} 252$ \\
\hline Depth (cm)-- & 182 & 187 & 192 & 197 & 202 & 207 & 212 & 217 & 222 & 227 & 232 \\
\hline Age (yr)------ & 6898 & 7146 & 7370 & 7586 & 7787 & 7987 & 8188 & 8385 & 8577 & 8756 & 8924 \\
\hline $\mathrm{Al}_{2} \mathrm{O}_{3}$ & 12.28 & 12.47 & 13.03 & 12.47 & 13.03 & 12.84 & 12.28 & 13.6 & 12.47 & 13.6 & 13.6 \\
\hline CaO-------. & 15.4 & 15.4 & 13.44 & 15.4 & 14 & 13.86 & 14 & 12.6 & 13.44 & 11.62 & 11.2 \\
\hline MgO-------- & 1.99 & 1.99 & 1.99 & 2.16 & 2.16 & 2.16 & 2.16 & 2.16 & 1.99 & 1.99 & 1.99 \\
\hline $\mathrm{TiO}_{2}$ & 0.38 & 0.38 & 0.42 & 0.38 & 0.42 & 0.4 & 0.38 & 0.43 & 0.42 & 0.45 & 0.4 \\
\hline $\mathrm{K}_{2} \mathrm{O}$ & 1.69 & 1.69 & 1.81 & 1.81 & 1.81 & 1.81 & 1.69 & 1.93 & 1.81 & 1.93 & 1.93 \\
\hline $\mathrm{Fe}_{2} \mathrm{O}_{3}$ & 4.43 & 4.43 & 4.58 & 4.43 & 4.58 & 4.58 & 4.43 & 4.72 & 4.58 & 4.72 & 5 \\
\hline $\mathrm{P}_{2} \mathrm{O}_{5}$ & 0.23 & 0.23 & 0.23 & 0.21 & 0.23 & 0.23 & 0.25 & 0.21 & 0.32 & 0.21 & 0.16 \\
\hline MnO-------- & 220 & 245 & 271 & 220 & 220 & 232 & 207 & 245 & 220 & 220 & 232 \\
\hline As----------- & 11 & 11 & 10 & 11 & 11 & 7 & 11 & 7 & 7 & 7 & 10 \\
\hline Ba---------- & 470 & 510 & 340 & 740 & 290 & 300 & 290 & 300 & 290 & 310 & 280 \\
\hline Cd---------. & 5 & 5 & 5 & 5 & 2 & 4 & 7 & 6 & 8 & 8 & 9 \\
\hline Ce------- & 44 & 45 & 47 & 46 & 48 & 46 & 44 & 50 & 49 & 54 & 48 \\
\hline Co--------. & 12 & 11 & 12 & 11 & 11 & 13 & 12 & 11 & 12 & 13 & 15 \\
\hline Cr--------- & 120 & 130 & 140 & 140 & 150 & 150 & 130 & 140 & 120 & 130 & 110 \\
\hline Cu---------. & 26 & 26 & 27 & 27 & 26 & 27 & 28 & 25 & 27 & 24 & 26 \\
\hline Ga----------. & 16 & 14 & 16 & 15 & 15 & 16 & 15 & 16 & 16 & 17 & 16 \\
\hline La---------- & 28 & 28 & 30 & 28 & 31 & 28 & 28 & 31 & 30 & 32 & 30 \\
\hline Li------ & 58 & 58 & 61 & 59 & 61 & 60 & 57 & 64 & 59 & 63 & 63 \\
\hline Mo----------- & 73 & 75 & 54 & 79 & 46 & 68 & 88 & 56 & 76 & 82 & 90 \\
\hline Nb---------י & 6 & 6 & 7 & 5 & 7 & 6 & 6 & 8 & 6 & 6 & 6 \\
\hline Nd----------- & 23 & 22 & 24 & 23 & 25 & 24 & 23 & 26 & 24 & 27 & 24 \\
\hline Ni----------- & 72 & 81 & 86 & 88 & 76 & 91 & 85 & 75 & 76 & 78 & 88 \\
\hline Pb----------- & 8 & 11 & 11 & 9 & 11 & 8 & 11 & 11 & 9 & 10 & 11 \\
\hline Sr-----------.. & 640 & 680 & 600 & 670 & 600 & 600 & 620 & 590 & 590 & 530 & 510 \\
\hline Th------------ & 9 & 8 & 10 & 9 & 10 & 8 & 9 & 9 & 9 & 10 & 10 \\
\hline V---------- & 170 & 180 & 190 & 190 & 160 & 180 & 190 & 180 & 190 & 190 & 200 \\
\hline Y----------- & 11 & 11 & 12 & 11 & 12 & 12 & 12 & 13 & 12 & 13 & 12 \\
\hline Zn--------- & 110 & 110 & 120 & 110 & 110 & 110 & 110 & 110 & 110 & 110 & 120 \\
\hline
\end{tabular}


Table 1. Concentrations in sediment of core PL07-39PC of major-element oxides-Continued.

\begin{tabular}{|c|c|c|c|c|c|c|c|c|c|c|c|}
\hline Sample & 39PC257 & 39 PC262 & $39 P C 267$ & $39 P C 272$ & 39PC277 & 39PC282 & $39 \mathrm{PC} 287$ & 39PC292 & 39PC297 & 39PC302 & 39 PC 307 \\
\hline Depth (cm)-- & 237 & 242 & 247 & 252 & 257 & 262 & 267 & 272 & 277 & 282 & 287 \\
\hline Age (yr)----- & 9080 & 9229 & 9369 & 9506 & 9635 & 9764 & 9888 & 10011 & 10129 & 10247 & 10362 \\
\hline $\mathrm{Al}_{2} \mathrm{O}_{3}$ & 12.84 & 12.66 & 11.9 & 12.09 & 11.52 & 11.71 & 11.52 & 10.39 & 10.2 & 8.69 & 8.12 \\
\hline $\mathrm{CaO}-$ & 11.2 & 11.48 & 13.02 & 13.02 & 15.4 & 12.74 & 13.3 & 13.72 & 11.76 & 11.76 & 11.9 \\
\hline MgO---------- & 1.99 & 1.99 & 1.82 & 1.82 & 1.82 & 1.82 & 1.82 & 1.99 & 1.82 & 1.82 & 1.82 \\
\hline $\mathrm{TiO}_{2}$ & 0.42 & 0.4 & 0.4 & 0.38 & 0.4 & 0.38 & 0.37 & 0.35 & 0.33 & 0.28 & 0.27 \\
\hline $\mathrm{K}_{2} \mathrm{O}-$---------- & 1.81 & 1.81 & 1.69 & 1.69 & 1.57 & 1.57 & 1.57 & 1.45 & 1.45 & 1.33 & 1.2 \\
\hline $\mathrm{Fe}_{2} \mathrm{O}_{3}$ & 4.72 & 4.58 & 4.29 & 4.29 & 4.15 & 4.29 & 4.15 & 3.72 & 3.72 & 3.15 & 3 \\
\hline $\mathrm{P}_{2} \mathrm{O}_{5}$ & 0.23 & 0.21 & 0.18 & 0.21 & 0.21 & 0.23 & 0.21 & 0.27 & 0.21 & 0.53 & 0.6 \\
\hline MnO---------- & 194 & 207 & 207 & 207 & 181 & 181 & 194 & 155 & 155 & 129 & 120 \\
\hline As------------- & 7 & 10 & 7 & 7 & 7 & 7 & 7 & 7 & 7 & 7 & 7 \\
\hline Ва-------------. & 320 & 260 & 250 & 250 & 280 & 260 & 260 & 290 & 220 & 210 & 210 \\
\hline Cd--------. & 4 & 6 & 10 & 9 & 3 & 8 & 10 & 6 & 9 & 9 & 11 \\
\hline Ce------------. & 51 & 50 & 47 & 47 & 48 & 46 & 47 & 43 & 42 & 37 & 35 \\
\hline 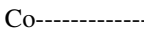 & 11 & 12 & 12 & 11 & 10 & 10 & 11 & 9 & 9 & 8 & 8 \\
\hline Cr------------- & 100 & 110 & 110 & 100 & 100 & 100 & 100 & 100 & 98 & 89 & 84 \\
\hline Cu--------.. & 25 & 26 & 27 & 26 & 25 & 25 & 25 & 24 & 26 & 22 & 22 \\
\hline Ga-----------.. & 15 & 15 & 14 & 14 & 14 & 14 & 14 & 12 & 13 & 10 & 10 \\
\hline La------------ & 31 & 30 & 30 & 29 & 29 & 29 & 28 & 27 & 26 & 22 & 22 \\
\hline Li------------.' & 61 & 59 & 56 & 57 & 55 & 55 & 54 & 49 & 47 & 41 & 38 \\
\hline Mo------------ & 130 & 110 & 100 & 97 & 130 & 110 & 100 & 100 & 130 & 120 & 120 \\
\hline Nb-----------. & 7 & 7 & 7 & 6 & 7 & 7 & 6 & 6 & 5 & 6 & 5 \\
\hline Nd----------.' & 24 & 24 & 24 & 24 & 24 & 25 & 24 & 22 & 22 & 18 & 16 \\
\hline Ni------------ & 64 & 72 & 82 & 68 & 62 & 68 & 68 & 62 & 61 & 57 & 54 \\
\hline Pb------------- & 11 & 11 & 11 & 10 & 8 & 11 & 12 & 8 & 11 & 9 & 9 \\
\hline Sr----------- & 510 & 510 & 570 & 560 & 610 & 540 & 580 & 610 & 500 & 550 & 550 \\
\hline Th------------ & 10 & 8 & 9 & 9 & 9 & 9 & 8 & 7 & 8 & 7 & 6 \\
\hline V------------ & 200 & 210 & 170 & 170 & 170 & 180 & 180 & 160 & 170 & 170 & 160 \\
\hline Y------------ & 13 & 12 & 12 & 12 & 12 & 12 & 13 & 12 & 11 & 10 & 10 \\
\hline Zn-----.- & 110 & 110 & 110 & 100 & 100 & 110 & 100 & 96 & 93 & 87 & 97 \\
\hline Sample & 39PC311 & 39PC317 & $39 \mathrm{PC} 322$ & 39PC327 & 39PC332 & 39PC338 & 39PC343 & 39PC347 & 39PC353 & 39PC357 & 39PC362 \\
\hline Depth $(\mathrm{cm})--$ & 291 & 297 & 302 & 307 & 312 & 318 & 323 & 327 & 333 & 337 & 342 \\
\hline Age (yr)----. & 10478 & 10591 & 10703 & 10808 & 10907 & 10994 & 11077 & 11155 & 11229 & 11300 & 11367 \\
\hline $\mathrm{Al}_{2} \mathrm{O}_{3}$ & 9.26 & 8.31 & 8.31 & 8.31 & 7.56 & 7.74 & 6.23 & 7.56 & 6.04 & 6.8 & 7.18 \\
\hline CaO--------- & 11.48 & 11.2 & 10.92 & 11.06 & 9.94 & 11.06 & 9.52 & 11.34 & 11.62 & 16.8 & 19.6 \\
\hline MgO--------. & 1.82 & 1.82 & 1.82 & 1.82 & 1.64 & 1.66 & 1.66 & 1.82 & 1.82 & 2.32 & 2.32 \\
\hline $\mathrm{TiO}_{2}$ & 0.3 & 0.23 & 0.25 & 0.27 & 0.25 & 0.25 & 0.2 & 0.25 & 0.2 & 0.23 & 0.25 \\
\hline $\mathrm{K}_{2} \mathrm{O}$ & 1.33 & 1.33 & 1.2 & 1.2 & 1.13 & 1.16 & 1.02 & 1.17 & 0.98 & 1.04 & 1.06 \\
\hline $\mathrm{Fe}_{2} \mathrm{O}_{3}$ & 3.43 & 3 & 3 & 3 & 2.72 & 2.72 & 2.29 & 2.86 & 2.29 & 2.57 & 2.57 \\
\hline $\mathrm{P}_{2} \mathrm{O}_{5}$ & 0.21 & 0.21 & 0.18 & 0.23 & 0.16 & 0.16 & 0.16 & 0.25 & 0.21 & 0.23 & 0.25 \\
\hline MnO--------. & 142 & 124 & 120 & 127 & 108 & 120 & 103 & 142 & 107 & 155 & 142 \\
\hline As-----------. & 7 & 7 & 7 & 7 & 7 & 7 & 7 & 7 & 7 & 7 & 7 \\
\hline Ba----------.. & 190 & 200 & 180 & 170 & 180 & 180 & 130 & 160 & 130 & 160 & 200 \\
\hline Cd---------- & 9 & 5 & 6 & 9 & 6 & 8 & 4 & 10 & 6 & 9 & 1 \\
\hline Ce--'---'--. & 39 & 33 & 31 & 32 & 32 & 32 & 25 & 32 & 26 & 30 & 33 \\
\hline Co----------- & 8 & 7 & 8 & 8 & 7 & 7 & 6 & 9 & 6 & 7 & 6 \\
\hline Cr------------. & 88 & 81 & 81 & 84 & 72 & 72 & 62 & 71 & 60 & 68 & 67 \\
\hline Cu---------- & 21 & 20 & 20 & 21 & 19 & 18 & 20 & 19 & 17 & 19 & 18 \\
\hline Ga------------ & 11 & 11 & 9 & 10 & 9 & 10 & 8 & 10 & 7 & 9 & 9 \\
\hline La-----------. & 24 & 20 & 20 & 21 & 19 & 20 & 16 & 19 & 16 & 19 & 20 \\
\hline Li----------- & 43 & 39 & 39 & 39 & 36 & 37 & 30 & 36 & 30 & 34 & 35 \\
\hline Mo-----------. & 130 & 98 & 130 & 110 & 120 & 100 & 73 & 82 & 72 & 58 & 58 \\
\hline Nb---------- & 5 & 5 & 5 & 5 & 4 & 5 & 3 & 4 & 3 & 4 & 5 \\
\hline Nd----------- & 19 & 16 & 14 & 16 & 16 & 17 & 13 & 16 & 12 & 17 & 17 \\
\hline Ni---------- & 52 & 46 & 54 & 53 & 44 & 46 & 33 & 50 & 35 & 50 & 38 \\
\hline Pb------------. & 10 & 8 & 8 & 7 & 8 & 7 & 6 & 7 & 6 & 5 & 7 \\
\hline Sr---------- & 530 & 520 & 520 & 520 & 450 & 530 & 460 & 550 & 560 & 870 & 960 \\
\hline Th-----------.. & 7 & 6 & 6 & 6 & 6 & 6 & 4 & 6 & 4 & 4 & 6 \\
\hline V---'-----. & 140 & 140 & 150 & 160 & 130 & 140 & 120 & 140 & 98 & 110 & 100 \\
\hline Y---------. & 10 & 9 & 9 & 9 & 9 & 9 & 7 & 9 & 8 & 9 & 10 \\
\hline 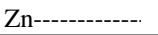 & 84 & 72 & 74 & 77 & 66 & 67 & 57 & 71 & 57 & 62 & 59 \\
\hline
\end{tabular}


Table 1. Concentrations in sediment of core PL07-39PC of major-element oxides-Continued.

\begin{tabular}{|c|c|c|c|c|c|c|c|c|c|c|c|}
\hline Sample & 39PC367 & 39PC372 & 39PC377 & 39PC382 & 39PC387 & 39PC392 & 39PC397 & 39PC402 & 39PC408 & $39 \mathrm{PC} 412$ & $39 \mathrm{PC} 417$ \\
\hline Depth (cm)-- & 347 & 352 & 357 & 362 & 367 & 372 & 377 & 382 & 387 & 392 & 397 \\
\hline Age (yr)-----. & 11433 & 11496 & 11557 & 11615 & 11673 & 11727 & 11782 & 11834 & 11885 & 11934 & 11983 \\
\hline $\mathrm{Al}_{2} \mathrm{O}_{3}$------- & 6.23 & 5.67 & 5.48 & 6.04 & 5.29 & 5.1 & 6.23 & 6.23 & 6.23 & 5.29 & 5.29 \\
\hline CaO----------. & 19.6 & 18.2 & 19.6 & 18.2 & 16.8 & 16.8 & 19.6 & 19.6 & 18.2 & 16.8 & 16.8 \\
\hline MgO--------.. & 2.32 & 2.16 & 2.16 & 2.16 & 2.16 & 2.16 & 2.16 & 2.32 & 2.16 & 2.16 & 2.16 \\
\hline $\mathrm{TiO}_{2}$---------- & 0.18 & 0.2 & 0.18 & 0.18 & 0.2 & 0.17 & 0.22 & 0.2 & 0.2 & 0.17 & 0.17 \\
\hline $\mathrm{K}_{2} \mathrm{O}$--------- & 0.95 & 0.89 & 0.84 & 0.89 & 0.83 & 0.81 & 0.93 & 0.98 & 0.95 & 0.87 & 0.86 \\
\hline $\mathrm{Fe}_{2} \mathrm{O}_{3}$-------- & 2.29 & 2 & 2 & 2.14 & 2 & 1.86 & 2.29 & 2.29 & 2.29 & 1.86 & 1.86 \\
\hline $\mathrm{P}_{2} \mathrm{O}_{5}$ & 0.25 & 0.18 & 0.21 & 0.27 & 0.18 & 0.16 & 0.18 & 0.18 & 0.18 & 0.18 & 0.18 \\
\hline MnO---------. & 142 & 115 & 112 & 119 & 103 & 101 & 128 & 112 & 114 & 94 & 90 \\
\hline As--------------. & 7 & 7 & 7 & 7 & 7 & 7 & 7 & 7 & 7 & 7 & 7 \\
\hline Ba-----------.. & 150 & 150 & 150 & 150 & 170 & 130 & 150 & 180 & 160 & 140 & 150 \\
\hline Cd------------. & 4 & 2 & 4 & 3 & 2 & 4 & 6 & 4 & 4 & 4 & 3 \\
\hline Ce-----------. & 25 & 25 & 26 & 27 & 26 & 23 & 28 & 23 & 25 & 22 & 19 \\
\hline Co------------. & 7 & 6 & 6 & 6 & 6 & 6 & 6 & 5 & 5 & 5 & 5 \\
\hline Cr------------- & 60 & 57 & 57 & 60 & 55 & 53 & 62 & 56 & 57 & 47 & 46 \\
\hline Cu------------- & 19 & 15 & 16 & 19 & 16 & 14 & 18 & 16 & 18 & 15 & 16 \\
\hline Ga------------. & 7 & 7 & 7 & 7 & 6 & 6 & 7 & 7 & 7 & 7 & 6 \\
\hline La------------- & 16 & 16 & 16 & 17 & 15 & 15 & 18 & 16 & 16 & 14 & 13 \\
\hline Li------------- & 30 & 27 & 26 & 28 & 25 & 24 & 30 & 28 & 29 & 24 & 23 \\
\hline Mo------------- & 50 & 46 & 47 & 49 & 50 & 51 & 56 & 54 & 53 & 43 & 50 \\
\hline Nb------------ & 4 & 4 & 3 & 3 & 3 & 3 & 4 & 4 & 3 & 3 & 4 \\
\hline Nd------------ & 13 & 14 & 14 & 14 & 13 & 13 & 15 & 15 & 16 & 15 & 15 \\
\hline Ni------------- & 39 & 31 & 48 & 35 & 31 & 31 & 39 & 33 & 34 & 29 & 34 \\
\hline Pb------------- & 5 & 3 & 4 & 5 & 4 & 4 & 5 & 5 & 6 & 5 & 6 \\
\hline Sr------------ & 1000 & 920 & 980 & 950 & 890 & 890 & 1000 & 990 & 990 & 910 & 840 \\
\hline Th-----------. & 5 & 6 & 3 & 5 & 4 & 3 & 5 & 5 & 5 & 4 & 4 \\
\hline V-------------- & 92 & 77 & 84 & 88 & 77 & 81 & 92 & 90 & 93 & 80 & 83 \\
\hline Y----------- & 8 & 8 & 8 & 8 & 7 & 7 & 9 & 8 & 8 & 7 & 7 \\
\hline Zn-------------. & 57 & 48 & 51 & 55 & 48 & 44 & 57 & 56 & 56 & 48 & 50 \\
\hline Sample & $39 \mathrm{PC} 422$ & $39 \mathrm{PC} 427$ & $39 \mathrm{PC} 432$ & $39 \mathrm{PC} 437$ & $39 \mathrm{PC} 442$ & $39 \mathrm{PC} 447$ & $39 \mathrm{PC} 452$ & $39 \mathrm{PC} 457$ & $39 \mathrm{PC} 462$ & 39PC468 & $39 \mathrm{PC} 472$ \\
\hline Depth (cm)-- & 402 & 407 & 412 & 417 & 422 & 427 & 432 & 437 & 442 & 448 & 452 \\
\hline Age (yr)------ & 12030 & 12077 & 12123 & 12168 & 12211 & 12254 & 12296 & 12340 & 12385 & 12433 & 12483 \\
\hline $\mathrm{Al}_{2} \mathrm{O}_{3}$--------- & 5.86 & 7.56 & 6.23 & 6.61 & 6.8 & 6.8 & 7.18 & 8.12 & 8.5 & 6.8 & 7.56 \\
\hline $\mathrm{CaO}-\cdot--\cdot----$ & 15.4 & 19.6 & 18.2 & 16.8 & 18.2 & 18.2 & 22.4 & 19.6 & 19.6 & 16.8 & 16.8 \\
\hline MgO---------- & 2.16 & 2.32 & 2.16 & 2.16 & 2.16 & 2.16 & 2.32 & 2.32 & 2.49 & 2.16 & 2.32 \\
\hline $\mathrm{TiO}_{2}$ & 0.18 & 0.23 & 0.2 & 0.2 & 0.22 & 0.23 & 0.23 & 0.27 & 0.28 & 0.22 & 0.23 \\
\hline $\mathrm{K}_{2} \mathrm{O}$ & 0.94 & 1.11 & 0.96 & 1.02 & 1.02 & 1 & 1.06 & 1.16 & 1.2 & 1.04 & 1.1 \\
\hline $\mathrm{Fe}_{2} \mathrm{O}_{3}$-------- & 2 & 2.57 & 2.14 & 2.43 & 2.29 & 2.43 & 2.43 & 2.86 & 3 & 2.43 & 2.57 \\
\hline $\mathrm{P}_{2} \mathrm{O}_{5}$ & 0.16 & 0.18 & 0.18 & 0.18 & 0.18 & 0.18 & 0.21 & 0.21 & 0.21 & 0.23 & 0.21 \\
\hline MnO--------- & 101 & 155 & 118 & 120 & 119 & 121 & 142 & 168 & 181 & 128 & 142 \\
\hline As-------------- & 7 & 7 & 7 & 7 & 7 & 7 & 7 & 7 & 7 & 7 & 7 \\
\hline Ba-------------- & 150 & 190 & 160 & 160 & 190 & 160 & 180 & 200 & 200 & 150 & 180 \\
\hline Cd------------- & 3 & 3 & 3 & 3 & 5 & 4 & 4 & 4 & 4 & 2 & 3 \\
\hline Ce------------- & 21 & 29 & 25 & 27 & 26 & 28 & 29 & 32 & 35 & 27 & 29 \\
\hline Co------------- & 5 & 6 & 5 & 7 & 6 & 6 & 6 & 6 & 7 & 6 & 6 \\
\hline Cr------------- & 52 & 63 & 54 & 57 & 59 & 62 & 62 & 72 & 73 & 62 & 66 \\
\hline Cu------------- & 20 & 17 & 15 & 18 & 17 & 16 & 18 & 20 & 19 & 16 & 17 \\
\hline Ga-------------- & 7 & 8 & 7 & 8 & 9 & 8 & 9 & 10 & 10 & 8 & 9 \\
\hline La-------------- & 15 & 18 & 16 & 16 & 17 & 18 & 18 & 21 & 21 & 17 & 19 \\
\hline Li-------------. & 26 & 35 & 29 & 30 & 31 & 31 & 32 & 36 & 38 & 30 & 33 \\
\hline Mo------------- & 42 & 37 & 36 & 47 & 44 & 43 & 32 & 39 & 35 & 48 & 40 \\
\hline Nb-------------. & 3 & 4 & 3 & 3 & 3 & 4 & 4 & 5 & 4 & 4 & 4 \\
\hline Nd------------.. & 14 & 19 & 15 & 16 & 18 & 18 & 20 & 21 & 20 & 19 & 17 \\
\hline Ni------------- & 34 & 38 & 29 & 34 & 35 & 36 & 36 & 41 & 43 & 30 & 31 \\
\hline Pb-------------- & 5 & 8 & 6 & 7 & 7 & 7 & 8 & 7 & 6 & 5 & 7 \\
\hline Sr--------------. & 850 & 1000 & 970 & 870 & 970 & 1000 & 1200 & 1100 & 1100 & 910 & 960 \\
\hline Th-------------- & 6 & 6 & 4 & 5 & 6 & 5 & 5 & 6 & 6 & 4 & 5 \\
\hline V--------------- & 83 & 98 & 82 & 83 & 91 & 88 & 84 & 94 & 96 & 83 & 94 \\
\hline Y-------------- & 7 & 9 & 8 & 8 & 8 & 9 & 9 & 10 & 10 & 8 & 9 \\
\hline Zn------------- & 52 & 62 & 51 & 57 & 61 & 58 & 62 & 69 & 71 & 57 & 61 \\
\hline
\end{tabular}


Table 1. Concentrations in sediment of core PL07-39PC of major-element oxides-Continued.

\begin{tabular}{|c|c|c|c|c|c|c|c|c|c|c|c|}
\hline Sample & 39PC477 & 39PC482 & 39PC487 & 39PC492 & 39PC496 & 39PC502 & 39PC507 & 39PC512 & 39PC517 & 39PC522 & 39PC527 \\
\hline Depth (cm)-- & 457 & 462 & 467 & 472 & 476 & 482 & 487 & 492 & 497 & 502 & 507 \\
\hline Age (yr)----- & 12535 & 12590 & 12647 & 12705 & 12766 & 12828 & 12894 & 12959 & 13028 & 13098 & 13172 \\
\hline $\mathrm{Al}_{2} \mathrm{O}_{3}$ & 8.12 & 9.07 & 8.31 & 7.74 & 7.74 & 8.69 & 7.37 & 7.56 & 6.42 & 7.56 & 8.5 \\
\hline CaO---------. & 19.6 & 21 & 18.2 & 18.2 & 16.8 & 18.2 & 12.46 & 11.76 & 11.76 & 12.88 & 15.4 \\
\hline MgO--------- & 2.32 & 2.65 & 2.32 & 2.32 & 2.32 & 2.49 & 1.82 & 1.66 & 1.82 & 1.99 & 2.16 \\
\hline $\mathrm{TiO}_{2}$ & 0.27 & 0.3 & 0.27 & 0.23 & 0.25 & 0.27 & 0.22 & 0.23 & 0.2 & 0.23 & 0.25 \\
\hline $\mathrm{K}_{2} \mathrm{O}$ & 1.14 & 1.33 & 1.18 & 1.12 & 1.1 & 1.2 & 1.11 & 1.1 & 1.02 & 1.13 & 1.2 \\
\hline $\mathrm{Fe}_{2} \mathrm{O}_{3}$ & 2.86 & 3.15 & 2.86 & 2.57 & 2.72 & 3 & 2.72 & 2.86 & 2.43 & 2.72 & 3.15 \\
\hline $\mathrm{P}_{2} \mathrm{O}_{5}$ & 0.21 & 0.21 & 0.18 & 0.27 & 0.18 & 0.21 & 0.21 & 0.18 & 0.18 & 0.25 & 0.23 \\
\hline MnO--------- & 168 & 181 & 142 & 142 & 142 & 168 & 120 & 123 & 103 & 120 & 155 \\
\hline As----------- & 7 & 7 & 7 & 7 & 7 & 7 & 7 & 7 & 7 & 7 & 7 \\
\hline Ba------------ & 190 & 220 & 200 & 190 & 200 & 210 & 160 & 180 & 140 & 170 & 200 \\
\hline Cd----------.' & 4 & 3 & 2 & 1 & 1 & 2 & 3 & 8 & 7 & 6 & 4 \\
\hline Ce----------- & 33 & 35 & 31 & 29 & 31 & 32 & 27 & 28 & 27 & 29 & 30 \\
\hline 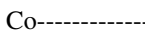 & 6 & 8 & 6 & 6 & 6 & 7 & 7 & 7 & 6 & 6 & 7 \\
\hline Cr---------- & 72 & 79 & 74 & 67 & 66 & 75 & 77 & 79 & 75 & 82 & 84 \\
\hline 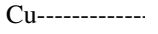 & 18 & 19 & 17 & 31 & 16 & 24 & 20 & 20 & 21 & 23 & 22 \\
\hline Ga-----------.. & 10 & 10 & 9 & 9 & 9 & 9 & 8 & 8 & 7 & 10 & 9 \\
\hline La---------- & 20 & 22 & 20 & 19 & 20 & 21 & 17 & 18 & 15 & 18 & 20 \\
\hline Li---- & 36 & 42 & 37 & 35 & 35 & 39 & 33 & 33 & 29 & 33 & 38 \\
\hline Mo------------ & 38 & 41 & 39 & 41 & 44 & 63 & 90 & 99 & 110 & 120 & 100 \\
\hline Nb---_-_---' & 4 & 5 & 5 & 4 & 4 & 4 & 4 & 3 & 3 & 4 & 5 \\
\hline Nd---------- & 22 & 22 & 20 & 19 & 19 & 20 & 16 & 15 & 14 & 16 & 22 \\
\hline Ni----------- & 35 & 41 & 34 & 32 & 32 & 36 & 43 & 47 & 45 & 48 & 45 \\
\hline Pb------------ & 7 & 9 & 6 & 8 & 8 & 8 & 8 & 8 & 7 & 10 & 7 \\
\hline Sr-----------י & 1100 & 1200 & 1000 & 1000 & 960 & 1000 & 770 & 720 & 700 & 780 & 970 \\
\hline Th------------- & 7 & 7 & 6 & 6 & 6 & 6 & 6 & 5 & 5 & 5 & 7 \\
\hline V----------- & 90 & 100 & 92 & 92 & 98 & 100 & 120 & 130 & 190 & 170 & 170 \\
\hline Y---------- & 10 & 11 & 10 & 9 & 9 & 10 & 8 & 8 & 8 & 9 & 9 \\
\hline Zn------------ & 69 & 71 & 67 & 62 & 60 & 69 & 64 & 65 & 55 & 68 & 71 \\
\hline Sample & 39PC532 & $39 \mathrm{PC} 537$ & 39PC542 & 39PC547 & 39PC552 & 39PC557 & 39PC562 & 39PC567 & 39PC572 & 39PC578 & 39PC582 \\
\hline Depth (cm)-- & 512 & 517 & 522 & 527 & 532 & 537 & 542 & 547 & 552 & 558 & 562 \\
\hline Age (yr)----- & 13247 & 13325 & 13404 & 13486 & 13570 & 13655 & 13742 & 13830 & 13920 & 14010 & 14103 \\
\hline $\mathrm{Al}_{2} \mathrm{O}_{3}$-------- & 6.8 & 6.8 & 5.48 & 6.42 & 6.42 & 3.78 & 6.42 & 6.61 & 5.86 & 7.74 & 7.37 \\
\hline CaO------ & 12.6 & 13.3 & 12.74 & 15.4 & 12.46 & 9.38 & 14 & 15.4 & 12.46 & 16.8 & 21 \\
\hline MgO--------. & 1.99 & 1.99 & 1.82 & 2.16 & 1.82 & 1.46 & 1.82 & 1.99 & 1.66 & 1.82 & 1.99 \\
\hline $\mathrm{TiO}_{2}$ & 0.22 & 0.2 & 0.17 & 0.2 & 0.2 & 0.12 & 0.18 & 0.18 & 0.17 & 0.23 & 0.22 \\
\hline $\mathrm{K}_{2} \mathrm{O}$ & 1.04 & 1.04 & 0.89 & 1.01 & 1 & 0.69 & 1 & 0.99 & 0.88 & 1.07 & 1.02 \\
\hline $\mathrm{Fe}_{2} \mathrm{O}_{3}$ & 2.43 & 2.43 & 2 & 2.43 & 2.43 & 1.43 & 2.43 & 2.57 & 2.29 & 3 & 2.72 \\
\hline $\mathrm{P}_{2} \mathrm{O}_{5}$--------- & 0.21 & 0.16 & 0.14 & 0.21 & 0.14 & 0.21 & 0.6 & 0.18 & 0.16 & 0.18 & 0.21 \\
\hline MnO--------. & 128 & 125 & 110 & 129 & 129 & 106 & 181 & 194 & 181 & 245 & 258 \\
\hline As----------. & 7 & 7 & 7 & 7 & 7 & 7 & 7 & 7 & 7 & 11 & 13 \\
\hline Ba-----------.. & 170 & 160 & 140 & 150 & 160 & 91 & 160 & 160 & 140 & 190 & 190 \\
\hline Cd----------. & 4 & 2 & 3 & 4 & 3 & 3 & 7 & 3 & 4 & 4 & 6 \\
\hline Ce----------.. & 26 & 26 & 22 & 25 & 23 & 12 & 23 & 23 & 20 & 31 & 29 \\
\hline Co---------' & 6 & 6 & 5 & 6 & 7 & 5 & 6 & 7 & 7 & 12 & 9 \\
\hline Cr----------- & 73 & 69 & 59 & 64 & 90 & 56 & 72 & 76 & 89 & 100 & 90 \\
\hline Cu--- & 19 & 17 & 16 & 17 & 22 & 15 & 21 & 20 & 25 & 25 & 27 \\
\hline Ga-----------. & 8 & 8 & 6 & 9 & 7 & 4 & 7 & 7 & 7 & 9 & 9 \\
\hline La----------- & 17 & 16 & 13 & 16 & 15 & 9 & 15 & 16 & 13 & 18 & 19 \\
\hline Li---------- & 31 & 30 & 24 & 29 & 29 & 16 & 28 & 29 & 25 & 33 & 33 \\
\hline Mo-----------. & 76 & 99 & 77 & 91 & 130 & 72 & 120 & 130 & 120 & 150 & 140 \\
\hline Nb----------- & 4 & 3 & 3 & 3 & 3 & 4 & 3 & 3 & 3 & 4 & 3 \\
\hline Nd----------- & 17 & 15 & 13 & 22 & 14 & 8 & 14 & 15 & 12 & 20 & 20 \\
\hline Ni----------- & 37 & 40 & 35 & 39 & 58 & 37 & 50 & 54 & 57 & 67 & 74 \\
\hline Pb------------ & 7 & 7 & 6 & 8 & 6 & 4 & 8 & 8 & 7 & 10 & 8 \\
\hline Sr------------- & 730 & 780 & 760 & 890 & 740 & 550 & 860 & 900 & 710 & 920 & 1100 \\
\hline Th-----------.. & 4 & 4 & 3 & 10 & 4 & 4 & 5 & 4 & 4 & 4 & 5 \\
\hline V------------ & 140 & 140 & 120 & 120 & 190 & 120 & 130 & 130 & 190 & 150 & 140 \\
\hline Y----------- & 8 & 7 & 6 & 8 & 7 & 5 & 8 & 8 & 7 & 10 & 9 \\
\hline Zn-----------.-. & 56 & 54 & 45 & 62 & 59 & 36 & 77 & 63 & 56 & 76 & 75 \\
\hline
\end{tabular}


Table 1. Concentrations in sediment of core PL07-39PC of major-element oxides-Continued.

\begin{tabular}{|c|c|c|c|c|c|c|c|c|c|c|c|}
\hline Sample & 39PC587 & 39PC592 & 39PC597 & 39PC602 & 39PC606 & 39PC612 & 39 PC617 & 39PC622 & 39 PC627 & 39PC632 & 39PC637 \\
\hline Depth (cm)-- & 567 & 572 & 577 & 582 & 586 & 592 & 597 & 602 & 607 & 612 & 617 \\
\hline Age (yr)-----. & 14196 & 14290 & 14384 & 14477 & 14571 & 14664 & 14756 & 14846 & 14936 & 15024 & 15111 \\
\hline $\mathrm{Al}_{2} \mathrm{O}_{3}$-------- & 5.86 & 8.12 & 8.88 & 7.37 & 6.8 & 8.12 & 7.18 & 3.78 & 7.93 & 11.9 & 11.71 \\
\hline $\mathrm{CaO}$ & 16.8 & 19.6 & 19.6 & 18.2 & 18.2 & 18.2 & 21 & 9.94 & 23.8 & 18.2 & 18.2 \\
\hline MgO-------.. & 1.82 & 1.82 & 1.82 & 1.82 & 1.66 & 1.66 & 1.64 & 0.88 & 2.16 & 2.49 & 2.65 \\
\hline $\mathrm{TiO}_{2}$ & 0.17 & 0.25 & 0.28 & 0.23 & 0.23 & 0.27 & 0.25 & 0.13 & 0.27 & 0.43 & 0.42 \\
\hline $\mathrm{K}_{2} \mathrm{O}$ & 0.88 & 1.06 & 1.13 & 1.04 & 0.96 & 1.1 & 0.98 & 0.52 & 1.08 & 1.57 & 1.57 \\
\hline $\mathrm{Fe}_{2} \mathrm{O}_{3}$-------- & 2 & 3 & 3.29 & 2.86 & 2.57 & 3 & 2.86 & 1.57 & 2.86 & 3.86 & 3.72 \\
\hline 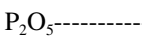 & 0.3 & 0.44 & 0.25 & 0.25 & 0.21 & 0.21 & 0.18 & 0.14 & 0.25 & 0.16 & 0.18 \\
\hline MnO------.. & 220 & 323 & 323 & 297 & 310 & 310 & 310 & 142 & 181 & 245 & 232 \\
\hline As---------..' & 7 & 7 & 11 & 7 & 10 & 7 & 7 & 7 & 7 & 15 & 12 \\
\hline Ba---------.. & 150 & 280 & 220 & 190 & 170 & 200 & 180 & 100 & 210 & 260 & 260 \\
\hline 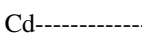 & 3 & 5 & 6 & 6 & 3 & 4 & 3 & 2 & 4 & 1 & 1 \\
\hline Ce--------.. & 21 & 32 & 36 & 28 & 27 & 30 & 26 & 16 & 34 & 45 & 42 \\
\hline Co---------.. & 7 & 12 & 12 & 10 & 9 & 11 & 12 & 9 & 10 & 12 & 11 \\
\hline Cr--------- & 76 & 86 & 94 & 92 & 86 & 98 & 83 & 45 & 84 & 100 & 98 \\
\hline Cu----------. & 23 & 27 & 30 & 28 & 27 & 28 & 26 & 13 & 28 & 35 & 33 \\
\hline Ga--------. & 8 & 9 & 10 & 9 & 8 & 10 & 8 & 4 & 10 & 14 & 13 \\
\hline La-----------. & 13 & 19 & 22 & 17 & 16 & 18 & 17 & 10 & 19 & 27 & 26 \\
\hline Li--------- & 25 & 36 & 38 & 35 & 33 & 39 & 35 & 18 & 39 & 59 & 58 \\
\hline Mo----------- & 94 & 120 & 160 & 140 & 120 & 150 & 140 & 66 & 77 & 36 & 12 \\
\hline Nb---'--- & 3 & 3 & 5 & 4 & 4 & 5 & 4 & 3 & 5 & 7 & 7 \\
\hline Nd----------- & 16 & 20 & 22 & 16 & 15 & 19 & 17 & 8 & 22 & 26 & 26 \\
\hline Ni---------- & 48 & 58 & 74 & 67 & 60 & 64 & 58 & 33 & 55 & 55 & 54 \\
\hline Pb-----------. & 8 & 8 & 9 & 7 & 9 & 9 & 7 & 5 & 7 & 11 & 11 \\
\hline Sr----------- & 900 & 1100 & 1100 & 1000 & 990 & 1000 & 1100 & 550 & 1300 & 970 & 1000 \\
\hline Th---------.. & 3 & 5 & 7 & 5 & 4 & 6 & 5 & 3 & 6 & 9 & 8 \\
\hline V----------. & 140 & 140 & 160 & 170 & 140 & 160 & 140 & 76 & 130 & 140 & 130 \\
\hline Y----------. & 7 & 11 & 11 & 9 & 8 & 9 & 10 & 7 & 13 & 15 & 15 \\
\hline Zn-------... & 61 & 83 & 90 & 77 & 77 & 87 & 80 & 44 & 85 & 110 & 110 \\
\hline Sample & 39PC642 & 39PC647 & 39PC652 & 39PC657 & 39PC662 & 39PC667 & 39PC672 & 39PC677 & 39PC682 & 39PC688 & 39PC692 \\
\hline Depth (cm)-- & 622 & 627 & 632 & 637 & 642 & 647 & 652 & 657 & 662 & 667 & 672 \\
\hline Age (yr)----- & 15197 & 15282 & 15365 & 15448 & 15530 & 15610 & 15690 & 15770 & 15849 & 15927 & 16006 \\
\hline $\mathrm{Al}_{2} \mathrm{O}_{3}$ & 10.77 & 10.77 & 11.33 & 11.33 & 11.9 & 11.52 & 10.58 & 12.47 & 14.92 & 14.17 & 14.73 \\
\hline CaO----'-- & 22.4 & 21 & 19.6 & 19.6 & 18.2 & 19.6 & 21 & 16.8 & 10.08 & 12.46 & 10.22 \\
\hline MgO--------. & 2.49 & 2.49 & 2.49 & 2.49 & 2.49 & 2.65 & 2.49 & 2.32 & 2.16 & 2.32 & 2.32 \\
\hline $\mathrm{TiO}_{2}$ & 0.38 & 0.38 & 0.43 & 0.43 & 0.45 & 0.45 & 0.38 & 0.47 & 0.62 & 0.55 & 0.62 \\
\hline $\mathrm{K}_{2} \mathrm{O}-$ & 1.45 & 1.45 & 1.57 & 1.57 & 1.57 & 1.57 & 1.45 & 1.57 & 1.81 & 1.81 & 1.81 \\
\hline $\mathrm{Fe}_{2} \mathrm{O}_{3}$ & 3.43 & 3.57 & 3.86 & 3.72 & 4 & 3.86 & 3.72 & 4.43 & 5.15 & 4.86 & 5.15 \\
\hline $\mathrm{P}_{2} \mathrm{O}_{5}$ & 0.18 & 0.16 & 0.18 & 0.16 & 0.16 & 0.16 & 0.18 & 0.14 & 0.11 & 0.14 & 0.11 \\
\hline MnO------. & 207 & 220 & 245 & 232 & 258 & 245 & 207 & 271 & 284 & 297 & 310 \\
\hline As----------. & 7 & 11 & 10 & 7 & 7 & 7 & 12 & 14 & 7 & 7 & 7 \\
\hline Ba--------. & 240 & 240 & 260 & 250 & 260 & 260 & 240 & 260 & 300 & 290 & 310 \\
\hline Cd-----------. & 1 & 1 & 1 & 1 & 1 & 1 & 1 & 1 & 1 & 1 & 1 \\
\hline Ce--י--י--. & 41 & 39 & 43 & 43 & 44 & 44 & 39 & 44 & 54 & 52 & 53 \\
\hline Co----------. & 11 & 11 & 12 & 12 & 12 & 11 & 11 & 13 & 16 & 16 & 17 \\
\hline Cr---------- & 91 & 90 & 94 & 72 & 96 & 95 & 89 & 99 & 96 & 99 & 110 \\
\hline Cu--------. & 31 & 30 & 33 & 32 & 34 & 33 & 31 & 34 & 38 & 37 & 39 \\
\hline Ga-------- & 13 & 13 & 14 & 14 & 14 & 14 & 12 & 15 & 18 & 17 & 18 \\
\hline La---------. & 25 & 24 & 26 & 26 & 26 & 26 & 24 & 27 & 32 & 31 & 32 \\
\hline Li----------- & 54 & 54 & 56 & 56 & 58 & 57 & 52 & 58 & 66 & 65 & 67 \\
\hline Mo----------. & 6 & 4 & 3 & 2 & 3 & 1 & 2 & 1 & 1 & 1 & 1 \\
\hline Nb----------- & 7 & 7 & 7 & 7 & 7 & 7 & 7 & 7 & 8 & 8 & 9 \\
\hline Nd--------- & 25 & 24 & 27 & 27 & 25 & 26 & 24 & 27 & 28 & 30 & 30 \\
\hline Ni---------- & 53 & 52 & 54 & 52 & 53 & 54 & 52 & 57 & 57 & 58 & 60 \\
\hline Pb----------. & 8 & 9 & 9 & 7 & 9 & 11 & 8 & 11 & 13 & 13 & 12 \\
\hline Sr------------- & 1100 & 1100 & 1000 & 1000 & 970 & 1000 & 1100 & 870 & 570 & 670 & 570 \\
\hline Th---------. & 8 & 8 & 8 & 7 & 7 & 8 & 7 & 8 & 10 & 10 & 9 \\
\hline V------------ & 120 & 120 & 130 & 130 & 130 & 130 & 130 & 140 & 170 & 160 & 170 \\
\hline Y---------- & 14 & 14 & 14 & 14 & 14 & 14 & 13 & 15 & 15 & 15 & 16 \\
\hline Zn-----------. & 110 & 100 & 110 & 110 & 110 & 110 & 96 & 110 & 140 & 130 & 140 \\
\hline
\end{tabular}


Table 1. Concentrations in sediment of core PL07-39PC of major-element oxides-Continued.

\begin{tabular}{|c|c|c|c|c|c|c|c|c|c|c|c|}
\hline $\begin{array}{l}\text { Sample } \\
\end{array}$ & 39PC697 & 39PC702 & 39PC706 & $39 \mathrm{PC} 712$ & 39PC717 & 39PC722 & $39 P C 727$ & $39 \mathrm{PC} 732$ & $39 \mathrm{PC} 737$ & 39PC742 & 39PC747 \\
\hline Depth (cm)-- & 677 & 682 & 686 & 692 & 697 & 702 & 707 & 712 & 717 & 722 & 727 \\
\hline Age (yr)------ & 16084 & 16162 & 16241 & 16319 & 16399 & 16479 & 16560 & 16642 & 16726 & 16810 & 16896 \\
\hline $\mathrm{Al}_{2} \mathrm{O}_{3}$ & 16.81 & 17.38 & 12.47 & 14.17 & 14.92 & 18.13 & 8.31 & 9.44 & 7.93 & 8.88 & 10.01 \\
\hline CaO--------- & 5.18 & 3.08 & 14 & 10.36 & 8.26 & 3.92 & 19.6 & 18.2 & 18.2 & 18.2 & 15.4 \\
\hline MgO--------. & 2.16 & 1.99 & 2.49 & 2.32 & 2.32 & 2.16 & 7.13 & 7.46 & 6.97 & 5.64 & 3.15 \\
\hline $\mathrm{TiO}_{2}$ & 0.75 & 0.77 & 0.5 & 0.58 & 0.62 & 0.72 & 0.32 & 0.35 & 0.32 & 0.33 & 0.4 \\
\hline $\mathrm{K}_{2} \mathrm{O}$ & 2.05 & 1.93 & 1.57 & 1.81 & 1.81 & 1.93 & 1.19 & 1.33 & 1.2 & 1.33 & 1.57 \\
\hline $\mathrm{Fe}_{2} \mathrm{O}_{3}$ & 5.72 & 5.86 & 5 & 5.29 & 5.72 & 5.86 & 3.43 & 3.43 & 3 & 3 & 3.72 \\
\hline $\mathrm{P}_{2} \mathrm{O}_{5}$ & 0.09 & 0.09 & 0.14 & 0.11 & 0.11 & 0.09 & 0.18 & 0.21 & 0.18 & 0.16 & 0.14 \\
\hline MnO-------- & 297 & 271 & 297 & 284 & 271 & 258 & 194 & 194 & 155 & 168 & 207 \\
\hline As----------. & 7 & 7 & 12 & 7 & 12 & 13 & 7 & 7 & 7 & 7 & 7 \\
\hline Ba---------. & 340 & 340 & 270 & 300 & 310 & 350 & 200 & 220 & 190 & 210 & 220 \\
\hline Cd------------. & 1 & 1 & 1 & 1 & 1 & 1 & 1 & 1 & 1 & 1 & 1 \\
\hline Ce---------- & 58 & 60 & 47 & 52 & 53 & 59 & 30 & 35 & 30 & 34 & 41 \\
\hline Co----------- & 18 & 18 & 14 & 16 & 17 & 18 & 9 & 10 & 8 & 9 & 9 \\
\hline Cr----------- & 120 & 120 & 100 & 110 & 110 & 120 & 71 & 77 & 70 & 77 & 89 \\
\hline Cu----------. & 44 & 42 & 36 & 38 & 41 & 44 & 23 & 25 & 19 & 22 & 25 \\
\hline Ga---------. & 20 & 20 & 15 & 18 & 18 & 20 & 10 & 11 & 9 & 11 & 12 \\
\hline La--------- & 34 & 35 & 28 & 30 & 31 & 35 & 18 & 20 & 18 & 20 & 24 \\
\hline Li-------- & 74 & 72 & 59 & 65 & 66 & 73 & 42 & 48 & 42 & 45 & 51 \\
\hline Mo------------ & 1 & 1 & 1 & 1 & 1 & 1 & 1 & 1 & 1 & 1 & 3 \\
\hline Nb------------ & 11 & 9 & 7 & 9 & 8 & 8 & 5 & 7 & 6 & 6 & 6 \\
\hline Nd----------- & 32 & 32 & 26 & 28 & 30 & 30 & 20 & 21 & 17 & 20 & 24 \\
\hline Ni---------- & 62 & 60 & 60 & 59 & 62 & 60 & 41 & 43 & 41 & 46 & 49 \\
\hline Pb------------ & 14 & 16 & 10 & 12 & 13 & 12 & 8 & 6 & 7 & 8 & 12 \\
\hline Sr------------- & 330 & 240 & 780 & 550 & 450 & 280 & 650 & 630 & 620 & 710 & 720 \\
\hline Th-----------. & 11 & 11 & 6 & 8 & 10 & 11 & 6 & 7 & 6 & 7 & 8 \\
\hline V------------ & 200 & 210 & 150 & 160 & 170 & 200 & 95 & 98 & 81 & 87 & 98 \\
\hline Y---------- & 17 & 15 & 16 & 16 & 16 & 15 & 10 & 10 & 10 & 11 & 13 \\
\hline Zn---------. & 150 & 150 & 120 & 130 & 130 & 150 & 67 & 77 & 65 & 72 & 87 \\
\hline Sample & 39PC752 & 39PC757 & 39PC762 & $39 P C 767$ & 39PC772 & 39PC777 & 39PC782 & $39 \mathrm{PC} 787$ & 39PC792 & $39 P C 797$ & $39 \mathrm{PC} 802$ \\
\hline Depth (cm)-- & 732 & 737 & 742 & 747 & 752 & 757 & 762 & 767 & 772 & 777 & 782 \\
\hline Age (yr)-----. & 16983 & 17072 & 17163 & 17255 & 17349 & 17445 & 17543 & 17643 & 17745 & 17848 & 17954 \\
\hline $\mathrm{Al}_{2} \mathrm{O}_{3}$ & 10.39 & 9.26 & 9.07 & 9.07 & 11.52 & 9.07 & 9.26 & 8.88 & 9.26 & 9.26 & 8.5 \\
\hline CaO------- & 12.88 & 11.48 & 11.06 & 13.44 & 15.4 & 23.8 & 19.6 & 23.8 & 23.8 & 22.4 & 25.2 \\
\hline MgO--------. & 2.49 & 1.99 & 1.99 & 1.99 & 2.49 & 2.82 & 2.65 & 3.15 & 3.32 & 3.48 & 3.65 \\
\hline $\mathrm{TiO}_{2}$ & 0.4 & 0.38 & 0.4 & 0.37 & 0.43 & 0.32 & 0.35 & 0.33 & 0.33 & 0.33 & 0.32 \\
\hline $\mathrm{K}_{2} \mathrm{O}$ & 1.57 & 1.45 & 1.45 & 1.45 & 1.57 & 1.33 & 1.45 & 1.2 & 1.2 & 1.2 & 1.14 \\
\hline $\mathrm{Fe}_{2} \mathrm{O}_{3}$ & 3.72 & 3.43 & 3.43 & 3.43 & 4 & 3.15 & 3.29 & 3 & 3.15 & 3.15 & 2.72 \\
\hline $\mathrm{P}_{2} \mathrm{O}_{5}$ & 0.14 & 0.11 & 0.11 & 0.16 & 0.14 & 0.16 & 0.16 & 0.16 & 0.16 & 0.16 & 0.16 \\
\hline MnO--------. & 207 & 168 & 168 & 181 & 271 & 207 & 194 & 207 & 232 & 232 & 207 \\
\hline As-----------. & 11 & 7 & 7 & 7 & 17 & 10 & 7 & 11 & 7 & 13 & 7 \\
\hline Ba----------.' & 230 & 200 & 200 & 200 & 250 & 200 & 200 & 190 & 200 & 210 & 180 \\
\hline Cd----------- & 1 & 1 & 1 & 1 & 1 & 1 & 1 & 1 & 1 & 1 & 1 \\
\hline 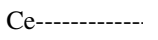 & 41 & 39 & 42 & 40 & 42 & 35 & 38 & 34 & 34 & 33 & 32 \\
\hline Co----------- & 10 & 8 & 8 & 8 & 11 & 9 & 9 & 9 & 10 & 10 & 9 \\
\hline Cr----------- & 92 & 82 & 83 & 81 & 92 & 78 & 78 & 78 & 80 & 70 & 76 \\
\hline Cu---'--- & 23 & 18 & 16 & 19 & 32 & 27 & 23 & 29 & 29 & 28 & 27 \\
\hline Ga----------- & 12 & 11 & 10 & 11 & 14 & 11 & 10 & 10 & 11 & 11 & 10 \\
\hline La-----------. & 24 & 24 & 24 & 23 & 26 & 21 & 23 & 21 & 21 & 21 & 22 \\
\hline Li---------- & 52 & 47 & 46 & 47 & 57 & 47 & 48 & 47 & 48 & 48 & 45 \\
\hline Mo----------. & 3 & 2 & 4 & 4 & 6 & 3 & 2 & 3 & 4 & 7 & 3 \\
\hline Nb---------- & 7 & 7 & 7 & 7 & 7 & 6 & 6 & 6 & 5 & 6 & 6 \\
\hline Nd---------- & 24 & 22 & 23 & 23 & 24 & 21 & 23 & 22 & 23 & 22 & 23 \\
\hline Ni------------ & 48 & 41 & 41 & 41 & 51 & 49 & 41 & 48 & 48 & 46 & 47 \\
\hline Pb-----------. & 12 & 11 & 12 & 11 & 9 & 8 & 8 & 9 & 8 & 7 & 4 \\
\hline Sr----------- & 660 & 590 & 560 & 710 & 840 & 1200 & 1000 & 1200 & 1100 & 1100 & 1200 \\
\hline Th---------.' & 7 & 8 & 9 & 7 & 7 & 6 & 7 & 6 & 6 & 7 & 7 \\
\hline V------------. & 100 & 85 & 85 & 89 & 130 & 98 & 97 & 99 & 110 & 110 & 100 \\
\hline Y-----------. & 13 & 11 & 12 & 13 & 13 & 13 & 14 & 13 & 13 & 12 & 13 \\
\hline Zn-- & 89 & 75 & 71 & 75 & 100 & 86 & 82 & 87 & 89 & 87 & 81 \\
\hline
\end{tabular}


Table 1. Concentrations in sediment of core PL07-39PC of major-element oxides-Continued.

\begin{tabular}{|c|c|c|c|c|c|c|c|c|c|c|c|}
\hline Sample & 39PC807 & 39PC812 & 39PC817 & $39 \mathrm{PC} 822$ & 39PC827 & 39PC 832 & 39PC837 & 39PC 842 & 39PC847 & 39PC852 & 39PC857 \\
\hline Depth (cm)-- & 787 & 792 & 797 & 802 & 807 & 812 & 817 & 822 & 827 & 832 & 837 \\
\hline Age (yr)-----. & 18060 & 18170 & 18281 & 18395 & 18510 & 18629 & 18748 & 18871 & 18995 & 19123 & 19253 \\
\hline $\mathrm{Al}_{2} \mathrm{O}_{3}$ & 8.69 & 8.31 & 7.93 & 8.88 & 8.12 & 7.93 & 8.31 & 8.31 & 8.31 & 8.88 & 8.5 \\
\hline CaO-------- & 25.2 & 25.2 & 26.6 & 22.4 & 25.2 & 25.2 & 25.2 & 25.2 & 23.8 & 23.8 & 25.2 \\
\hline MgO--------. & 3.81 & 4.31 & 3.98 & 3.81 & 4.15 & 4.15 & 3.98 & 3.48 & 3.48 & 3.15 & 3.15 \\
\hline $\mathrm{TiO}_{2---------}$ & 0.32 & 0.3 & 0.3 & 0.33 & 0.3 & 0.3 & 0.3 & 0.32 & 0.32 & 0.33 & 0.32 \\
\hline $\mathrm{K}_{2} \mathrm{O}$-------.. & 1.18 & 1.14 & 1.11 & 1.2 & 1.12 & 1.1 & 1.14 & 1.13 & 1.14 & 1.19 & 1.16 \\
\hline $\mathrm{Fe}_{2} \mathrm{O}_{3}$ & 2.86 & 2.86 & 2.72 & 3.15 & 2.86 & 2.72 & 2.86 & 2.86 & 3 & 3.15 & 3 \\
\hline $\mathrm{P}_{2} \mathrm{O}_{5^{-}}$ & 0.18 & 0.25 & 0.21 & 0.18 & 0.21 & 0.21 & 0.21 & 0.21 & 0.21 & 0.21 & 0.23 \\
\hline MnO--------.. & 220 & 220 & 220 & 245 & 232 & 220 & 232 & 245 & 232 & 258 & 258 \\
\hline As----------'. & 11 & 11 & 7 & 7 & 11 & 12 & 11 & 13 & 13 & 16 & 19 \\
\hline Ва----------. & 190 & 180 & 180 & 190 & 180 & 180 & 180 & 190 & 180 & 200 & 190 \\
\hline Cd------------ & 1 & 1 & 1 & 1 & 1 & 1 & 1 & 1 & 1 & 1 & 1 \\
\hline 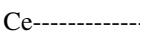 & 34 & 31 & 31 & 34 & 32 & 32 & 32 & 34 & 33 & 34 & 34 \\
\hline Co----------- & 9 & 9 & 9 & 9 & 8 & 8 & 9 & 9 & 8 & 10 & 9 \\
\hline Cr------- & 79 & 77 & 79 & 86 & 79 & 80 & 83 & 88 & 85 & 94 & 90 \\
\hline Cu---------- & 27 & 27 & 25 & 27 & 27 & 27 & 28 & 29 & 26 & 29 & 32 \\
\hline Ga--------- & 11 & 10 & 10 & 10 & 10 & 10 & 10 & 10 & 11 & 11 & 10 \\
\hline La------------. & 21 & 21 & 21 & 22 & 21 & 21 & 20 & 22 & 21 & 23 & 22 \\
\hline Li--------- & 47 & 45 & 44 & 47 & 44 & 44 & 46 & 47 & 46 & 49 & 48 \\
\hline Mo-----------. & 4 & 4 & 5 & 8 & 8 & 10 & 10 & 11 & 13 & 16 & 20 \\
\hline Nb---------- & 6 & 6 & 5 & 6 & 5 & 6 & 5 & 6 & 6 & 6 & 6 \\
\hline Nd------------ & 24 & 23 & 24 & 22 & 24 & 22 & 22 & 23 & 21 & 26 & 24 \\
\hline Ni---------- & 48 & 48 & 48 & 50 & 46 & 44 & 50 & 49 & 46 & 49 & 52 \\
\hline Pb------------. & 5 & 3 & 6 & 7 & 6 & 3 & 6 & 5 & 6 & 5 & 5 \\
\hline Sr------------- & 1200 & 1200 & 1200 & 1100 & 1200 & 1300 & 1200 & 1300 & 1200 & 1300 & 1300 \\
\hline Th-----------.. & 7 & 6 & 6 & 6 & 5 & 7 & 6 & 6 & 6 & 7 & 8 \\
\hline V-----------. & 100 & 100 & 100 & 110 & 100 & 100 & 110 & 120 & 120 & 130 & 130 \\
\hline 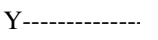 & 13 & 13 & 13 & 14 & 13 & 13 & 13 & 15 & 14 & 15 & 15 \\
\hline Zn----------. & 84 & 81 & 81 & 88 & 83 & 83 & 86 & 89 & 85 & 94 & 93 \\
\hline Sample & 39PC862 & 39PC867 & 39PC872 & 39PC877 & 39PC882 & 39PC 887 & 39PC892 & 39PC897 & 39PC902 & 39PC907 & 39PC912 \\
\hline Depth (cm)-- & 842 & 847 & 852 & 857 & 862 & 867 & 872 & 877 & 882 & 887 & 892 \\
\hline Age (yr)----. & 19386 & 19521 & 19659 & 19800 & 19945 & 20093 & 20243 & 20393 & 20543 & 20697 & 20854 \\
\hline $\mathrm{Al}_{2} \mathrm{O}_{3}$ & 8.69 & 8.88 & 9.44 & 8.88 & 8.5 & 8.69 & 8.5 & 8.5 & 8.69 & 8.5 & 8.31 \\
\hline CaO---------- & 25.2 & 23.8 & 22.4 & 23.8 & 25.2 & 23.8 & 25.2 & 25.2 & 25.2 & 25.2 & 25.2 \\
\hline MgO------. & 2.99 & 2.99 & 2.99 & 2.82 & 2.99 & 2.99 & 2.99 & 2.99 & 2.82 & 2.82 & 2.99 \\
\hline $\mathrm{TiO}_{2}$ & 0.32 & 0.32 & 0.35 & 0.32 & 0.3 & 0.32 & 0.3 & 0.32 & 0.32 & 0.32 & 0.3 \\
\hline $\mathrm{K}_{2} \mathrm{O}-$ & 1.16 & 1.18 & 1.2 & 1.17 & 1.14 & 1.17 & 1.14 & 1.14 & 1.17 & 1.13 & 1.11 \\
\hline $\mathrm{Fe}_{2} \mathrm{O}_{3}$ & 3.15 & 3.15 & 3.29 & 3 & 3 & 3.15 & 3 & 3 & 3 & 3 & 3 \\
\hline $\mathrm{P}_{2} \mathrm{O}_{5}$ & 0.23 & 0.23 & 0.21 & 0.21 & 0.23 & 0.23 & 0.21 & 0.23 & 0.21 & 0.23 & 0.21 \\
\hline MnO--------. & 258 & 258 & 258 & 245 & 245 & 245 & 232 & 232 & 232 & 245 & 232 \\
\hline As----------- & 18 & 15 & 20 & 19 & 17 & 13 & 17 & 16 & 21 & 23 & 17 \\
\hline Ba-----------.. & 190 & 190 & 210 & 190 & 190 & 190 & 190 & 190 & 190 & 180 & 180 \\
\hline Cd---------. & 1 & 1 & 2 & 1 & 1 & 1 & 1 & 1 & 1 & 1 & 1 \\
\hline Ce---------- & 34 & 34 & 37 & 35 & 32 & 34 & 32 & 33 & 34 & 33 & 33 \\
\hline Co----------. & 8 & 8 & 9 & 9 & 9 & 9 & 9 & 8 & 10 & 8 & 9 \\
\hline Cr-------- & 91 & 93 & 95 & 92 & 88 & 94 & 90 & 87 & 91 & 88 & 87 \\
\hline Cu--------. & 28 & 28 & 29 & 27 & 27 & 28 & 26 & 28 & 26 & 26 & 26 \\
\hline Ga--------. & 10 & 11 & 11 & 11 & 9 & 11 & 11 & 10 & 11 & 10 & 10 \\
\hline La--------- & 22 & 22 & 23 & 23 & 21 & 22 & 21 & 22 & 22 & 22 & 21 \\
\hline Li---'- & 49 & 49 & 51 & 48 & 48 & 49 & 48 & 48 & 49 & 48 & 48 \\
\hline Mo---------- & 25 & 24 & 27 & 25 & 28 & 22 & 23 & 30 & 24 & 19 & 21 \\
\hline Nb----.-- & 5 & 6 & 7 & 6 & 5 & 5 & 5 & 6 & 5 & 6 & 5 \\
\hline Nd---------- & 24 & 24 & 25 & 23 & 23 & 23 & 23 & 23 & 25 & 23 & 25 \\
\hline Ni---------- & 50 & 50 & 50 & 51 & 48 & 52 & 47 & 50 & 50 & 48 & 47 \\
\hline Pb--------- & 5 & 6 & 6 & 7 & 6 & 6 & 6 & 5 & 6 & 6 & 3 \\
\hline Sr---_-_--- & 1300 & 1300 & 1200 & 1200 & 1400 & 1300 & 1300 & 1300 & 1300 & 1400 & 1400 \\
\hline Th-----------. & 7 & 7 & 7 & 7 & 7 & 6 & 7 & 6 & 7 & 6 & 7 \\
\hline V---------- & 130 & 140 & 140 & 140 & 130 & 140 & 130 & 130 & 140 & 130 & 120 \\
\hline Y------------ & 14 & 15 & 15 & 14 & 14 & 14 & 14 & 13 & 14 & 14 & 14 \\
\hline Zn--------. & 92 & 94 & 96 & 94 & 90 & 93 & 89 & 90 & 93 & 90 & 87 \\
\hline
\end{tabular}


Table 1. Concentrations in sediment of core PL07-39PC of major-element oxides-Continued.

\begin{tabular}{|c|c|c|c|c|c|c|c|c|c|c|c|}
\hline Sample & 39PC917 & 39PC922 & 39PC927 & 39PC932 & 39PC937 & 39PC942 & 39PC947 & 39PC952 & 39PC957 & 39PC962 & 39PC967 \\
\hline Depth (cm)-- & 897 & 902 & 907 & 912 & 917 & 922 & 927 & 932 & 937 & 942 & 947 \\
\hline Age (yr)-----. & 21017 & 21182 & 21355 & 21528 & 21712 & 21897 & 22093 & 22292 & 22501 & 22715 & 22938 \\
\hline $\mathrm{Al}_{2} \mathrm{O}_{3}$ & 8.31 & 8.31 & 8.5 & 8.5 & 8.12 & 8.31 & 7.74 & 8.88 & 8.5 & 8.31 & 8.31 \\
\hline CaO--------- & 25.2 & 25.2 & 25.2 & 25.2 & 26.6 & 25.2 & 26.6 & 25.2 & 25.2 & 26.6 & 25.2 \\
\hline MgO--------. & 2.99 & 2.99 & 2.99 & 2.82 & 2.99 & 3.15 & 2.82 & 2.99 & 2.99 & 2.82 & 2.99 \\
\hline $\mathrm{TiO}_{2}$---------- & 0.25 & 0.3 & 0.3 & 0.3 & 0.3 & 0.3 & 0.28 & 0.32 & 0.3 & 0.3 & 0.28 \\
\hline $\mathrm{K}_{2} \mathrm{O}-\mathrm{-}-\mathrm{-}-\mathrm{-}$ & 1.11 & 1.11 & 1.12 & 1.12 & 1.08 & 1.1 & 1.04 & 1.17 & 1.11 & 1.1 & 1.08 \\
\hline $\mathrm{Fe}_{2} \mathrm{O}_{3}$------- & 2.86 & 2.86 & 2.86 & 3 & 2.86 & 2.86 & 2.86 & 3 & 3 & 2.86 & 2.86 \\
\hline $\mathrm{P}_{2} \mathrm{O}_{5^{-}}$ & 0.18 & 0.21 & 0.21 & 0.21 & 0.18 & 0.18 & 0.21 & 0.18 & 0.18 & 0.18 & 0.18 \\
\hline MnO--------. & 232 & 245 & 232 & 220 & 245 & 271 & 220 & 245 & 245 & 245 & 258 \\
\hline As------------. & 15 & 18 & 16 & 18 & 14 & 14 & 20 & 15 & 20 & 17 & 15 \\
\hline Ba-----------. & 180 & 180 & 180 & 190 & 180 & 180 & 170 & 190 & 180 & 180 & 180 \\
\hline Cd------------ & 1 & 1 & 1 & 2 & 1 & 1 & 1 & 1 & 1 & 1 & 1 \\
\hline Ce------------. & 28 & 32 & 33 & 34 & 33 & 32 & 30 & 36 & 34 & 34 & 32 \\
\hline Co------------ & 9 & 8 & 8 & 9 & 8 & 8 & 8 & 9 & 8 & 8 & 8 \\
\hline Cr------------ & 86 & 87 & 89 & 87 & 83 & 88 & 80 & 91 & 88 & 87 & 87 \\
\hline Cu------------. & 25 & 26 & 26 & 26 & 26 & 25 & 24 & 27 & 26 & 26 & 26 \\
\hline Ga------------. & 10 & 10 & 10 & 10 & 10 & 10 & 9 & 10 & 10 & 10 & 10 \\
\hline La-------------. & 19 & 21 & 22 & 22 & 20 & 22 & 20 & 23 & 22 & 21 & 21 \\
\hline Li------------- & 48 & 47 & 48 & 48 & 46 & 48 & 45 & 51 & 48 & 47 & 47 \\
\hline Mo------------. & 17 & 17 & 17 & 27 & 18 & 14 & 19 & 19 & 21 & 19 & 19 \\
\hline Nb------------ & 4 & 5 & 6 & 5 & 6 & 6 & 5 & 5 & 5 & 6 & 5 \\
\hline Nd------------ & 20 & 24 & 24 & 24 & 24 & 23 & 23 & 24 & 24 & 24 & 23 \\
\hline Ni------------- & 45 & 46 & 44 & 47 & 44 & 43 & 47 & 50 & 47 & 46 & 49 \\
\hline Pb------------. & 5 & 4 & 5 & 6 & 4 & 4 & 5 & 6 & 4 & 6 & 6 \\
\hline Sr------------- & 1400 & 1400 & 1400 & 1300 & 1400 & 1400 & 1500 & 1400 & 1400 & 1400 & 1400 \\
\hline Th------------. & 5 & 6 & 6 & 6 & 6 & 6 & 6 & 7 & 6 & 6 & 6 \\
\hline V--------------. & 120 & 120 & 120 & 130 & 120 & 110 & 110 & 130 & 120 & 120 & 110 \\
\hline Y-----------. & 13 & 14 & 14 & 14 & 14 & 15 & 13 & 15 & 14 & 14 & 14 \\
\hline Zn------------. & 85 & 86 & 89 & 89 & 85 & 86 & 80 & 92 & 88 & 86 & 88 \\
\hline
\end{tabular}

\begin{tabular}{|c|c|c|c|c|}
\hline Sample & 39PC972 & 39PC977 & 39PC982 & 39PC986 \\
\hline Depth (cm)-- & 952 & 957 & 962 & 966 \\
\hline Age (yr)----. & 23167 & 23404 & 23642 & 23879 \\
\hline $\mathrm{Al}_{2} \mathrm{O}_{3}$ & 7.74 & 6.99 & 8.5 & 8.31 \\
\hline CaO--------- & 26.6 & 30.8 & 25.2 & 25.2 \\
\hline MgO---------. & 2.82 & 2.49 & 2.99 & 3.15 \\
\hline $\mathrm{TiO}_{2}$ & 0.28 & 0.25 & 0.3 & 0.3 \\
\hline $\mathrm{K}_{2} \mathrm{O}$---------. & 1.04 & 0.93 & 1.11 & 1.11 \\
\hline $\mathrm{Fe}_{2} \mathrm{O}_{3}$ & 2.86 & 2.43 & 3 & 2.86 \\
\hline $\mathrm{P}_{2} \mathrm{O}_{5}$ & 0.21 & 0.16 & 0.18 & 0.18 \\
\hline $\mathrm{MnO}$ & 245 & 194 & 271 & 245 \\
\hline As------------. & 14 & 10 & 19 & 17 \\
\hline Ba------------. & 170 & 160 & 180 & 180 \\
\hline Cd------------ & 1 & 1 & 1 & 1 \\
\hline Ce------------. & 31 & 27 & 32 & 33 \\
\hline Co----------- & 9 & 7 & 9 & 8 \\
\hline Cr------------- & 82 & 73 & 87 & 87 \\
\hline Cu------------ & 24 & 23 & 26 & 26 \\
\hline Ga------------ & 10 & 9 & 11 & 9 \\
\hline La------------. & 20 & 18 & 22 & 22 \\
\hline Li------------- & 45 & 40 & 48 & 48 \\
\hline Mo-----------. & 18 & 14 & 21 & 19 \\
\hline Nb------------- & 5 & 5 & 5 & 6 \\
\hline Nd------------- & 23 & 21 & 24 & 24 \\
\hline Ni------------- & 45 & 36 & 47 & 49 \\
\hline Pb------------. & 4 & 4 & 6 & 4 \\
\hline Sr------------- & 1500 & 1600 & 1400 & 1400 \\
\hline Th------------. & 6 & 5 & 6 & 7 \\
\hline V--------------. & 110 & 95 & 120 & 110 \\
\hline Y-------------. & 13 & 12 & 14 & 14 \\
\hline Zn------------. & 81 & 78 & 87 & 84 \\
\hline
\end{tabular}

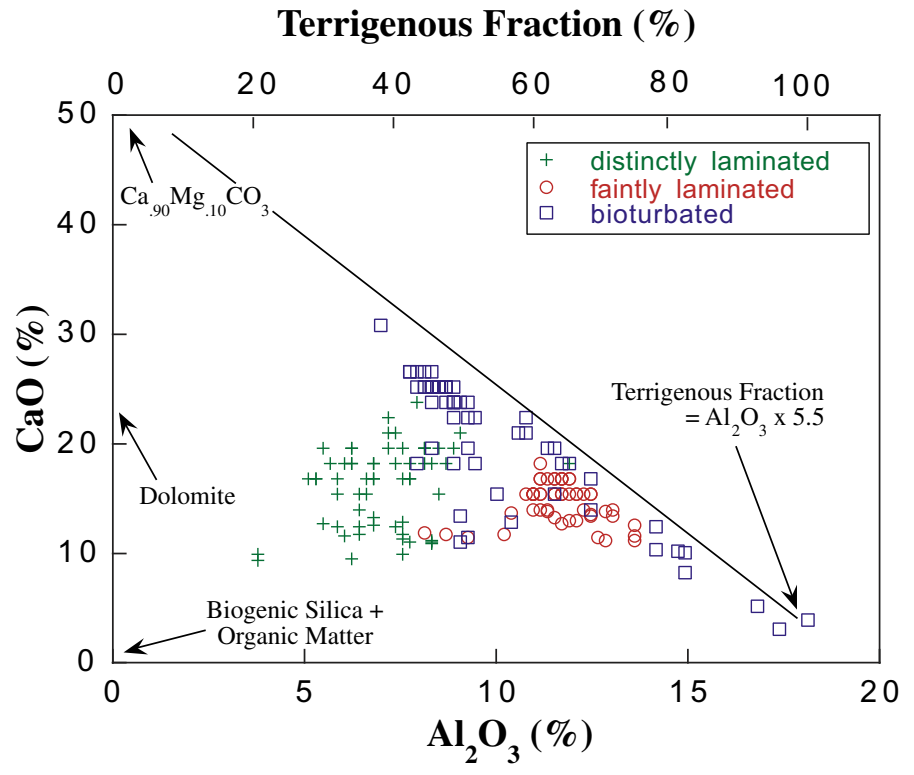

Figure 4. Relation between the concentrations of $\mathrm{Al}_{2} \mathrm{O}_{3}$ and $\mathrm{CaO}$ in core PL07-39PC. The curve represents a mixing curve for $\mathrm{CaCO}_{3}$ ( $\mathrm{CaO}$ content of 50.7 percent) and the terrigenous fraction $\left(\mathrm{Al}_{2} \mathrm{O}_{3}\right.$ content of 18.1 percent), shifted up scale to intersect the $y$-axis at the $\mathrm{CaO}$ concentration of calcite. The amount that a sample is displaced below the curve reflects its content of biogenic $\mathrm{SiO}_{2}$, organic matter, and dolomite. 
rigenous in origin (Yarincik and others, 2000). A curve drawn through these values is shifted up scale to intersect the $\mathrm{CaO}$ axis at 56 percent, the concentration of $\mathrm{CaO}$ in pure calcite. $\mathrm{X}$-ray diffraction analyses of four of the samples (James Hein, personal commun.) have shown that the calcite contains a small amount of $\mathrm{Mg}$, requiring a slight downward adjustment of the curve. The curve extrapolates to an $\mathrm{Al}_{2} \mathrm{O}_{3}$ content in the aluminosilicate fraction of 18.1 percent at a $\mathrm{CaO}$ content in the terrigenous fraction of 3.6 percent, the $\mathrm{CaO}$ concentration for the world shale average, or WSA (table 2; Wedepohl, 19691978). The $\mathrm{Al}_{2} \mathrm{O}_{3}$ concentration is greater than the mean of 15.5 percent for the WSA, but it is within the range of values used to establish the mean (Wedepohl, 1969-1978; Clarke, 1924). Thus, the conversion factor for the terrigenous fraction is 5.5 , or $(100 / 18.1) \times \mathrm{Al}_{2} \mathrm{O}_{3}$.

Offset of samples from the adjusted curve of $\mathrm{Al}_{2} \mathrm{O}_{3}$ and $\mathrm{CaO}$ largely reflects their content of organic matter and biogenic $\mathrm{SiO}_{2}$ (opal-A). Biogenic $\mathrm{SiO}_{2}$ accounts for most of the offset of samples from the distinctly laminated unit (table 3; fig. 4), having a relatively high concentration only within this unit of the core (Peterson and others, 1995). Dolomite has a very limited distribution (table 3 ). The concentration of organic carbon in the laminated sections is approximately 5 percent (Parker and Sackett, 1976; D. W. Murray, unpub. data; table 3), but exhibiting a minimum between 500 and 350 cmbsf. It is approximately 3 percent in the bioturbated section, except for several samples immediately below the distinctly laminated section, which have less than 1 percent organic carbon. Of course, organic matter, rather than merely organic carbon, determines the offset due to this component of sediment. The relation between organic carbon and organic matter in particulate matter within the water column can approximate the stoichiometry of plankton (Copin-Montegut and CopinMontegut, 1983), but the relation between the two within the sediment is likely strongly variable. For the Cariaco Basin, it has not been ascertained. A factor of 2 is arbitrarily used here to convert organic carbon to organic matter. It can be as little as 1.5 in ancient marine deposits (Powell and others, 1975).

The composition of the terrigenous fraction, as opposed to its concentration, is evaluated by examining the concentrations of $\mathrm{TiO}_{2}, \mathrm{~K}_{2} \mathrm{O}$, and $\mathrm{Fe}_{2} \mathrm{O}_{3}$ (fig. 5). Their concentrations in this fraction are determined from plots of their concentrations versus the concentration of $\mathrm{Al}_{2} \mathrm{O}_{3} \times 5.5$, or the bulk terrigenous fraction (fig. 4). All three exhibit positive correlations with the terrigenous fraction (table 4). The similarity of their concentrations with their concentrations in WSA (table 2), obtained by extrapolation of the regression curve to 100 percent terrigenous fraction and to the origin (fig. 5), supports a solely terrigenous source for these major oxides.

The concentrations of $\mathrm{TiO}_{2}$ and $\mathrm{K}_{2} \mathrm{O}$, nonetheless, show subtle differences within the terrigenous fraction of the three separate lithologic sections. Their ratios to $\mathrm{Al}_{2} \mathrm{O}_{3}$ in core PL07-39PC show extremes in the bioturbated section and the faintly laminated section. Yarincik and others (2000) attribute the differences to different sources for the terrigenous debris-(1) dominantly local rivers for the bioturbated section and (2) a mix of debris from local rivers, the Orinoco River and Amazon River systems, and eolian material from the Sahara Desert for the faintly laminated section. The Orinoco River and Amazon River systems now swamp the contribution of the other sources (Clayton and others, 1999) and have done so throughout deposition of much of the faintly laminated section of the core. An unstated assumption made by Yarincik and others (2000), and one made here also, is that this fraction of sediment is nonreactive. It has maintained its composition during its rain onto the sea floor (deposition) and burial (accumulation). Clayton and others (1999) report a similar trend in the clay mineralogy of the sediment. Such differences in both major-element-oxide concentrations and clay-mineral abundances, between lithologic units, contribute to the scatter in the data. The distribution of samples along the regression curves (fig. 5), however, reflects simple mixing of the terrigenous debris with the major biogenic phases-biogenic $\mathrm{SiO}_{2}$, calcite, and organic matter.

The plot of $\mathrm{Fe}_{2} \mathrm{O}_{3}$ concentrations along a single curve (fig. $5 C$ ) indicates a constant $\mathrm{Fe}_{2} \mathrm{O}_{3}$ concentration in the terrigenous fraction. A seawater contribution of $\mathrm{Fe}$, precipitating from pore water possibly as pyrite (Lyons, 1996) or by settling organic matter, is masked by Fe contributed by the siliciclastic debris. The coarseness of the normative calculation simply does not allow identification of a marine Fe fraction. Also, it does not preclude that some portion of the Fe, originally contributed by terrigenous debris, might now be present as pyrite.

The relation between a first group of trace elements and the terrigenous fraction is similar to the relations between $\mathrm{TiO}_{2}, \mathrm{~K}_{2} \mathrm{O}$, and $\mathrm{Fe}_{2} \mathrm{O}_{3}$ and the terrigenous fraction. The group includes $\mathrm{Ba}, \mathrm{Ce}, \mathrm{Co}, \mathrm{Ga}, \mathrm{La}, \mathrm{Li}$, and Th (fig. 6). Their concentrations are established by mixing of the terrigenous fraction with marine fractions. That is, the curves extrapolate to the origin and to WSA values at 100 percent terrigenous debris. $\mathrm{Li}$ and $\mathrm{La}$ resemble $\mathrm{K}_{2} \mathrm{O} ; \mathrm{Ga}$ and Th resemble $\mathrm{Fe}_{2} \mathrm{O}_{3}$; and Co resembles $\mathrm{TiO}_{2}$. $\mathrm{Ba}$ is enriched above a solely terrigenous contribution in several samples from the faintly laminated unit, between 175 and $200 \mathrm{cmbsf}$. Otherwise, its distribution resembles that of $\mathrm{K}_{2} \mathrm{O}$. R-mode factor analysis further shows these trace elements have high loadings on the same factor as terrigenous debris (table 5), defined by the $\mathrm{Al}_{2} \mathrm{O}_{3}$ concentrations.

Manganese exhibits a slight enrichment in several samples from two core sections (fig. 7), between 530 and $690 \mathrm{cmbsf}$ and greater than $790 \mathrm{cmbsf}$. Several of these samples also have Co contents above the value that can be assigned solely to the terrigenous fraction (fig. 6). These enrichments might be attributable to a hydrogenous fraction that accumulated under oxic bottom-water conditions and was redistributed after deposition of the overlying sediment under $\mathrm{SO}_{4}{ }^{2-}$-reducing conditions (see below). However, the variation in composition of the terrigenous fraction, noted above, and the weakness of these enrichments above the terrigenous contribution impose a major uncertainty on interpretations of the enrichments above a terrigenous contribution.

Mo and Cd constitute a second group of trace elements (fig. 8). They are strongly enriched above a terrigenous contribution throughout the laminated sections of the core. A relatively large error in assessing the terrigenous contribution 
Table 2. Concentrations of major-element oxides and trace elements (1) in the world shale average (WSA) and the terrigenous fraction of the Cariaco Basin, (2) in marine biogenic debris, and (3) in seawater.

[Values in parentheses give concentrations in the terrigenous fraction of Cariaco Basin sediment (column 2), concentrations in suspended organic matter calculated from trace-element depth profiles in the Caribbean Sea (column5), and concentrations in seawater of the Caribbean Basin at 1,000-m depth. Seawater concentrations not in parentheses are of open-ocean seawater from 2000-m depth in the Pacific 0cean. Dashes indicate that data are not available or are not considered in this study]

\begin{tabular}{|c|c|c|c|c|c|}
\hline & \multirow{2}{*}{$\begin{array}{c}\text { Terrigenous Fraction } \\
\text { WSA }^{1} / \text { Cariaco } \\
\text { Basin }^{2}\end{array}$} & \multicolumn{3}{|c|}{ Marine Fractions } & \multirow{2}{*}{$\begin{array}{c}\text { Seawater }^{6} \\
\text { Pacific / } \\
\text { Caribbean }\end{array}$} \\
\hline & & Biogenic $\mathrm{SiO}_{2}{ }^{3}$ & Biogenic Calcite ${ }^{4}$ & $\begin{array}{c}\text { Seawater Sus- } \\
\text { pended Organic } \\
\text { Matter }^{5}\end{array}$ & \\
\hline \multicolumn{6}{|c|}{ Major-Element Oxides } \\
\hline $\mathrm{P}_{2} \mathrm{O}_{5}$ & 0.16 & - & - & 1.74 & - \\
\hline $\mathrm{TiO}_{2}$ & $0.77(0.61)$ & - & - & - & - \\
\hline $\mathrm{K}_{2} \mathrm{O}-$ & $3.2(2.6)$ & - & - & - & - \\
\hline CaO-- & $3.6(3.6)$ & - & 50.7 & - & - \\
\hline $\mathrm{Fe}_{2} \mathrm{O}_{3}$ & $6.75(6.65)$ & - & - & 0.05 & - \\
\hline $\mathrm{Al}_{2} \mathrm{O}_{3}$ & $15.5(18.1)$ & - & - & - & - \\
\hline $\mathrm{SiO}_{2}$ & $58.4(58.4)$ & 100.0 & - & - & - \\
\hline Organic C------- & - & - & - & 36 & - \\
\hline \multicolumn{6}{|c|}{ Trace Elements } \\
\hline La--_-_-_-_-- & $41(41)$ & 5.8 & 0.13 & 0.14 & - \\
\hline Ce-- & $83(64)$ & 8.2 & 0.11 & 0.23 & - \\
\hline Nd---_- & $38(32)$ & 6.7 & 0.11 & 0.30 & - \\
\hline Ba-- & $410(390)$ & - & - & - & -- \\
\hline Cu---_-_-_------- & $34(33)$ & - & 0.70 & $11(7.5)$ & $0.18(0.16)$ \\
\hline 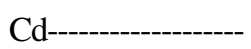 & $0.2(0.2)$ & - & - & $12(5.5)$ & $0.1(0.026)$ \\
\hline 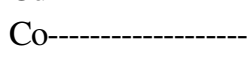 & $19.0(19)$ & - & - & 1.0 & 0.0012 \\
\hline Cr-- & $83(120)$ & - & - & 2.0 & 0.22 \\
\hline 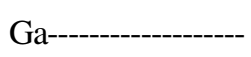 & $22(21.5)$ & - & - & - & - \\
\hline Li-- & $80(82)$ & - & - & - & - \\
\hline 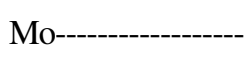 & $2.0(2.0)$ & - & - & 2.0 & 10.7 \\
\hline Ni-- & $44(65)$ & - & - & $7.5(18)$ & $0.59(0.26)$ \\
\hline 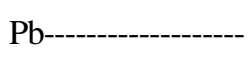 & $15(12)$ & - & - & - & - \\
\hline 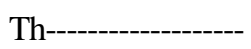 & $12(12)$ & - & - & - & -- \\
\hline V--1-1- & $120(170)$ & - & - & 3.0 & 1.8 \\
\hline Zn-1- & $100(100)$ & - & - & $110(30)^{7}$ & $0.52[0.098]$ \\
\hline
\end{tabular}

Sources of information are the following:

${ }^{1}$ Wedepohl (1969-78), Piper and Isaacs (1996).

${ }^{2}$ Figures 3 through 8 , this study.

${ }^{3}$ Elderfield and others (1981).

${ }^{4} \mathrm{Palmer}$ (1985); its $\mathrm{CaO}$ concentration represents its value in sediment examined in this study.

${ }^{5}$ Redfield and others (1963) for $\mathrm{C}$ and $\mathrm{P}_{2} \mathrm{O}_{5}$ Brumsack (1986), Piper (1994).

${ }^{6}$ Bruland (1983), for trace elements in the Pacific Ocean at 1,000- to 2,000-m depth; values in parentheses are for the Caribbean Sea, from Jacobs and others (1987); value in brackets is for the North Atlantic Ocean, from Bruland and Franks (1983).

${ }^{7} \mathrm{The} \mathrm{Zn}$ value of $30 \mathrm{ppm}$ was calculated from the $\mathrm{Zn}$ depth profile in the North Atlantic Ocean, relative to the phosphate profile (Bruland and Franks, 1983). 
Table 3. Major components in sediment samples from the Cariaco Basin, in percent, calculated from major-oxide concentrations (table 1). [Normative calculations are discussed in detail in Piper and Isaacs $(1994,1996)$. Biogenic $\mathrm{SiO}_{2}$, not listed, can only be estimated from 100 percent minus the sum. Blanks indicate no data are available]

\begin{tabular}{|c|c|c|c|c|c|c|c|c|}
\hline Sample & $\begin{array}{c}\text { Depth } \\
(\mathrm{cm})\end{array}$ & $\begin{array}{c}\text { Siliciclastic } \\
\text { Debris }^{1} \\
\end{array}$ & Calcite $^{1}$ & Dolomite $^{1}$ & Apatite $^{1}$ & $\begin{array}{c}\text { Organic } \\
\text { Matter }^{1}\end{array}$ & Sum & $\begin{array}{l}\text { Density } \\
\left(\mathrm{g} \mathrm{cm}^{-3}\right) \\
\end{array}$ \\
\hline 39PC004----- & 4 & 60.3 & 24.3 & 0.3 & 0.6 & 10.4 & 96 & 0.273 \\
\hline 39PC008----- & 9 & 60.3 & 24.3 & 0.2 & 0.3 & 11.4 & 96 & 0.282 \\
\hline 39PC013----- & 13 & 59.3 & 27.1 & 0.0 & 0.4 & & & 0.304 \\
\hline 39PC018----- & 18 & 61.4 & 24.2 & 0.1 & 0.3 & 10.5 & 97 & 0.319 \\
\hline 39PC024----- & 24 & 62.4 & 23.9 & 0.1 & 0.3 & & & 0.314 \\
\hline 39PC025----- & 26 & 62.4 & 24.2 & 0.0 & 0.3 & & & 0.318 \\
\hline 39PC034----- & 34 & 60.3 & 27.1 & 0.0 & 0.3 & 11.8 & 100 & 0.364 \\
\hline 39PC038----- & 38 & 60.3 & 27.1 & 0.0 & 0.3 & & & 0.375 \\
\hline 39PC042----- & 42 & 60.3 & 27.1 & 0.0 & 0.3 & 11.3 & 99 & 0.362 \\
\hline 39PC047----- & 47 & 63.4 & 26.9 & 0.0 & 0.3 & & & 0.388 \\
\hline 39PC052---- & 52 & 60.3 & 27.1 & 0.0 & 0.5 & 10.9 & 99 & 0.374 \\
\hline 39PC057----- & 57 & 61.4 & 27.0 & 0.0 & 0.5 & & & 0.365 \\
\hline 39PC062----- & 62 & 60.3 & 27.1 & 0.0 & 0.3 & 10.3 & 98 & 0.372 \\
\hline 39PC067----- & 67 & 61.4 & 29.8 & 0.0 & 0.4 & & & 0.39 \\
\hline 39PC072----- & 72 & 61.4 & 27.0 & 0.0 & 0.7 & 10.6 & 100 & 0.376 \\
\hline 39PC077----- & 77 & 61.4 & 27.0 & 0.0 & 0.3 & & & 0.401 \\
\hline 39PC082---- & 82 & 60.3 & 27.1 & 0.0 & 0.4 & 12.0 & 100 & 0.401 \\
\hline 39PC087----- & 87 & 62.4 & 29.8 & 0.0 & 0.4 & & & 0.413 \\
\hline 39PC092----- & 92 & 63.4 & 26.9 & 0.0 & 0.7 & 9.6 & 101 & 0.421 \\
\hline 39PC097----- & 97 & 61.4 & 29.8 & 0.0 & 0.3 & & & 0.428 \\
\hline 39PC102----- & 102 & 63.4 & 29.7 & 0.0 & 0.4 & 9.0 & 103 & 0.447 \\
\hline 39PC107----- & 107 & 64.5 & 26.8 & 0.0 & 0.3 & & & 0.474 \\
\hline 39PC112----- & 112 & 65.5 & 26.8 & 0.0 & 0.3 & 8.3 & 101 & 0.471 \\
\hline 39PC117----- & 117 & 65.5 & 29.6 & 0.0 & 0.3 & & & 0.475 \\
\hline 39PC122----- & 122 & 64.5 & 29.6 & 0.0 & 0.4 & 7.8 & 102 & 0.464 \\
\hline 39PC127----- & 127 & 64.5 & 26.8 & 0.0 & 0.6 & & & 0.467 \\
\hline 39PC132----- & 132 & 66.6 & 26.7 & 0.0 & 0.3 & 9.2 & 103 & 0.489 \\
\hline 39PC137----- & 137 & 61.4 & 32.6 & 0.0 & 0.3 & & & 0.489 \\
\hline 39PC142----- & 142 & 64.5 & 29.6 & 0.0 & 0.4 & 8.9 & 103 & 0.511 \\
\hline 39PC147----- & 147 & 66.6 & 26.7 & 0.0 & 0.3 & & & 0.508 \\
\hline 39PC152----- & 152 & 64.5 & 29.6 & 0.0 & 0.5 & 8.1 & 103 & 0.506 \\
\hline 39PC157----- & 157 & 65.5 & 29.6 & 0.0 & 0.3 & & & 0.513 \\
\hline 39PC162----- & 162 & 63.4 & 29.7 & 0.0 & 0.5 & 8.5 & 102 & 0.518 \\
\hline 39PC195----- & 175 & 68.6 & 26.6 & 0.0 & 0.4 & & & \\
\hline 39PC202----- & 182 & 67.6 & 26.6 & 0.0 & 0.3 & & & 0.633 \\
\hline 39PC207----- & 187 & 68.6 & 22.5 & 0.0 & 0.3 & & & 0.552 \\
\hline 39PC212----- & 192 & 71.8 & 26.6 & 0.0 & 0.3 & & & 0.574 \\
\hline 39PC217----- & 197 & 68.6 & 23.6 & 0.0 & 0.2 & 7.2 & 100 & 0.589 \\
\hline 39PC222----- & 202 & 71.8 & 23.4 & 0.0 & 0.3 & & & 0.568 \\
\hline 39PC227----- & 207 & 70.7 & 22.7 & 0.0 & 0.3 & 7.1 & 101 & 0.583 \\
\hline 39PC232----- & 212 & 67.6 & 20.9 & 0.0 & 0.4 & & & 0.576 \\
\hline
\end{tabular}


Table 3. Major components in sediment samples from the Cariaco Basin, in percent, calculated from major-oxide concentrations (table 1)-Continued.

\begin{tabular}{|c|c|c|c|c|c|c|c|c|}
\hline Sample & $\begin{array}{l}\text { Depth } \\
\text { (cm) }\end{array}$ & $\begin{array}{c}\text { Siliciclastic } \\
\text { Debris }\end{array}$ & Calcite & Dolomite & Apatite & $\begin{array}{l}\text { Organic } \\
\text { Matter }\end{array}$ & Sum & $\begin{array}{l}\text { Density } \\
\left(\mathrm{g} \mathrm{cm}^{-3}\right)\end{array}$ \\
\hline 39PC237----- & 217 & 74.9 & 18.6 & 0.0 & 0.2 & 8.7 & 102 & 0.562 \\
\hline 39PC242----- & 222 & 68.6 & 17.8 & 0.0 & 0.5 & & & 0.589 \\
\hline 39PC247----- & 227 & 74.9 & 18.0 & 0.0 & 0.2 & 9.5 & 103 & 0.551 \\
\hline 39PC252----- & 232 & 74.9 & 18.7 & 0.0 & 0.1 & & & 0.563 \\
\hline 39PC257----- & 237 & 70.7 & 22.0 & 0.0 & 0.3 & 9.4 & 102 & 0.574 \\
\hline 39PC262----- & 242 & 69.7 & 21.9 & 0.0 & 0.2 & & & 0.555 \\
\hline 39PC267----- & 247 & 65.5 & 26.9 & 0.0 & 0.2 & 7.6 & 100 & 0.544 \\
\hline 39PC272----- & 252 & 66.6 & 21.5 & 0.0 & 0.3 & & & 0.518 \\
\hline 39PC277----- & 257 & 63.4 & 22.7 & 0.0 & 0.3 & 8.9 & 95 & 0.541 \\
\hline 39PC282----- & 262 & 64.5 & 23.9 & 0.0 & 0.3 & & & 0.551 \\
\hline 39PC287----- & 267 & 63.4 & 20.1 & 0.0 & 0.3 & 9.5 & 93 & 0.521 \\
\hline 39PC292----- & 272 & 57.2 & 20.6 & 0.0 & 0.5 & & & 0.489 \\
\hline 39PC297----- & 277 & 56.2 & 21.0 & 0.0 & 0.3 & 9.5 & 87 & 0.477 \\
\hline 39PC302----- & 282 & 47.8 & 19.8 & 0.7 & 1.2 & & & 0.406 \\
\hline 39PC307----- & 287 & 44.7 & 19.6 & 0.9 & 1.3 & 9.3 & 76 & 0.405 \\
\hline 39PC311----- & 291 & 51.0 & 19.0 & 0.4 & 0.3 & & & 0.403 \\
\hline 39PC317----- & 297 & 45.8 & 19.3 & 0.9 & 0.3 & 9.5 & 76 & 0.456 \\
\hline 39PC322----- & 302 & 45.8 & 17.3 & 1.0 & 0.3 & & & 0.391 \\
\hline 39PC327----- & 307 & 45.8 & 19.5 & 1.0 & 0.4 & 8.3 & 75 & 0.351 \\
\hline 39PC332----- & 312 & 41.6 & 16.9 & 0.8 & 0.2 & & & 0.371 \\
\hline 39PC338----- & 318 & 42.6 & 20.1 & 0.3 & 0.2 & 9.4 & 73 & 0.399 \\
\hline 39PC343----- & 323 & 34.3 & 21.2 & 1.7 & 0.3 & & & 0.382 \\
\hline 39PC347----- & 327 & 41.6 & 31.3 & 1.2 & 0.5 & 7.8 & 82 & 0.311 \\
\hline 39PC353----- & 333 & 33.3 & 36.8 & 1.7 & 0.4 & & & 0.311 \\
\hline 39PC357----- & 337 & 37.4 & 37.1 & 1.7 & 0.4 & 6.5 & 83 & 0.346 \\
\hline 39PC362----- & 342 & 39.5 & 34.5 & 0.3 & 0.5 & & & 0.388 \\
\hline 39PC367----- & 347 & 34.3 & 37.3 & 0.8 & 0.5 & 6.0 & 79 & 0.453 \\
\hline 39PC372----- & 352 & 31.2 & 34.3 & 0.7 & 0.3 & & & 0.449 \\
\hline 39PC377----- & 357 & 30.2 & 31.8 & 0.2 & 0.4 & 5.3 & 68 & 0.411 \\
\hline 39PC382----- & 362 & 33.3 & 31.9 & 0.6 & 0.6 & & & 0.458 \\
\hline 39PC387----- & 367 & 29.1 & 37.1 & 1.5 & 0.4 & 4.9 & 73 & 0.428 \\
\hline 39PC392----- & 372 & 28.1 & 37.1 & 1.6 & 0.3 & & & 0.404 \\
\hline 39PC397----- & 377 & 34.3 & 34.3 & 0.0 & 0.3 & 5.3 & 74 & 0.428 \\
\hline 39PC402----- & 382 & 34.3 & 31.8 & 0.7 & 0.3 & & & 0.487 \\
\hline 39PC408----- & 387 & 34.3 & 31.8 & 0.5 & 0.3 & 4.9 & 72 & 0.518 \\
\hline 39PC412----- & 392 & 29.1 & 28.8 & 1.5 & 0.4 & & & 0.501 \\
\hline 39PC417----- & 397 & 29.1 & 36.6 & 1.5 & 0.4 & 4.9 & 73 & 0.403 \\
\hline 39PC422----- & 402 & 32.2 & 34.3 & 1.9 & 0.3 & & & 0.343 \\
\hline 39PC427----- & 407 & 41.6 & 31.4 & 0.1 & 0.3 & 4.1 & 77 & 0.434 \\
\hline 39PC432----- & 412 & 34.3 & 34.1 & 0.5 & 0.3 & & & 0.548 \\
\hline 39PC437----- & 417 & 36.4 & 34.1 & 0.9 & 0.3 & 5.1 & 77 & 0.451 \\
\hline 39PC442----- & 422 & 37.4 & 42.4 & 0.2 & 0.3 & & & 0.448 \\
\hline 39PC447----- & 427 & 37.4 & 36.4 & 0.2 & 0.3 & 4.3 & 79 & 0.512 \\
\hline
\end{tabular}


Table 3. Major components in sediment samples from the Cariaco Basin, in percent, calculated from major-oxide concentrations (table 1)-Continued.

\begin{tabular}{|c|c|c|c|c|c|c|c|c|}
\hline Sample & $\begin{array}{l}\text { Depth } \\
(\mathrm{cm})\end{array}$ & $\begin{array}{c}\text { Siliciclastic } \\
\text { Debris }\end{array}$ & Calcite & Dolomite & Apatite & $\begin{array}{l}\text { Organic } \\
\text { Matter }\end{array}$ & Sum & $\begin{array}{l}\text { Density } \\
\left(\mathrm{g} \mathrm{cm}^{-3}\right) \\
\end{array}$ \\
\hline 39PC452----- & 432 & 39.5 & 36.3 & 0.0 & 0.4 & & & $\overline{0.466}$ \\
\hline 39PC457----- & 437 & 44.7 & 31.3 & 0.0 & 0.3 & 5.3 & 82 & 0.472 \\
\hline 39PC462----- & 442 & 46.8 & 31.0 & 0.6 & 0.3 & & & 0.576 \\
\hline 39PC468----- & 448 & 37.4 & 36.4 & 0.9 & 0.4 & 4.5 & 80 & 0.572 \\
\hline 39PC472----- & 452 & 41.6 & 38.9 & 1.4 & 0.4 & & & 0.457 \\
\hline 39PC477----- & 457 & 44.7 & 33.6 & 0.0 & 0.3 & 5.0 & 84 & 0.484 \\
\hline 39PC482----- & 462 & 49.9 & 33.8 & 0.6 & 0.3 & & & 0.646 \\
\hline 39PC487----- & 467 & 45.8 & 31.0 & 0.4 & 0.3 & 4.6 & 82 & 0.718 \\
\hline 39PC492----- & 472 & 42.6 & 33.5 & 0.7 & 0.5 & & & 0.525 \\
\hline 39PC496----- & 476 & 42.6 & 22.4 & 1.3 & 0.3 & 4.8 & 71 & 0.478 \\
\hline 39PC502----- & 482 & 47.8 & 21.0 & 1.1 & 0.3 & & & 0.509 \\
\hline 39PC507----- & 487 & 40.6 & 21.3 & 0.8 & 0.4 & 4.6 & 68 & 0.406 \\
\hline 39PC512----- & 492 & 41.6 & 23.2 & 0.1 & 0.3 & & & 0.383 \\
\hline 39PC517----- & 497 & 35.4 & 27.9 & 1.5 & 0.3 & 4.7 & 70 & 0.383 \\
\hline 39PC522----- & 502 & 41.6 & 22.9 & 1.4 & 0.5 & & & 0.269 \\
\hline 39PC527----- & 507 & 46.8 & 24.3 & 0.7 & 0.4 & 7.7 & 80 & 0.345 \\
\hline 39PC532----- & 512 & 37.4 & 23.6 & 1.8 & 0.4 & & & 0.422 \\
\hline 39PC537----- & 517 & 37.4 & 28.6 & 1.5 & 0.3 & 8.2 & 76 & 0.356 \\
\hline 39PC542----- & 522 & 30.2 & 22.7 & 1.5 & 0.2 & & & 0.317 \\
\hline 39PC547----- & 527 & 35.4 & 17.5 & 1.6 & 0.4 & 8.8 & 64 & 0.343 \\
\hline 39PC552----- & 532 & 35.4 & 25.8 & 1.2 & 0.2 & & & 0.313 \\
\hline 39PC557----- & 537 & 20.8 & 28.6 & 1.9 & 0.4 & 6.6 & 58 & 0.345 \\
\hline 39PC562----- & 542 & 35.4 & 22.9 & 0.8 & 1.4 & & & 0.328 \\
\hline 39PC567----- & 547 & 36.4 & 31.0 & 0.7 & 0.3 & 7.6 & 76 & 0.291 \\
\hline 39PC572----- & 552 & 32.2 & 39.5 & 0.6 & 0.3 & & & 0.321 \\
\hline 39PC578----- & 558 & 42.6 & 31.6 & 0.0 & 0.3 & 8.0 & 83 & 0.357 \\
\hline 39PC582----- & 562 & 40.6 & 36.4 & 0.0 & 0.4 & & & 0.477 \\
\hline 39PC587----- & 567 & 32.2 & 36.2 & 0.0 & 0.6 & 8.4 & 77 & 0.479 \\
\hline 39PC592----- & 572 & 44.7 & 33.9 & 0.0 & 0.9 & & & 0.428 \\
\hline 39PC597----- & 577 & 48.9 & 34.1 & 0.0 & 0.4 & 10.0 & 93 & 0.477 \\
\hline 39PC602----- & 582 & 40.6 & 33.6 & 0.0 & 0.5 & & & 0.525 \\
\hline 39PC606----- & 586 & 37.4 & 39.6 & 0.0 & 0.4 & 8.9 & 86 & 0.484 \\
\hline 39PC612----- & 592 & 44.7 & 18.6 & 0.0 & 0.3 & & & 0.448 \\
\hline 39PC617----- & 597 & 39.5 & 44.9 & 0.0 & 0.3 & 9.4 & 94 & 0.461 \\
\hline 39PC622----- & 602 & 20.8 & 32.4 & 0.0 & 0.3 & & & 0.509 \\
\hline 39PC627----- & 607 & 43.7 & 32.4 & 0.0 & 0.5 & 7.9 & 84 & 0.574 \\
\hline 39PC632----- & 612 & 65.5 & 41.1 & 0.0 & 0.1 & & & 0.769 \\
\hline 39PC637----- & 617 & 64.5 & 38.3 & 0.0 & 0.2 & 9.6 & 113 & 1.001 \\
\hline 39PC642----- & 622 & 59.3 & 35.4 & 0.0 & 0.2 & & & 0.922 \\
\hline 39PC647----- & 627 & 59.3 & 35.4 & 0.0 & 0.2 & 3.4 & 98 & 0.844 \\
\hline 39PC652----- & 632 & 62.4 & 32.4 & 0.0 & 0.2 & & & 0.828 \\
\hline 39PC657----- & 637 & 62.4 & 35.3 & 0.0 & 0.2 & 3.5 & 101 & 0.881 \\
\hline 39PC662----- & 642 & 65.5 & 38.4 & 0.0 & 0.1 & & & 0.901 \\
\hline
\end{tabular}


Table 3. Major components in sediment samples from the Cariaco Basin, in percent, calculated from major-oxide concentrations (table 1)-Continued.

\begin{tabular}{|c|c|c|c|c|c|c|c|c|}
\hline Sample & $\begin{array}{c}\text { Depth } \\
(\mathrm{cm})\end{array}$ & $\begin{array}{c}\text { Siliciclastic } \\
\text { Debris }\end{array}$ & Calcite & Dolomite & Apatite & $\begin{array}{c}\text { Organic } \\
\text { Matter }\end{array}$ & Sum & $\begin{array}{l}\text { Density } \\
\left(\mathrm{g} \mathrm{cm}^{-3}\right) \\
\end{array}$ \\
\hline 39PC667----- & 647 & 63.4 & 30.9 & 0.0 & 0.2 & 2.8 & 97 & 0.921 \\
\hline 39PC672----- & 652 & 58.2 & 34.1 & 0.0 & 0.2 & & & 0.934 \\
\hline 39PC677----- & 657 & 68.6 & 26.2 & 0.0 & 0.1 & 2.8 & 98 & 0.941 \\
\hline 39PC682----- & 662 & 82.2 & 13.4 & 0.1 & 0.0 & & & 0.949 \\
\hline 39PC688----- & 667 & 78.0 & 17.6 & 0.2 & 0.0 & 2.8 & 99 & 1.008 \\
\hline 39PC692----- & 672 & 81.1 & 13.2 & 0.9 & 0.0 & & & 0.984 \\
\hline 39PC697----- & 677 & 92.6 & 3.7 & 1.3 & -0.1 & 2.2 & 100 & 0.951 \\
\hline 39PC702----- & 682 & 95.7 & -0.1 & 1.1 & -0.2 & & & 0.997 \\
\hline 39PC706----- & 686 & 68.6 & 20.3 & 1.2 & 0.1 & 1.5 & 92 & 0.997 \\
\hline 39PC712----- & 692 & 78.0 & 13.5 & 1.1 & 0.0 & & & 0.958 \\
\hline 39PC717----- & 697 & 82.2 & 9.3 & 1.7 & 0.0 & 2.1 & 95 & 0.981 \\
\hline 39PC722----- & 702 & 99.8 & 1.1 & 1.3 & -0.2 & & & 0.961 \\
\hline 39PC727----- & 707 & 45.8 & 18.7 & 24.6 & 0.3 & 1.4 & 91 & 0.988 \\
\hline 39PC732----- & 712 & 52.0 & 14.9 & 26.5 & 0.3 & & & 1.368 \\
\hline 39PC737----- & 717 & 43.7 & 16.4 & 24.6 & 0.3 & 0.9 & 86 & 1.074 \\
\hline 39PC742----- & 722 & 48.9 & 20.1 & 17.3 & 0.2 & & & 0.972 \\
\hline 39PC747----- & 727 & 55.1 & 21.3 & 5.1 & 0.1 & 2.1 & 84 & 1.038 \\
\hline 39PC752----- & 732 & 57.2 & 18.1 & 2.6 & 0.1 & & & 0.897 \\
\hline 39PC757----- & 737 & 51.0 & 16.8 & 1.2 & 0.1 & 2.4 & 71 & 0.845 \\
\hline 39PC762----- & 742 & 49.9 & 16.0 & 1.4 & 0.1 & & & 0.984 \\
\hline 39PC767----- & 747 & 49.9 & 20.6 & 0.4 & 0.2 & 2.6 & 74 & 1.066 \\
\hline 39PC772----- & 752 & 63.4 & 23.1 & 1.0 & 0.1 & & & 1.051 \\
\hline 39PC777----- & 757 & 49.9 & 38.9 & 0.2 & 0.2 & 2.4 & 92 & 0.928 \\
\hline 39PC782----- & 762 & 51.0 & 31.0 & 1.1 & 0.2 & & & 0.768 \\
\hline 39PC787----- & 767 & 48.9 & 38.0 & 2.0 & 0.2 & 2.2 & 91 & 0.799 \\
\hline 39PC792----- & 772 & 51.0 & 37.5 & 2.7 & 0.2 & & & 0.787 \\
\hline 39PC797----- & 777 & 51.0 & 34.3 & 4.1 & 0.2 & 4.4 & 94 & 0.821 \\
\hline 39PC802----- & 782 & 46.8 & 39.4 & 4.1 & 0.2 & & & 0.795 \\
\hline 39PC807----- & 787 & 47.8 & 38.9 & 4.9 & 0.3 & 4.2 & 96 & 0.738 \\
\hline 39PC812----- & 792 & 45.8 & 37.4 & 7.7 & 0.5 & & & 0.741 \\
\hline 39PC817----- & 797 & 43.7 & 41.2 & 5.5 & 0.4 & 4.4 & 95 & 0.755 \\
\hline 39PC822----- & 802 & 48.9 & 33.3 & 6.0 & 0.3 & & & 0.756 \\
\hline 39PC827----- & 807 & 44.7 & 38.0 & 6.9 & 0.3 & 4.2 & 94 & 0.744 \\
\hline 39PC832----- & 812 & 43.7 & 38.0 & 7.0 & 0.4 & & & 0.771 \\
\hline 39PC837----- & 817 & 45.8 & 38.4 & 5.9 & 0.3 & 4.4 & 95 & 0.793 \\
\hline 39PC842----- & 822 & 45.8 & 39.8 & 3.4 & 0.3 & & & 0.791 \\
\hline 39PC847----- & 827 & 45.8 & 37.0 & 4.0 & 0.3 & 4.3 & 91 & 0.781 \\
\hline 39PC852----- & 832 & 48.9 & 37.9 & 2.0 & 0.3 & & & 0.745 \\
\hline 39PC857----- & 837 & 46.8 & 40.6 & 1.6 & 0.4 & 5.6 & 95 & 0.781 \\
\hline 39PC862----- & 842 & 47.8 & 41.0 & 0.6 & 0.4 & & & 0.791 \\
\hline 39PC867----- & 847 & 48.9 & 38.3 & 1.2 & 0.4 & 6.5 & 95 & 0.789 \\
\hline 39PC872----- & 852 & 52.0 & 35.5 & 1.5 & 0.3 & & & 0.789 \\
\hline 39PC877----- & 857 & 48.9 & 38.8 & 0.3 & 0.3 & 6.8 & 95 & 0.795 \\
\hline
\end{tabular}


Table 3. Major components in sediment samples from the Cariaco Basin, in percent, calculated from major-oxide concentrations (table 1)-Continued.

\begin{tabular}{ccccccccr}
\hline Sample & $\begin{array}{c}\text { Depth } \\
(\mathrm{cm})\end{array}$ & $\begin{array}{c}\text { Siliciclastic } \\
\text { Debris }\end{array}$ & Calcite & Dolomite & Apatite & $\begin{array}{c}\text { Organic } \\
\text { Matter }\end{array}$ & Sum & $\begin{array}{c}\text { Density } \\
\left(\mathrm{g} \mathrm{cm}^{-3}\right)\end{array}$ \\
\hline 39PC882----- & 862 & 46.8 & 41.1 & 0.7 & 0.4 & & & 0.823 \\
39PC887---- & 867 & 47.8 & 38.3 & 1.2 & 0.4 & 6.1 & 94 & 0.751 \\
39PC892---- & 872 & 46.8 & 41.1 & 0.7 & 0.3 & & & 0.735 \\
39PC897----- & 877 & 46.8 & 41.1 & 0.7 & 0.4 & 6.7 & 96 & 0.761 \\
39PC902----- & 882 & 47.8 & 41.6 & 0.0 & 0.3 & & & 0.751 \\
39PC907---- & 887 & 46.8 & 41.5 & 0.0 & 0.4 & 6.5 & 95 & 0.768 \\
39PC912---- & 892 & 45.8 & 41.1 & 0.8 & 0.3 & & & 0.789 \\
39PC917---- & 897 & 45.8 & 41.2 & 0.8 & 0.3 & 5.9 & 94 & 0.776 \\
39PC922----- & 902 & 45.8 & 41.1 & 0.8 & 0.3 & & & 0.791 \\
39PC927----- & 907 & 46.8 & 41.1 & 0.7 & 0.3 & 6.0 & 95 & 0.802 \\
39PC932---- & 912 & 46.8 & 41.6 & 0.0 & 0.3 & & & 0.778 \\
39PC937---- & 917 & 44.7 & 44.0 & 0.2 & 0.3 & 6.1 & 95 & 0.785 \\
39PC942----- & 922 & 45.8 & 40.7 & 1.6 & 0.3 & & & 0.808 \\
39PC947----- & 927 & 42.6 & 44.4 & 0.0 & 0.4 & 6.2 & 94 & 0.818 \\
39PC952---- & 932 & 48.9 & 41.2 & 0.5 & 0.3 & & & 0.824 \\
39PC957---- & 937 & 46.8 & 41.2 & 0.7 & 0.3 & 6.2 & 95 & 0.812 \\
39PC962----- & 942 & 45.8 & 44.4 & 0.0 & 0.3 & & & 0.796 \\
39PC967----- & 947 & 45.8 & 41.2 & 0.8 & 0.3 & 6.0 & 94 & 0.778 \\
39PC972---- & 952 & 42.6 & 44.4 & 0.0 & 0.4 & & & 0.799 \\
39PC977---- & 957 & 38.5 & 52.6 & 0.0 & 0.3 & 6.2 & 98 & 0.806 \\
39PC982---- & 962 & 46.8 & 41.2 & 0.7 & 0.3 & & & 0.813 \\
39PC986----- & 966 & 45.8 & 40.7 & 1.6 & 0.3 & & & \\
39PC992----- & 972 & 44.7 & 40.3 & 2.6 & 0.3 & & & \\
\hline
\end{tabular}

${ }^{1}$ A complete discussion on calculating each component from bulk chemical analyses is given in Piper and Isaacs (1994, 1996). The calculations of the terrigenous fraction and calcite are given in this paper and the supporting data presented in figure 4 and discussed in the text. The ratio of organic matter to organic-carbon for plankton is 2.8. The ratio for ancient sedimentary rocks is as low as 1.5 (Powell and others, 1975). An arbitrarily taken single value of two is taken for the ratio of organic matter to organic carbon in sediment from core PL07-39PC, to improve the sum values, which then allow biogenic silica to be estimated by difference to 100 percent (not shown). Biogenic silca is consistantly present in the depth interval between 300 and $600 \mathrm{~cm}$. Blanks indicate no data are available.

Table 4. Correlation coefficients for major-element oxides in sediment from the Cariaco Basin.

[Org. C, organic carbon; and sed. rate, sedimentation rate]

\begin{tabular}{lcccccccc}
\hline & org. $\mathrm{C}$ & $\begin{array}{c}\text { sed. } \\
\text { rate }\end{array}$ & $\mathrm{Al}_{2} \mathrm{O}_{3}$ & $\mathrm{TiO}_{2}$ & $\mathrm{~K}_{2} \mathrm{O}$ & $\mathrm{Fe}_{2} \mathrm{O}_{3}$ & $\mathrm{CaO}$ & $\mathrm{MgO}$ \\
\hline org. C------ & 1 & & & & & & & \\
sed. rate--- & -.795 & 1 & & & & & & \\
$\mathrm{Al}_{2} \mathrm{O}_{3}-------$ & .005 & .028 & 1 & & & & & \\
$\mathrm{TiO}_{2}-------$ & -.233 & .215 & .947 & 1 & & & & \\
$\mathrm{~K}_{2} \mathrm{O}--------$ & .066 & .079 & .962 & .871 & 1 & & & \\
$\mathrm{Fe}_{2} \mathrm{O}_{3}-------$ & .028 & .030 & .989 & .928 & .966 & 1 & & \\
$\mathrm{CaO}^{--------}$ & -.186 & .018 & -.358 & -.304 & -.406 & -.400 & 1 & \\
$\mathrm{MgO-------}$ & -.516 & .385 & -.029 & .079 & -.059 & -.064 & .530 & 1 \\
\hline
\end{tabular}



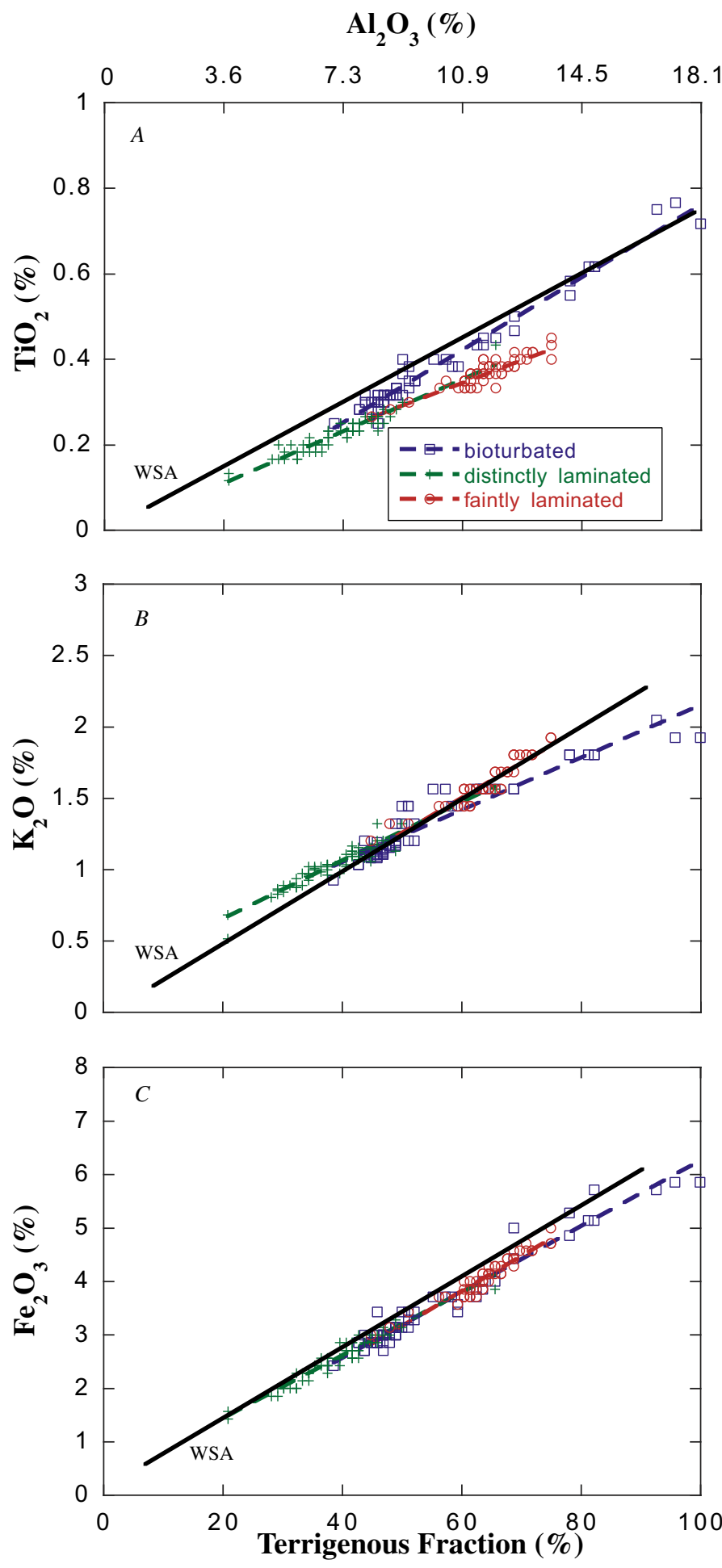

Figure 5. Relations between the concentration of the terrigenous fraction (and $\mathrm{Al}_{2} \mathrm{O}_{3}$ taken from figure 4) and the concentrations of selected major-element oxides in sediment from core PL07-39PC. The broken curves give the linear regression curves for each element oxide for the three different lithologies of the core (fig. 2). The solid curves give the relations for the world shale average (WSA) (Wedepohl, 1969-1978; Piper and Isaacs, 1996), mixed with diluting phases, for example biogenic $\mathrm{SiO}_{2}$, calcite-aragonite, and organic matter. The regression curves for a single major-element oxide commonly cannot be distinguished; they also may be masked by the curve representing the relation in WSA. will little affect the calculation of their marine contribution. A Mo concentration in the terrigenous fraction, nonetheless, might be obtained by a curve drawn through those samples with the lowest measured concentrations and its extrapolation to 100 percent terrigenous material. The curve is subsequently referred to as the terrigenous maximum. The curve extrapolates to the WSA value for Mo of $2.0 \mathrm{ppm}$ (table 2), supporting the interpretation that the curve defines the terrigenous contribution of Mo. The concentration of $\mathrm{Cd}$ in samples from the bioturbated section of the core is commonly below the detection limit of $2 \mathrm{ppm}$ (table 1). A Cd concentration of $0.2 \mathrm{ppm}$ in the terrigenous fraction, its established concentration in WSA, is assumed and assigned to samples with measured $\mathrm{Cd}$ values below the detection limit. Clearly, some of these samples may have a $\mathrm{Cd}$ concentration between a terrigenous contribution and its limit of detection.

A large marine source for $\mathrm{Cd}$ and Mo is supported by their high factor loading on the factor dominated by organic matter (table 5). Such loadings, or correlations, may be further indicative of the phase within the sediment that hosts a trace element.

Trace elements that constitute a third group include $\mathrm{Cr}$, $\mathrm{Cu}, \mathrm{Ni}, \mathrm{Pb}, \mathrm{V}$, and Zn (fig. 9). Similar to the trace elements in the first group, they have high factor loadings on the factor for which the terrigenous fraction has a high factor loading (table 5). Unlike the first group, a single regression curve for each trace element extrapolates to a positive value on the $\mathrm{y}$-axis and to a value greater than the WSA value at 100 per cent terrigenous fraction (table 2). One method of estimating the terrigenous contribution of this group is to assume WSA concentrations. The terrigenous maximum for Mo (fig. 8A) and regression curve for trace elements interpreted as having only a terrigenous contribution (for example, $\mathrm{Co}, \mathrm{Ga}$, the rareearth elements, and Th) support this procedure. However, even the distributions of these elements (fig. 6) suggested slightly different concentrations in the terrigenous fraction of the different lithologic units, similar to $\mathrm{K}_{2} \mathrm{O}$ and $\mathrm{TiO}_{2}$ (Yarincik and others, 2000). As variations in contributions by the several sources of the terrigenous fraction during the last $20 \mathrm{kyr}$ were quite prominent (Yarinick and others, 2000), a terrigenous fraction of variable composition might have been expected.

These apparent variations in composition of the terrigenous fraction clearly place an uncertainty on the calculations of the seawater contribution of trace elements made in this study, particularly for this third group, as the terrigenous contribution dominants the trace-element inventory. For example, $\mathrm{Pb}$ values in the bioturbated samples define rather poorly a composition equal to a WSA value that allows for only a minor marine fraction. $\mathrm{Cr}, \mathrm{Ni}$, and $\mathrm{V}$ values in samples from the bioturbated section also seem to define a terrigenous maximum, but the curves extrapolate to concentrations at 100 percent terrigenous debris that are greater than their concentrations in WSA (table 2). These trends suggest that the composition of the bioturbated sediment section might be representative of the terrigenous fraction, except for $\mathrm{Cu} . \mathrm{Cu}$ has its highest concentration in samples from the bioturbated section. Zn shows a single trend, which gives an extrapolated 

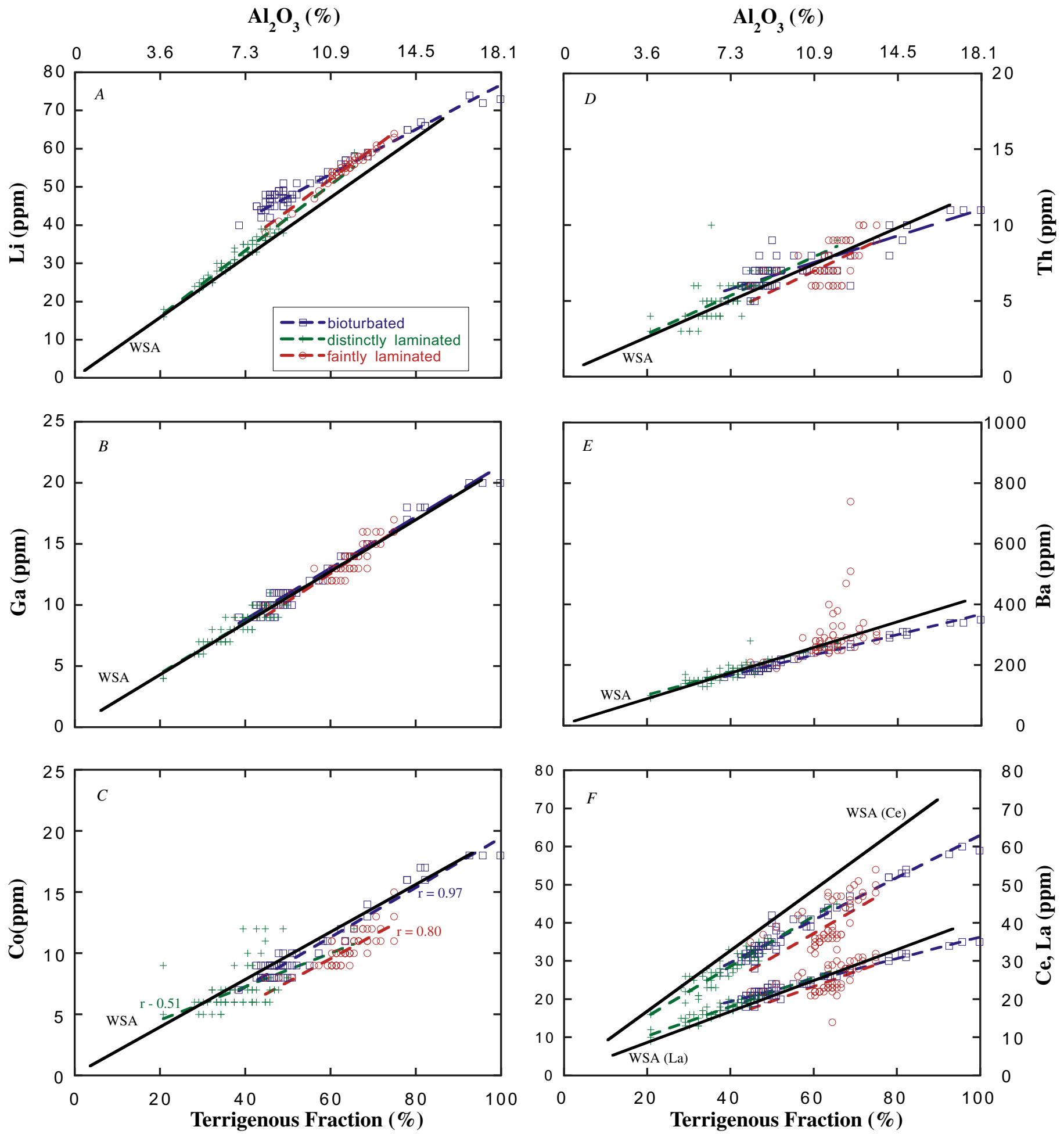

Figure 6. Relations between the concentration of the terrigenous fraction and the concentrations of trace elements that exhibit approximate single trends with the terrigenous fraction from the three sediment lithologies of core PL07-39PC. Symbols and abbreviations are the same as in figure 5. The curves for each trace element extrapolate to the origin and to approximate concentrations in the world shale average (WSA) at 100 percent terrigenous debris. The Ba concentration in samples between 165 and $200-\mathrm{cmbsf}$ (table 1 ) is above a solely terrigenous contribution. The relation between terrigenous debris and $\mathrm{Al}_{2} \mathrm{O}_{3}$ is taken from figure 4 . 
Table 5. Varimax orthogonal transformation solution, principal components factor extraction method, of the log10 of trace-element analyses and major sediment components in sediment core PL07-39PC, calculated from major-element-oxide analyses, using Statview ${ }^{\text {tm }}$ II software.

[Values greater than 0.5 are in bold. Org. mat., organic matter]

\begin{tabular}{|c|c|c|c|c|}
\hline & Factor 1 & Factor 2 & Factor 3 & Factor 4 \\
\hline Siliciclastics---- & 0.96 & -0.16 & -0.08 & -0.12 \\
\hline Calcite---------- & 0.11 & -0.02 & 0.97 & -0.06 \\
\hline Dolomite------- & -0.13 & -0.40 & 0.02 & 0.78 \\
\hline Apatite---------- & -0.41 & 0.66 & 0.17 & 0.16 \\
\hline Org. mat.------ & -0.06 & 0.89 & 0.03 & -0.27 \\
\hline Ba-------------- & 0.87 & -0.20 & -0.12 & -0.22 \\
\hline Cd-------------- & -0.31 & 0.62 & -0.57 & -0.18 \\
\hline Ce------------- & 0.89 & -0.25 & -0.01 & -0.18 \\
\hline Co------------- & 0.89 & -0.16 & -0.04 & 0.23 \\
\hline Cr------------ & 0.92 & 0.18 & 0.03 & 0.10 \\
\hline Cu------------ & 0.86 & -0.02 & 0.30 & 0.31 \\
\hline Ga-------------- & 0.96 & -0.14 & -0.04 & -0.08 \\
\hline La------------- & 0.93 & -0.19 & 0.07 & -0.17 \\
\hline Li-------------- & 0.95 & -0.17 & 0.16 & 0.00 \\
\hline Mo------------- & -0.42 & 0.82 & -0.12 & -0.28 \\
\hline Nd-------------- & 0.80 & -0.31 & 0.38 & -0.14 \\
\hline Ni------------ & 0.79 & 0.38 & -0.11 & 0.27 \\
\hline Pb-------------- & 0.52 & -0.21 & -0.66 & -0.22 \\
\hline Sr------------ & 0.06 & -0.09 & 0.97 & -0.02 \\
\hline Th------------- & 0.73 & -0.15 & -0.12 & -0.12 \\
\hline V--------------- & 0.55 & 0.68 & -0.30 & 0.11 \\
\hline Y--------------- & 0.77 & -0.27 & 0.51 & 0.10 \\
\hline Zn------------- & 0.97 & 0.00 & 0.16 & 0.04 \\
\hline
\end{tabular}

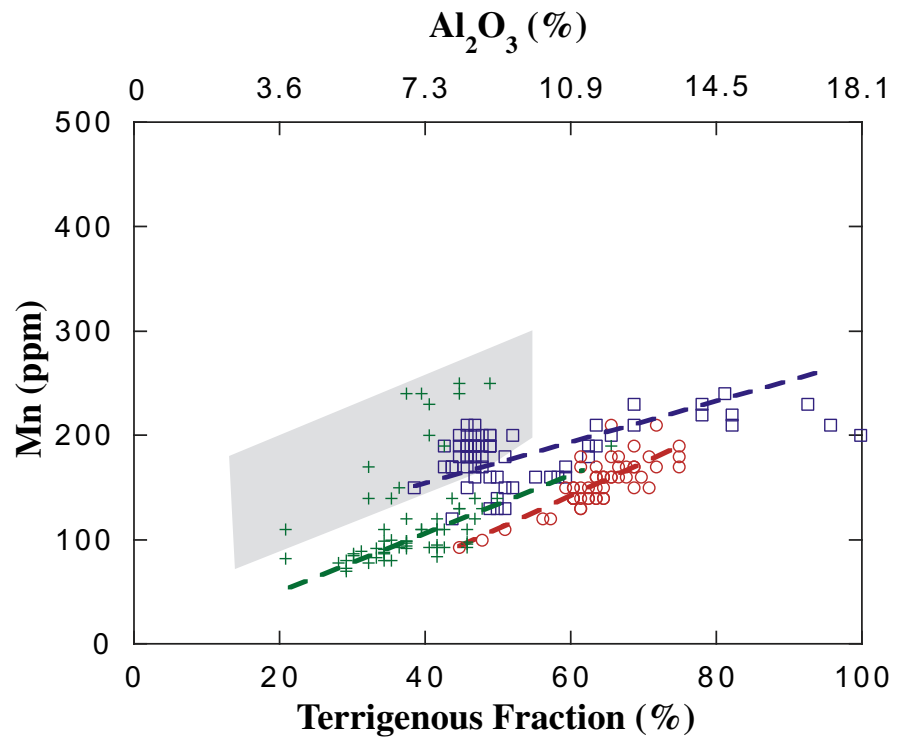

Figure 7. The relation between the concentrations of the terrigenous fraction and manganese $(\mathrm{Mn})$ in sediment of core PL07-39PC. Samples within the shaded area are from the depth intervals 530 to 590 cmbsf (centimeters below the sea floor) and 760 to $900 \mathrm{cmbsf}$. Symbols representing the samples are the same as in figure 5 . The relation between $\mathrm{Al}_{2} \mathrm{O}_{3}$ and terrigenous debris is taken from figure 4. 

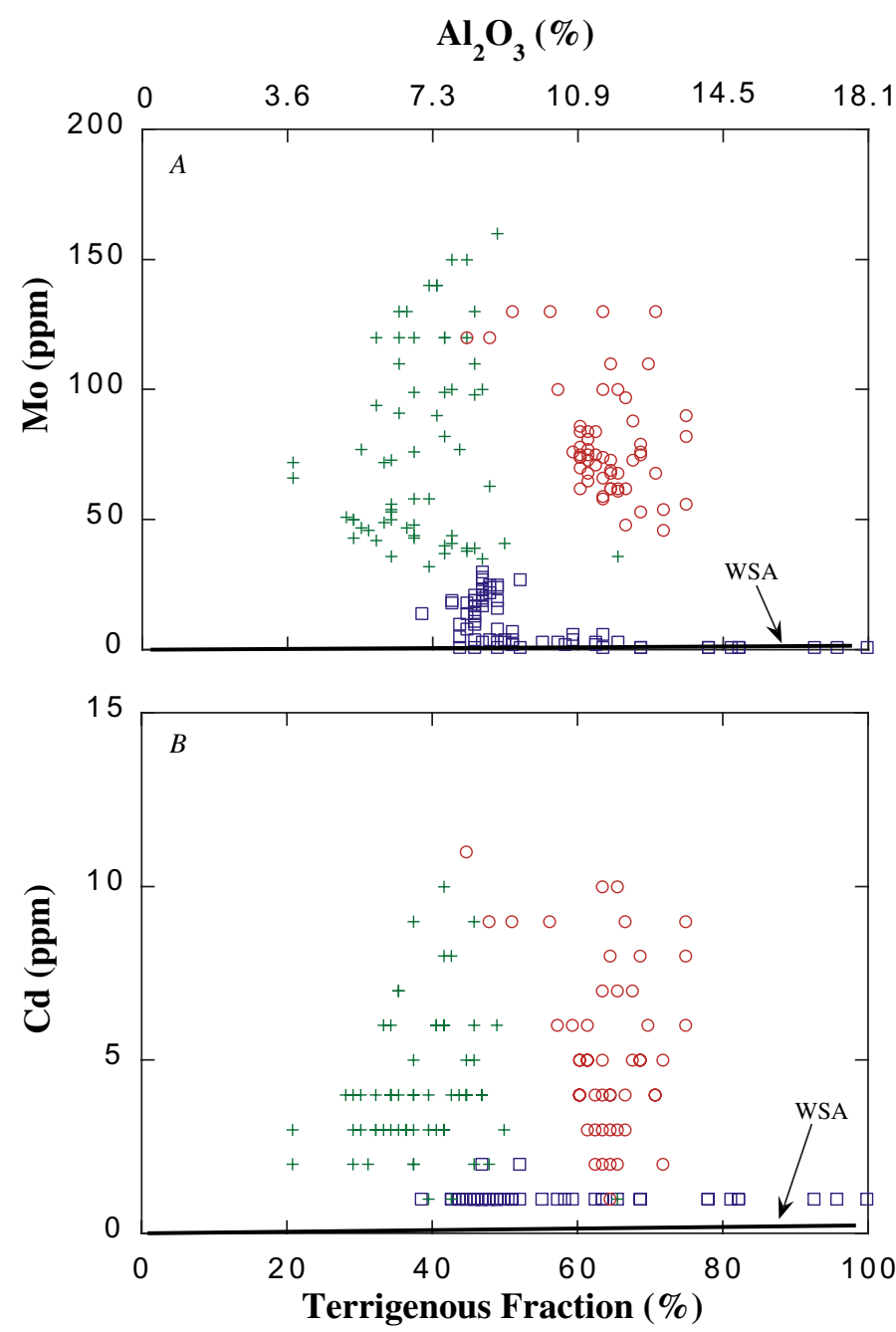

Figure 8. The relations between the concentration of the terrigenous fraction and the concentrations of $\operatorname{Mo}(A)$ and $\mathrm{Cd}(B)$ in sediment of core PL07-39PC. In $A$, a single curve defines the minimum values and the Mo concentration in the world shale average (WSA). In $B$, the curve for WSA represents a $\mathrm{Cd}$ concentration of $0.2 \mathrm{ppm}$, although the detection limit for $\mathrm{Cd}$ by ICP-AES is $2 \mathrm{ppm}$. Samples having a concentration less than $2 \mathrm{ppm}$ are plotted as having a concentration of 1 ppm, but they could have an even lower concentration. Symbols and abbreviations are the same as in figure 5 . The relation between $\mathrm{Al}_{2} \mathrm{O}_{3}$ and terrigenous debris is taken from figure 4.

value that is significantly greater than its concentration in WSA (fig. 9).

Two different criteria are used to estimate the terrigenous contribution of this third group of trace elements, recognizing that any procedure employed might introduce possible errors. For $\mathrm{Cr}, \mathrm{Ni}, \mathrm{Pb}$, and $\mathrm{V}$, the terrigenous contribution is defined by samples from the bioturbated section of the core with lowest concentrations. For $\mathrm{Cu}$ and $\mathrm{Zn}$, the terrigenous contribution is based on concentrations of $\mathrm{Cu}$ and $\mathrm{Zn}$ in WSA (fig. 9; table 2). The procedure gives a major seawater contribution of $\mathrm{Cu}$ and $\mathrm{Zn}$ to the bioturbated section of the core. This contribution may represent an artifact of an incorrectly adjusted siliciclastic contribution of $\mathrm{Cu}$ and $\mathrm{Zn}$ and not a high seawater input. However, using the same procedure that was used to determine the terrigenous contributions of $\mathrm{Cr}, \mathrm{Ni}, \mathrm{Pb}$, and $\mathrm{V}$ would give negative concentrations of marine $\mathrm{Cu}$ and $\mathrm{Zn}$ in many samples from the laminated sections of the core. Thus, trace-element distributions below $730 \mathrm{cmbsf}$, or prior to $17 \mathrm{ka}$, are considered only very briefly. The trace-element compositions of these different terrigenous sources need to be ascertained by some alternative method, possibly through a leaching experiment, before a more complete discussion can be presented.

The trace-element fraction in each sample above the terrigenous contribution is partitioned into the two marine source fractions-(1) the biogenic fraction, composed mostly of planktonic debris derived from the photic zone (Schuffert and others, 1994; Thunell and others, 2000) and (2) the hydrogenous fraction, derived from seawater by precipitation and adsorption reactions (Goldberg, 1963). Similar to the terrigenous fraction, the two marine sources may not have had constant compositions throughout the depositional history of the core. Based on an analysis of cores collected by the Ocean Drilling Program, the actual species composition of the algal community shows a change in the main primary producers at about $9.5 \mathrm{ka}$, from dominantly diatoms to dominantly nannoplankton (Werne and others, 2000). The same change is seen in core PL07-39PC at about $270 \mathrm{cmbsf}$ (10 ka; fig. 2). The estimated concentration of biogenic $\mathrm{SiO}_{2}$ decreases from as great as 30 percent to less than 5 percent (table 3; Peterson and others, 1995). The trace-nutrient demand of the two algal communities may have been quite different. Unfortunately, it is not currently possible to evaluate this uncertainty. In the calculations presented here, their overall trace-nutrient demands are assumed to have been the same.

The depositional rates of $\mathrm{Cd}, \mathrm{Cu}, \mathrm{Ni}$, and $\mathrm{Zn}$ offer perhaps the best opportunity to ascertain the trace-element contribution by organic matter (table 6). All four are taken up in the photic zone by plankton (Martin and Knauer, 1973; Boyle and others, 1976, Boyle and others, 1977; Sclater and others, 1976; Collier and Edmond, 1984; Brumsack, 1986). The stoichiometry for organic matter, that is, its elemental composition (table 2), can be taken from recent analyses of plankton and suspended organic matter collected from the Pacific Ocean (Martin and Knauer, 1973; Collier and Edmonds, 1984; Sherrell, 1989). Although these measurements exhibit considerable scatter (Piper, 1994), the best estimate from the measurements for individual trace elements has been examined by comparing the measured value with the value calculated from its seawater profile (Piper and Isaacs, 1994, 1996). This procedure is similar to evaluating the nitrogen and phosphorus stoichiometry of organic matter (Redfield and others, 1963). For the open ocean, agreement is quite good, particularly for $\mathrm{Cd}$ and $\mathrm{Cu}$.

This scheme is used to calculate the $\mathrm{Cd}, \mathrm{Cu}$, and $\mathrm{Ni}$ concentrations in plankton within the area of the Cariaco Basin. The seawater-depth profiles of the three trace elements in the Venezuela Basin of the Caribbean Sea (Jacobs and others, 1987) are used rather than their seawater profiles in the open ocean. The method relies on the profiles being determined by the uptake of trace elements in the photic zone by algae, oxidation of the host organic matter by bacteria and zooplankton 


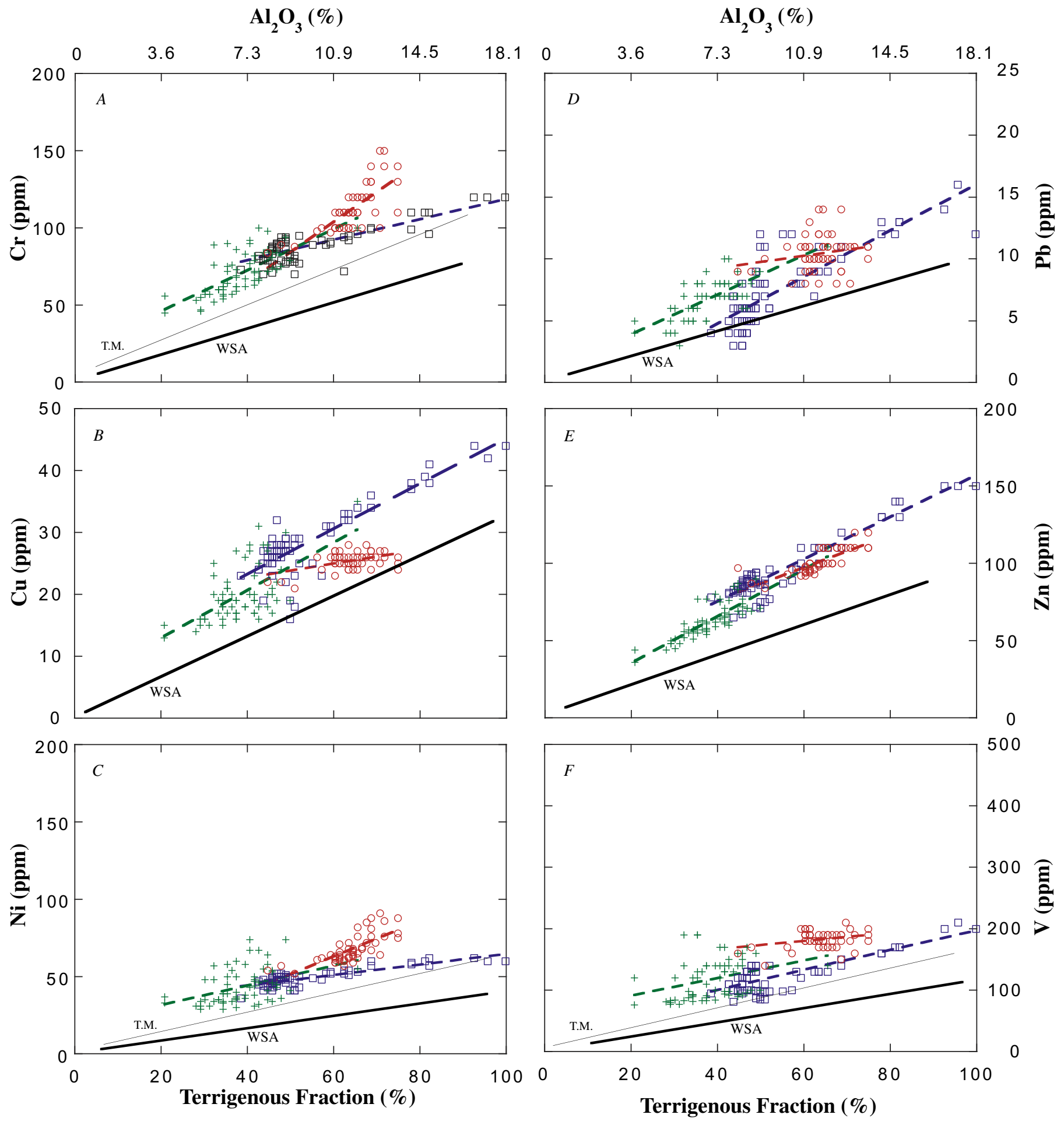

Figure 9. The relations between the concentration of the terrigenous fraction and the concentrations of trace elements in sediment of core PL07-39PC that have strong factor loadings on the same factor as $\mathrm{Al}_{2} \mathrm{O}_{3}$ (table 5 ) but have concentrations (table 1) that are significantly greater than can be attributed solely to a terrigenous contribution (table 2). Least-square best-fit curves for this group of elements commonly extrapolate to positive values on the y-axis. The curves labeled T.M. (terrigenous maximum) give the concentrations of $\mathrm{Cr}$, $\mathrm{Ni}$, $\mathrm{Pb}$, and $\mathrm{V}$ in the terrigenous fraction, as determined from their minimum values, similar to the determination of Mo in the terrigenous fraction (fig. $8 A$ ). Symbols and abbreviations are the same as in figure 5 . The relation between $\mathrm{Al}_{2} \mathrm{O}_{3}$ and the terrigenous fraction is taken from figure 4 . 
Table 6. Table 6. Concentrations of trace elements in seawater and organic matter in the Cariaco Basin and their rates of deposition in the surface sediment of core PL07-39PC.

[Calculated rates are based on trace-element concentrations in organic matter (the biogenic contribution) and terrigenous debris (the terrigenous contribution) and the flux of each through the water column and on the bottom-water residence time and difference in elemental concentrations in source water and bottom water (the hydrogenous contribution). Measured rates represent accumulation rates based on the concentrations of trace elements in the surface sediment and the bulk sediment accumulation rate]

\begin{tabular}{|c|c|c|c|c|c|c|c|c|}
\hline Metal & $\begin{array}{l}\text { Cariaco Basin } \\
\text { Source Water } \\
\qquad(p p b)^{1}\end{array}$ & $\begin{array}{l}\text { Cariaco Basin } \\
\text { Bottom Water } \\
\qquad(p p b)^{1}\end{array}$ & $\begin{array}{c}\text { Concentration in } \\
\text { Organic Matter } \\
(\mathrm{ppm})^{2}\end{array}$ & $\begin{array}{c}\text { Terrigenous } \\
\text { Contribution }(\mu \mathrm{g} \\
\left.\mathrm{cm}^{-2} \mathrm{yr}^{-1}\right)^{3}\end{array}$ & $\begin{array}{c}\text { Biogenic } \\
\text { Contribution } \\
\left(\mu \mathrm{g} \mathrm{cm}^{-2} \mathrm{yr}^{-1}\right)^{3}\end{array}$ & $\begin{array}{l}\text { Hydrogenous } \\
\text { Contribution } \\
\left(\mu \mathrm{g} \mathrm{cm}^{-2} \mathrm{yr}^{-1}\right)^{3}\end{array}$ & $\begin{array}{c}\text { Calculated } \\
\text { Rate } \\
\left(\mu \mathrm{g} \mathrm{cm}^{-2} \mathrm{yr}^{-1}\right)\end{array}$ & $\begin{array}{c}\text { Measured } \\
\text { Rate } \\
\left(\mu \mathrm{g} \mathrm{cm}^{-2} \mathrm{yr}^{-1}\right)\end{array}$ \\
\hline Cd----- & 0.012 & 0.001 & 5.5 & 0.002 & 0.049 & 0.006 & 0.057 & 0.061 \\
\hline Cu------ & 0.069 & 0.028 & 7.5 & 0.243 & 0.067 & 0.029 & 0.339 & 0.318 \\
\hline Zn------ & 0.124 & 0.043 & $110(30)^{4}$ & 0.736 & $0.978(0.267)^{4}$ & 0.049 & $1.763(1.052)^{4}$ & 1.187 \\
\hline Cr------ & 0.22 & 0.088 & 2 & 0.883 & 0.018 & 0.053 & 0.954 & 1.224 \\
\hline
\end{tabular}

${ }^{1}$ Concentrations of $\mathrm{Mo}, \mathrm{Cd}, \mathrm{Cu}, \mathrm{V}$, and $\mathrm{Ni}$ in source water are values of water in the Cariaco basin between 100- and 250-m depth (Jacobs and others, 1987; Emerson and Huested, 1991). Concentrations of $\mathrm{Zn}$ and $\mathrm{Cr}$ in source water, in itilics, are of open-ocean seawater in the North Atlantic (Bruland and Franks, 1983) and North Pacific (Murray and others, 1983) Oceans, respectively. Concentrations of metals in bottom water are from Jacobs and others (1987), except for $\mathrm{Zn}$ and $\mathrm{Cr}$. Their bottom-water concentrations assume 65 percent and 60 percent removal, respectively, from source water via precipitation and adsorption reactions. These estimates are based on concentration profiles of $\mathrm{Zn}$ in other basins (fig. 10A ) and $\mathrm{Cr}$ profiles in the water column in Saanich Inlet (Emerson and others, 1979), in which approximately 60 percent of the $\mathrm{Cr}(\mathrm{VI})$ in oxic water is reduced at depth to $\mathrm{Cr}(\mathrm{III})$.

${ }^{2}$ The $\mathrm{Cd}, \mathrm{Cu}$ and Ni concentrations in organic matter are based on their depth profiles and that of phosphate in the Caribbean Sea (Jacobs and others, 1987) and the phosphate concentration in organic matter (Redfield and others, 1963). The Ni value of 18 ppm is discussed in the text. The $\mathrm{Cu}$ concentration is, at best, an approximation. The Mo value represents a maximum, based on its depth profile and the precision of the Mo analyses. The $\mathrm{Cr}, \mathrm{V}$, and $\mathrm{Zn}$ values represent published measurements (see Piper, 1994, for sources of data).

${ }^{3}$ The current accumulation rate of terrigenous sediment is $7.36 \mathrm{mg} \mathrm{cm}^{-2} \mathrm{yr}^{-1}$; the flux of organic matter into the bottom water is $8.89 \mathrm{mg}$ $\mathrm{cm}^{-2} \mathrm{yr}^{-1}$; the volume of bottom water above the core site is $60 \mathrm{~L} \mathrm{~cm}^{-2}$, or $600 \mathrm{~m}$; and the residence time of bottom water is $100 \mathrm{yr}$.

${ }^{4}$ The values in parentheses are based on a $\mathrm{Zn}$ concentration in algal debris of $30 \mathrm{ppm}$. See text for explanation.

at depth, and advection. In support of this assumption, the $\mathrm{Cd}: \mathrm{PO}_{4}{ }^{3-}$ ratio in the water column is constant in the Venezuela Basin (Jacobs and others, 1987), although its value of $0.6 \times 10^{-3}$ is slightly less than half the stoichiometric value of organic matter from the Pacific Ocean (Brumsack, 1986). The strongly varying absolute concentrations of both $\mathrm{Cd}$ and $\mathrm{PO}_{4}{ }^{3-}$, but constant $\mathrm{Cd}: \mathrm{PO}_{4}{ }^{3-}$ ratio, further suggests that the trace-element stoichiometry of particulate organic matter is maintained throughout the water column and onto the sea floor. The $\mathrm{Cu}$ value also is significantly less than its value in organic debris and plankton from the Pacific Ocean, but its profile is rather complex; the Ni value is 2.4-fold greater than its measured value in plankton (table 2). The value for $\mathrm{Ni}$ is likely too high as few analyses of plankton and organic detritus have recorded such a high value (18 ppm). However, this value is used as a measure of the export of $\mathrm{Ni}$ from the photic zone into the bottom water, recognizing that the actual uptake of Ni by plankton as a trace nutrient is likely less than this value.

The contribution of trace elements by organic matter can be calculated from the stoichiometry of the organic matter (table 6) and the rain rate of organic matter into the bottom water. Sediment traps suspended in the water column (MüllerKarger and others, 2001) have shown that only about 1.7 percent of the organic matter produced in the photic zone survives to the sea floor (Thunell and others, 2000; MüllerKarger and others, 2001). Thunell and others (2000) have demonstrated that oxidation of organic matter occurs largely above $250 \mathrm{~m}$, but it continues throughout the deeper part of the water column. Because of the method used here of calculating the contribution of trace elements by the bottom water (the hydrogenous fraction), the trace-element fraction within the organic matter that is oxidized within the $\mathrm{SO}_{4}{ }^{2-}$-reducing region of the water column must be added to the fraction that actually settles onto the seafloor as particulate organic matter. For example, the profile of $\mathrm{Cd}$ in the Cariaco Basin (Jacobs and others, 1987) suggests that 90 percent of the Cd advecting into the bottom water is removed by precipitation/adsorption reactions, the hydrogenous fraction. This amount is in addition to the amount released in the water column via oxidation of settling organic matter. Thus, the biogenic input of trace elements to the sediment is defined by the organic matter settling into the uppermost layer of the $\mathrm{SO}_{4}{ }^{2-}$-reducing region of the water column, not merely by the much smaller fraction of organic matter that settles onto the sea floor. The organic matter collected by the sediment trap suspended in the water column at 275-m depth (Müller-Karger and others, 2001) is assumed to have measured the flux of organic matter settling into the bottom water.

The hydrogenous fraction that accumulates under each of the different levels of bacterial respiration in bottom water is characterized by a unique suite of trace elements (Piper and Isaacs, 1994, 1996). As examples, Co and Mn accumulate within the hydrogenous fraction under bottom-water condi- 
tions of $\mathrm{O}_{2}$ respiration. The rare-earth elements accumulate under conditions of $\mathrm{O}_{2}$ respiration and denitrification. $\mathrm{Cr}$ and $\mathrm{V}$ accumulate under conditions of denitrification and $\mathrm{SO}_{4}{ }^{2-}$ reduction. Mo accumulates only under the more negative redox conditions of $\mathrm{SO}_{4}^{2-}$ reduction. The marine fraction of each of these trace elements should be dominated by their hydrogenous fraction, as all have low concentrations in organic debris (table 2).

Mo also has a low concentration in terrigenous debris (table 2), making it an ideal trace element for identifying conditions of $\mathrm{SO}_{4}{ }^{2-}$ reduction in the bottom water. $\mathrm{Cd}, \mathrm{Cu}, \mathrm{V}$, and $\mathrm{Zn}$ also accumulate from the water column under $\mathrm{SO}_{4}{ }^{2-}$-reducing redox conditions. Their rates of deposition, and that of Mo, are controlled by the level of their removal from the bottom water, relative to their concentrations in the source water, and the residence time of bottom water. The initial composition of the bottom water for the entire period of deposition of the sediment core is taken as equal to the current composition of water between 100 and 250-m depth in the basin (tables 2 and 6). Water at this approximate depth was shown by Jacobs and others (1987) to be the source of water currently fluxing into the deeper region of the basin. It is also considered the source of water upwelling into the photic zone (Müller-Karger and others, 2001). As noted above, the residence time of bottom water is about $100 \mathrm{yr}$ (Deuser, 1973).

Based on analyses of the water column of this and additional modern basins, the degree of removal of $\mathrm{Cd}$ and $\mathrm{Cu}$ from the source water, within the deep part of the basin, is essentially independent of the concentration of $\mathrm{H}_{2} \mathrm{~S}$ (fig. 10A). By contrast, the removal of Mo in the bottom water from the source water is strongly dependent upon the concentration of $\mathrm{H}_{2} \mathrm{~S}$ (fig. 10B). The removal of $\mathrm{V}$ from the source water versus $\mathrm{H}_{2} \mathrm{~S}$ is inexplicably complex (fig. 10B). In the Cariaco Basin, $\Delta \mathrm{V}$ is 65 percent (fig. 10B). Ni profiles of other basins suggest that $\mathrm{Ni}$ is not removed from the bottom water under $\mathrm{SO}_{4}{ }^{2-}$-reducing conditions. $\mathrm{Zn}$ has not been measured in the Cariaco Basin, to our knowledge, but its profile in the other basins that exhibit $\mathrm{SO}_{4}{ }^{2-}$ reduction in the bottom water parallels the profiles of $\mathrm{Cd}$ and $\mathrm{Cu}$ (fig. 10A). The same is considered to apply to the Cariaco Basin. Its concentration in the source water is assumed to equal its concentration in the open ocean of the North Atlantic (table 6). Emerson and others (1979) and Murray and others (1983) have shown that $\mathrm{Cr}$ is reduced to the insoluble $3+$ valence state under denitrifying to $\mathrm{SO}_{4}{ }^{2-}$-reducing conditions, but its distribution has been reported only for Saanich Inlet and the $\mathrm{O}_{2}$ minimum zone of the eastern Pacific Ocean. Still, the depositional rates of Cd, $\mathrm{Cu}, \mathrm{Ni}, \mathrm{V}$, and $\mathrm{Zn}$ offer a check on our interpretation of the deposition and eventual accumulation of Mo as defining the level of $\mathrm{SO}_{4}{ }^{2-}$ reduction in the water column and bottom-water advection.

In summary, the depositional rate of each trace element in the surface sediment is calculated from the sum of its rain rate onto the sea floor within the three source fractions. The calculations are based on (1) the current depositional rate of terrigenous debris and its composition, (2) the flux of organic matter into the bottom water of the basin and its trace-element
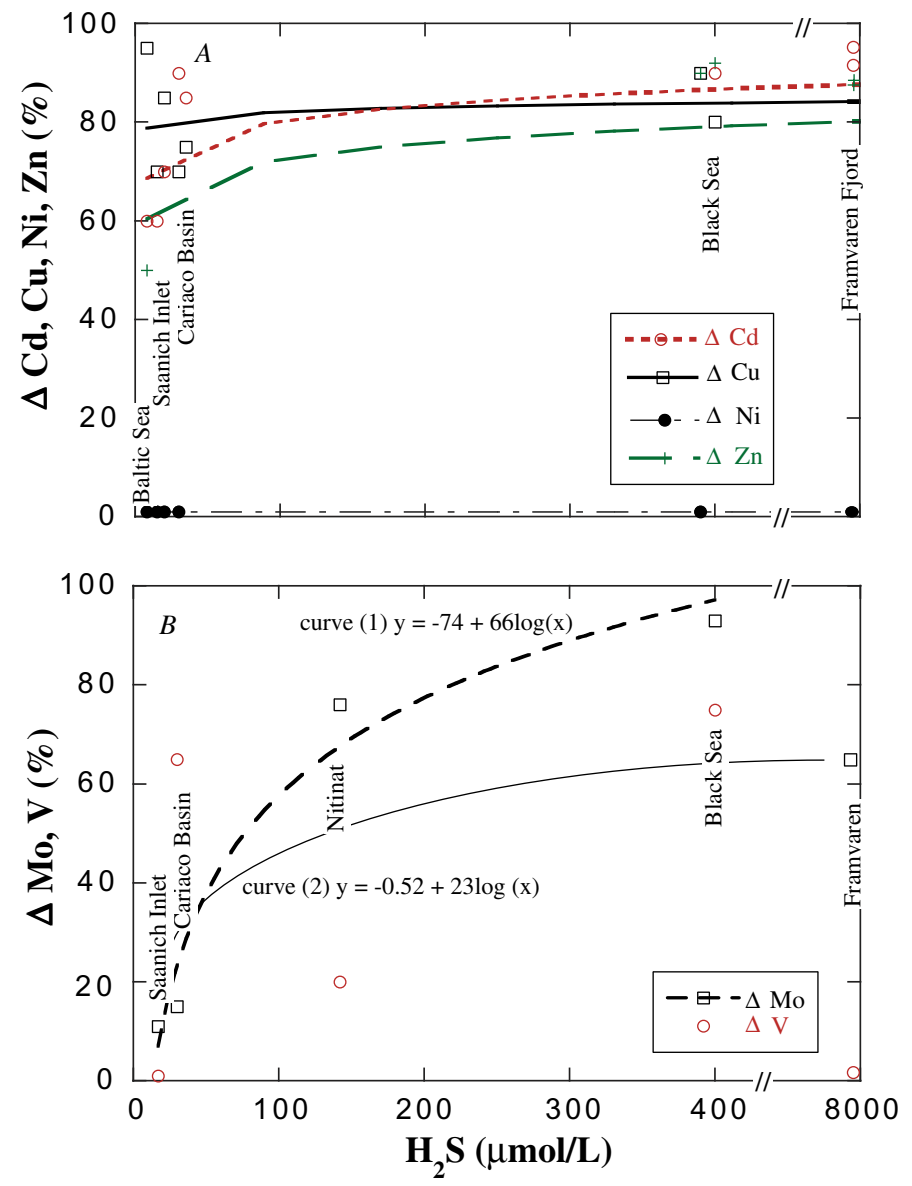

Figure 10. The percentage of $\mathrm{Cd}, \mathrm{Cu}, \mathrm{Ni}$, and $\mathrm{Zn}(A)$ and $\mathrm{Mo}$ and $\mathrm{V}(B)$ removed from $\mathrm{SO}_{4}{ }^{2-}$-reducing bottom waters of modern marine basins (Brewer and Spencer, 1974; Kremling, 1983; Jacobs and others, 1985, 1987; Emerson and Huested, 1991; Landing and Lewis, 1991), relative to their mean concentrations in the sources of bottom water, versus the mean concentration of $\mathrm{H}_{2} \mathrm{~S}$ in the bottom waters. The curves represent regressions of best fit, of the form $y=C 1+C 2 \log [x]$, for which $R=0.75(\mathrm{Cd}), 0.31(\mathrm{Cu}), 0.00$ (Ni), 0.70 $(\mathrm{Zn})$. The equation for curve (1) for $\Delta$ Mo versus $\mathrm{H}_{2} \mathrm{~S}(B), \mathrm{R}=0.96$, is used in the calculations in table 7. It does not include the datum from Framvaren Fjord. Curve (2), $R=0.66$, includes all Mo data. Depth profiles of Cd, Cu, and Zn in the Baltic Sea, Black Sea, and Framvaren Fjord exhibit strong depletions in the bottom water; from which all three may precipitate as sulfides (Jacobs and others, 1985). In the Cariaco Basin, only Cd and Cu have been measured, where they also are strongly depleted in the bottom water (Jacobs and others, 1987). Zn has not been reported, but its depletion in the other basins suggests that its depletion in the bottom water of the Cariaco Basin is similar to that of $\mathrm{Cd}$ and $\mathrm{Cu}$. The concentration of $\mathrm{Ni}$ is essentially constant with depth in all of the basins for which it has been reported. The relation between $\Delta \mathrm{V}$ and $\mathrm{H}_{2} \mathrm{~S}$ clearly does not exhibit a simple regression.

stoichiometry, and (3) the residence time of bottom water and its depletion of trace elements relative to the trace-element concentrations of the water advecting into the deep region of the basin. The model defines the relationship of the deposition of trace elements to upwelling of seawater into the photic zone and advection of seawater into the deeper region of the basin. 


\section{Trace-Element Depositional Rates in the Surface Sediment-A Test of the Model}

Marine trace-element sources are (1) organic matter that escapes oxidation in the oxic layers of the water column and settles into the bottom water and onto the sea floor and (2) the dissolved load of trace elements in bottom water that are removed by precipitation/adsorption reactions in the water column (Goldberg, 1963; Brewer and Spencer, 1974; Jacobs and Emerson, 1982; Jacobs and others, 1985, 1987; Helz and others, 1996) and at the benthic boundary (Francois, 1988; Emerson and Huested, 1991; Crusius and others, 1996). The biogenic contribution of $\mathrm{Cd}$ is calculated from the export of organic matter from the photic zone into the $\mathrm{SO}_{4}{ }^{2-}$-reducing region of the water column and its calculated concentration in plankton for the Venezuela Basin of $5.5 \mathrm{ppm}$ (table 2). The flux of organic carbon measured by Thunell and others (2000) and Müller-Karger and others (2001) is converted to the flux of organic matter (table 6), based on the stoichiometry of plankton (Redfield and others, 1963). The mean rain rate of organic matter at $255 \mathrm{~m}$ is $8.89 \mathrm{mg} \mathrm{cm}^{-2} \mathrm{yr}^{-1}$ (Müller-Karger and others, 2000), giving a depositional rate for $\mathrm{Cd}$ in the organic fraction of $0.049 \mu \mathrm{g} \mathrm{cm}^{-2} \mathrm{yr}^{-1}$. Compared to the measured bulk accumulation rate for the surface sediment in the core of $0.061 \mu \mathrm{g} \mathrm{cm}^{-2} \mathrm{yr}^{-1}$, the marine biogenic fraction alone contributes 80 percent to the bulk sediment value (fig. 11).

A lower $\mathrm{Cd}$ concentration in the $\mathrm{SO}_{4}{ }^{2-}$-reducing part of the water column (Jacobs and others, 1987) than in the overlying, denitrifying part of the water column reflects $\mathrm{Cd}$ precipitation from the bottom water by a direct, albeit complex, reaction with $S^{2-}$ (Jacobs and others, 1985). Its deposition (fig. 11 ; table 6) is calculated from (1) the difference between its concentration in the $\mathrm{SO}_{4}{ }^{2-}$-reducing part of the water column and the above region of the water column, (2) the thickness of the $\mathrm{SO}_{4}{ }^{2-}$-reducing portion of the water column above the core site of about $600 \mathrm{~m}$, or $60 \mathrm{~L} \mathrm{~cm}^{-2}$, and (3) the residence time of bottom water of $100 \mathrm{yr}$ (Deuser, 1973). Cd removal from the bottom water gives a depositional rate of $0.006 \mu \mathrm{g} \mathrm{cm}^{-2} \mathrm{yr}^{-1}$.

The depositional rate of the terrigenous fraction currently is $7.36 \mathrm{mg} \mathrm{cm}^{-2} \mathrm{yr}^{-1}$, requiring approximately 30 percent of the total of the sediment-trap material collected at $950 \mathrm{~m}$, of $25 \mathrm{mg}$ $\mathrm{cm}^{-2} \mathrm{yr}^{-1}$ (Thunell and others, 2000), to be terrigenous in origin. Although the 30 percent seems to be a reasonable value, $\mathrm{Al}_{2} \mathrm{O}_{3}$, biogenic $\mathrm{SiO}_{2}$, and $\mathrm{CaCO}_{3}$, unfortunately, were not reported. The $\mathrm{Cd}$ accumulating as a terrigenous fraction, $0.002 \mu \mathrm{g} \mathrm{cm}^{-2}$ $\mathrm{yr}^{-1}$, reflects its concentration in and deposition of the terrigenous debris (table 2, fig. 11). The $\mathrm{Cd}$ accumulation-rate sum for the three fractions is $0.057 \mu \mathrm{g} \mathrm{cm}^{-2} \mathrm{yr}^{-1}$, compared to a mea-

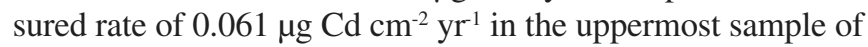
sediment. The agreement of the two values is extremely good, considering the possible uncertainties discussed previously. Of course, uncertainties will become greater as the calculations are applied to sediment at depth in the core. The agreement also suggests that the sediment largely settled through the water column rather than having been derived from the sides of the basin by resuspension of previously deposited material.
Even so, the texture of some individual laminae at depth in the core clearly reflects mass movement of sediment down slope (Hughen and others, 1996a).

The measured $\mathrm{Zn}$ accumulation rate in the surface sediment is $1.187 \mu \mathrm{g} \mathrm{cm}^{-2} \mathrm{yr}^{-1}$; the calculated depositional-rate sum is $1.763 \mu \mathrm{g} \mathrm{cm}^{-2} \mathrm{yr}^{-1}$ (table 6). Its rate calculated within the biogenic fraction may account for most of this discrepancy. Whereas the concentration of $\mathrm{Zn}$ in plankton estimated from actual measurements is $110 \mathrm{ppm}$, its seawater profile with $\mathrm{PO}_{4}^{3-}$ in the North Atlantic Ocean gives a value of $30 \mathrm{ppm}$ (Bruland and Franks, 1983). Using this stoichiometric value reduces the calculated biogenic contribution and improves agreement between the calculated and measured rates. The measured accumulation rate of $\mathrm{Cu}$ is $0.318 \mu \mathrm{g} \mathrm{cm}^{-2} \mathrm{yr}^{-1}$ and the calculated sum is $0.339 \mu \mathrm{g} \mathrm{cm}^{-2} \mathrm{yr}^{-1}$. Ni shows a level of agreement between the calculated and measured rates that is only slightly poorer (table 6). Thus, the biogenic contribution of $\mathrm{Cd}, \mathrm{Cu}, \mathrm{Ni}$, and $\mathrm{Zn}$ dominates their marine fraction; it dominates the bulk accumulation rate only of $\mathrm{Cd}$.

A minimum rate of upwelling can be estimated from the export of these four trace elements from the oxic region of the water column, that is, export of organic matter, or new production (Eppley and Peterson, 1979), and their concentrations in upwelled water. For $\mathrm{Cd}$, the upwelling rate equals $\left(0.049 \mu \mathrm{g} \mathrm{cm}^{-2} \mathrm{yr}^{-1} \div 0.011 \mu \mathrm{g} \mathrm{L}^{-1}\right) \times 1,000 \mathrm{~cm}^{3} \mathrm{~L}^{-1}=45 \mathrm{~m} \mathrm{yr}^{-1}$. The upwelling rate is higher by the loss of Cd laterally across the shelf into the Venezuela Basin, in the Ekman layer. The maximum upwelling rate will be the total uptake rate of $\mathrm{Cd}$ in the photic zone divided by its concentration in the upwelling water, or approximately $760 \mathrm{~m} \mathrm{yr}^{-1}$.

The accumulation rate of Mo is dominated by the hydrogenous fraction. Its water-column depth profile (Jacobs and others, 1987; Emerson and Huested, 1991) reflects a mean removal in the $\mathrm{SO}_{4}^{2-}$-reducing part of the water column of 15 percent of its concentration in the uppermost $250 \mathrm{~m}$ of the water column. Its depositional rate as a hydrogenous fraction is $0.963 \mu \mathrm{g} \mathrm{cm}^{-2} \mathrm{yr}^{-1}$ (table 6, fig. 11), as a terrigenous fraction $0.015 \mu \mathrm{g} \mathrm{cm}^{-2} \mathrm{yr}^{-1}$, and as a biogenic fraction $0.018 \mu \mathrm{g} \mathrm{cm}^{-2} \mathrm{yr}^{-1}$. The latter two represent only 3 percent of the bulk Mo accumulation rate. Again, the agreement between the measured and calculated accumulation rates is excellent.

The closeness of the two Mo accumulation rates is somewhat surprising, considering decadal changes in the concentration of $\mathrm{H}_{2} \mathrm{~S}$ within the water column of as much as 30 percent (Richards, 1975; Scranton and others, 1987) and the relation between the removal of Mo from the water column and the concentration of $\mathrm{H}_{2} \mathrm{~S}$ (fig. $10 \mathrm{~B}$ ). As an individual sample represents a minimum of approximately 25 years under our sediment-sampling scheme, each sample averages over time scales of several decades. A finer sampling scheme of cores of undisturbed sediment is required to evaluate decadal and shorter trends in bottom-water chemistry.

The mean rate of advection of water into the bottom of the basin is much less intense than the upwelling rate. It is simply the total thickness of the $\mathrm{O}_{2}$-depleted region of the water column divided by the residence time of bottom water, or approximately $1,200 \mathrm{~m} \div 100 \mathrm{yr}=12 \mathrm{~m} \mathrm{yr}^{-1}$. 


\section{Marine Input}

Hydrogenous Source seawater concentration (ppb)

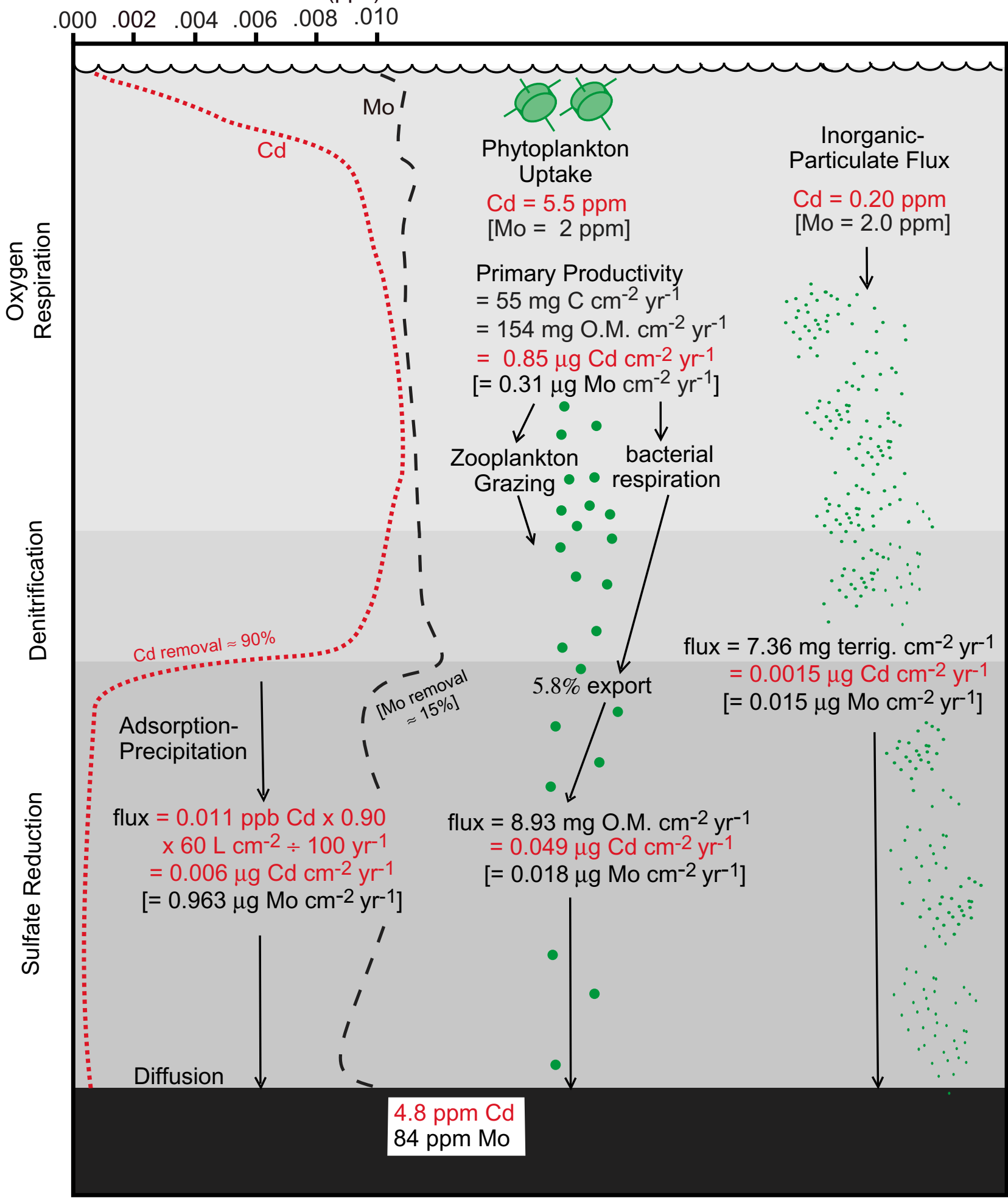

Figure 11. Schematic for the Cariaco Basin, showing the relation of "model" accumulation rates of the marine and terrigenous fractions of $\mathrm{Cd}$ and $\mathrm{Mo}$ (in parenthesis) to current conditions of primary productivity, water-column redox, bottom-water residence time, and terrigenous input. Depth ranges are not to scale. The two curves in the left side of the figure represent approximate seawater concentration versus depth profiles for $\mathrm{Mo}\left(\times 10^{-3}\right)$ and $\mathrm{Cd}$. Model concentrations of $\mathrm{Cd}$ and Mo in the sediment, given at the bottom of the figure, compare closely with their measured concentrations in the surface sediment (table 1). 
Removal of trace elements from the bottom water (precipitation or adsorption onto settling particles or diffusion across the benthic boundary) is likely different for the different trace elements (Jacobs and others, 1985). Adsorption and diffusion reactions at the benthic boundary layer have been advocated as the mechanisms for the deposition of Mo in this and similar sedimentary environments (Francois, 1988; Pedersen and others, 1989; Emerson and Huested, 1991; Crusius and others, 1996), whereas $\mathrm{Cd}, \mathrm{Cu}$, and $\mathrm{Zn}$ are deposited as sulfides (Jacobs and others, 1985). Whichever mechanism(s) might account for $\mathrm{Cd}, \mathrm{Cu}, \mathrm{Mo}, \mathrm{Ni}, \mathrm{V}$, and $\mathrm{Zn}$ removals from bottom water, it appears that their rates of deposition reflect the chemistry and hydrography of bottom water rather than the chemistry of sediment pore water. The agreement between model depositional rates of the different trace elements with their measured accumulation rates further supports the values used for their concentrations in planktonic debris, siliciclastic debris, and seawater. Baring temporal changes in these values, which are not possible to ascertain, and any diagenetic redistribution of the elements, it should be possible to ascertain historical changes in primary productivity and bottom-water residence times within approximately 25 percent.

\section{Trace-Element Accumulation Rates During The Last 20,000 Years-An Application of the Model}

The excellent agreement between the calculated and measured accumulation rates of $\mathrm{Cd}, \mathrm{Cu}, \mathrm{Mo}, \mathrm{Ni}$, and $\mathrm{Zn}$ in the surface sediment allows changes in the measured trace-element accumulation rates at depth in the sediment core to be interpreted in terms of past changes in (1) provenance, (2) primary productivity, and (3) bottom-water residence time and $\mathrm{H}_{2} \mathrm{~S}$ concentration. Bottom water contributes less to the accumulations of $\mathrm{Cd}, \mathrm{Cu}, \mathrm{Ni}$, and $\mathrm{Zn}$ than the rain rate of organic matter and should do so throughout the full range of biological and geochemical conditions. Thus, the distributions of marine $\mathrm{Cd}, \mathrm{Cu}$, $\mathrm{Ni}$, and Zn may have recorded historical levels of primary productivity, that is, of the export of organic matter from the photic zone to the bottom water and seafloor. We focus on Cd because of its minor contribution by bottom water (table 6) and its low concentration in the terrigenous siliciclastic debris (table 2).

In relating the marine $\mathrm{Cd}$ and other trace-element accumulation rates to the hydrography of the basin, several assumptions must be made about the geochemistry of the source fractions: (1) phytoplankton maintained a constant stoichiometry of $5.5 \mathrm{ppm} \mathrm{Cd}$, (2) Cd removal from bottom water under conditions of $\mathrm{SO}_{4}{ }^{2-}$ reduction was constant at 90 percent over the full range of $\mathrm{H}_{2} \mathrm{~S}$ concentrations considered (fig. 10A), (3) water fluxing into the bottom water had a constant $\mathrm{Cd}$ concentration of $0.011 \mathrm{ppb}$, the concentration of $\mathrm{Cd}$ in the water column currently at 100 to $250 \mathrm{~m}$ (Jacobs and others, 1987), (4) the Cd concentration in terrigenous debris fluxing into the basin was $0.2 \mathrm{ppm}$ and constant, and (5) organic matter exported from the photic zone increased as a percentage of primary productivity. Any historical variations in the trace-element composition of plankton and seawater that may have occurred over the past 20,000 yr represent possibly sources of error that can be merely acknowledged.

The fifth assumption requires some explanation. The export of organic matter from the photic zone possibly does not bear a simple linear relationship with primary productivity. Baines and others (1994) showed that, of the organic matter produced in the photic zone, the percentage exported from the photic zone increases as primary productivity increases. Müller-Karger and others (2001) concluded that, for the Cariaco Basin, the percentage of organic matter settling out of the photic zone was constant throughout the range of primary productivity. They calculated regression curves from their measurements of primary productivity and the flux of organic-matter at four depths in the water column (their fig. 11). The curve representing the sediment-trap data at a water depth of $275 \mathrm{~m}$, alone, predicted a flux that remained a constant percentage of primary productivity throughout the full range of primary productivity. Sediment traps at three other depths recorded a percentage increase in export with increasing primary productivity. At a primary productivity of $500 \mathrm{mg} \mathrm{C} \mathrm{m}^{-2} \mathrm{~d}^{-1}$, traps at $455 \mathrm{~m}, 930 \mathrm{~m}$, and $1,225 \mathrm{~m}$ collected organic matter equaling one percent of primary productivity. At a primary productivity of $2,500 \mathrm{mg} \mathrm{C} \mathrm{m}^{-2} \mathrm{~d}^{-1}$, the percentage increased to 4.6 percent at $433 \mathrm{~m}, 2.6$ percent at $930 \mathrm{~m}$, and 1.7 percent at $1,225 \mathrm{~m}$. An increase in the percentage flux at $275 \mathrm{~m}$ as well as at the other three depths, from approximately 1 percent to 6.8 percent over the same range of primary productivity, is obtained if the datum at a primary productivity of $475 \mathrm{mg} \mathrm{C} \mathrm{m}^{-2} \mathrm{~d}^{-1}$ is deleted. Deletion of this single measurement is similar to the authors' deletion of two measurements that they interpreted as being outliers. Thus, we interpret their figure 11 as showing that all four sediment traps recorded percentage increases in the flux of organic matter with increasing primary productivity. This interpretation of a percentage increase in the export of organic matter out of the photic zone under the condition of increasing primary productivity is supported by the clogging of sediment traps at all depths, immediately following their deployment in May of 1996. Müller-Karger and others (2001) attributed the event to a plankton bloom observed in that month. They did not consider the event in their model. Such events, how ever brief and few in number, might provide for an even greater percentage of primary productivity to be exported out of the photic zone than our calculations suggest (table 7). Even if that is not fully the case, the large flux of organic matter settling into the bottom water from this single event suggests that the flux of organic matter settling into the bottom water versus primary productivity is far more complex than a simple linear relation between the two.

This interpretation is used to model the flux of organic matter and trace nutrients $(\mathrm{Cd})$ out of the photic zone and into the bottom water (table 7). Doubling primary productivity, for example, from $1.5 \mathrm{~g} \mathrm{C} \mathrm{m}^{-2} \mathrm{~d}^{-1}\left(550 \mathrm{~g} \mathrm{C} \mathrm{m}^{-2} \mathrm{yr}^{-1}\right)$ to $3 \mathrm{~g} \mathrm{C} \mathrm{m}^{-2} \mathrm{~d}^{-1}\left(1100 \mathrm{~g}^{-}\right.$ $\mathrm{C} \mathrm{m}^{-2} \mathrm{yr}^{-1}$ ), increases the export of organic carbon from the photic zone from 5.8 percent to 6.7 percent of primary productivity, or 
Table 7. Table 7. Calculated rates of deposition for the Cariaco Basin of marine Mo and Cd (1) as particulate organic matter settling out of the photic zone (the biogenic fraction) and (2) from bottom water under conditions of $\mathrm{SO}_{4}^{2-}$ reduction (the hydrogenous fraction).

[Calculations are made for four different rates of primary productivity in the photic zone and several different residence times of bottom water for each level of primary productivity. $\mathrm{H}_{2} \mathrm{~S}$ concentrations are based on its current concentration in the Cariaco Basin of approximately $30 \mu \mathrm{mol} \mathrm{L}-1$ (Emerson and Huested, 1991), at the residence time of bottom water of $100 \mathrm{yr}$ and primary productivity of $550 \mathrm{~g}$ carbon $\mathrm{m}^{-2} \mathrm{yr}^{-1}$. $\mathrm{H}_{2} \mathrm{~S}$ concentration is allowed to change 1-to-1 with a change in residence time. It is allowed to change with primary productivity by the percentage change in export of organic matter from the photic zone to $255-\mathrm{m}$ depth (see text). $\Delta$ Mo values are taken from the curve given in figure $10 B$. Mo depositional rate equals $\left[\Delta \mathrm{Mo} \times 60 \mathrm{~L} \mathrm{~cm}-2 \times 10.7 \mu \mathrm{g} \mathrm{Mo} \mathrm{L-1]} \div\left[\sigma_{\tau}\right.\right.$ yr], for which $\sigma_{\tau}$ is the bottom-water residence time. $\Delta \mathrm{Cd}$ (not listed) is held constant at 90 percent, its current removal from the Cariaco Basin and roughly that for the full range of $\mathrm{H}_{2} \mathrm{~S}$ concentrations as observed in other basins (fig. 10A)]

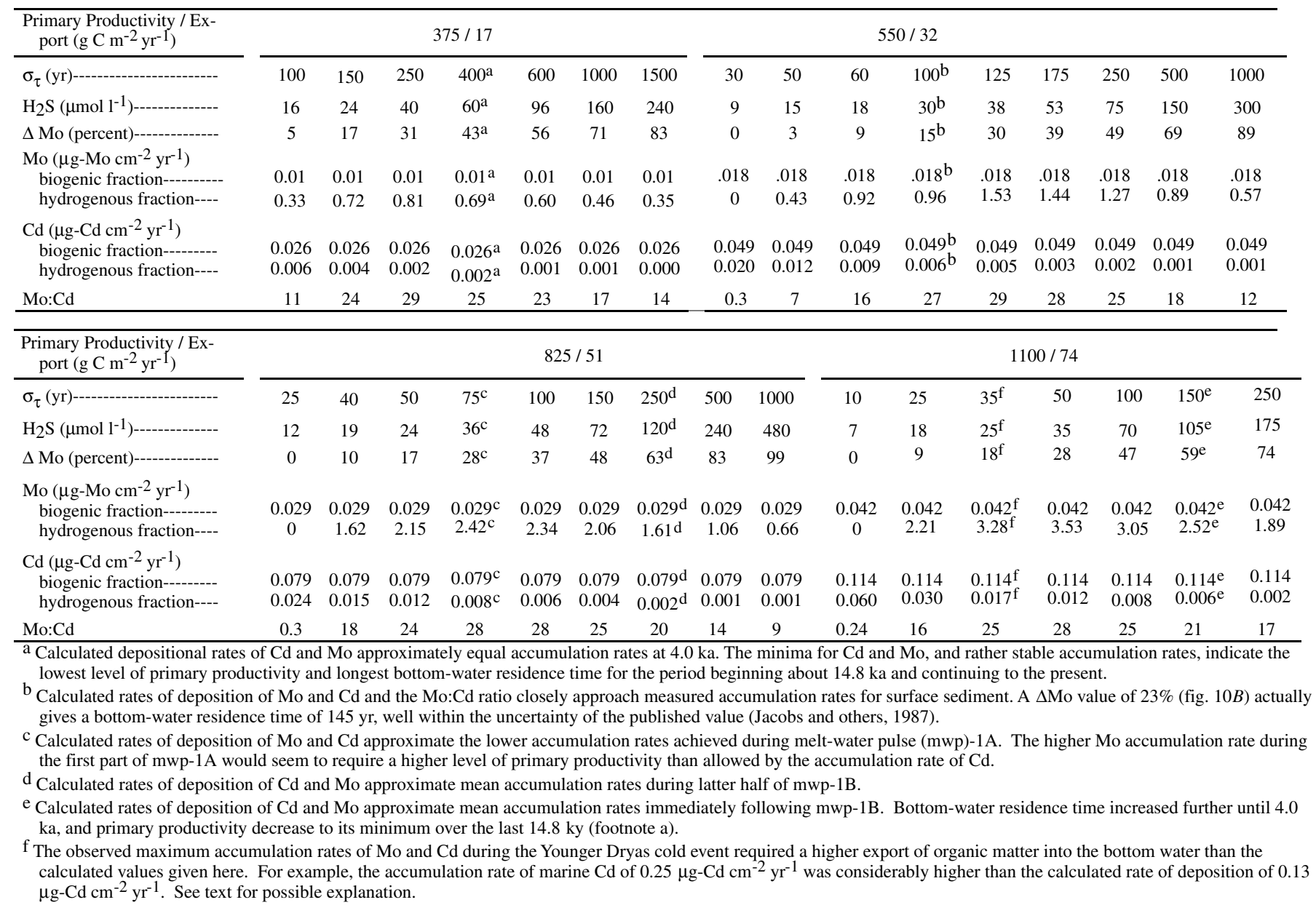

from $32 \mathrm{~g} \mathrm{C} \mathrm{m}^{-2} \mathrm{yr}^{-1}$ to $74 \mathrm{~g} \mathrm{C} \mathrm{m}^{-2} \mathrm{yr}^{-1}$ (table 7). Export of the nutrient trace elements will increase proportionally.

The model calculations of $\mathrm{H}_{2} \mathrm{~S}$ in the bottom water begin by taking the current conditions of primary productivity (550 $\mathrm{g} \mathrm{C} \mathrm{m}^{-2} \mathrm{~d}^{-1}$ ), bottom-water residence time (100 yr) and $\mathrm{H}_{2} \mathrm{~S}$ concentration in the bottom water $\left(30 \mu \mathrm{mol} \mathrm{L}^{-1}\right)$. The concentration of $\mathrm{H}_{2} \mathrm{~S}$ in the bottom water is then allowed to change by (1) the rate of bacterial respiration, which is established by the flux of labile organic matter into the bottom water and, ultimately, by primary productivity, and (2) bottom-water advection, or residence time (Codispoti, 1980). As the production of $\mathrm{H}_{2} \mathrm{~S}$ is inextricably coupled both to primary productivity and bottom-water advection, its concentration cannot be considered solely as a function of one or the other. The removal of trace elements from bottom water, likewise, will be dependent upon both factors. Conceptually, the response of $\mathrm{H}_{2} \mathrm{~S}$ in the bottom water to a change in each can be considered separately.
The concentration of $\mathrm{H}_{2} \mathrm{~S}$ should change one-to-one with a change in bottom-water residence time. Doubling the residence time of bottom water simply doubles the integrated flux of organic matter settling through a parcel of water and the concentration of $\mathrm{H}_{2} \mathrm{~S}$ (table 7), assuming that the rate of bacterial respiration is independent of the concentration of $\mathrm{H}_{2} \mathrm{~S}$. At the low concentrations of $\mathrm{H}_{2} \mathrm{~S}$ in the Cariaco Basin of $30 \mu \mathrm{mol}$ $\mathrm{L}^{-1}$ (Emerson and Huested, 1991), this is a reasonable assumption. Holding residence time constant, a change in the concentration of $\mathrm{H}_{2} \mathrm{~S}$ will equal the change in the flux of organic matter into the bottom water, as driven by a change in primary productivity. Doubling primary productivity will increase somewhat more than twofold the flux of settling organic matter, bacterial respiration, and the concentration of $\mathrm{H}_{2} \mathrm{~S}$.

The percent removal of $\mathrm{Cd}, \mathrm{Cu}$, and $\mathrm{Zn}$, from bottom water, their hydrogenous fraction, responds weakly to a change in the concentration of $\mathrm{H}_{2} \mathrm{~S}$ (fig. 10A). Their removal 
is about 70 to 90 percent throughout the full range of $\mathrm{H}_{2} \mathrm{~S}$ concentrations, from the low concentration of $\mathrm{H}_{2} \mathrm{~S}$ in the Baltic Sea (Kremling, 1983) to the highest concentration of $\mathrm{H}_{2} \mathrm{~S}$ measured for the bottom water of Framvaren Fjord (Jacobs and others, 1985). Ni is not removed from the bottom water by reaction with $\mathrm{S}^{2-}$ (Jacobs and others, 1985), above the contribution of $\mathrm{Ni}$ added directly to the water column via the oxidation of settling organic matter, even at the high levels of $\mathrm{H}_{2} \mathrm{~S}$ in the water column of Framvaren Fjord. Thus, a change in the concentration of $\mathrm{H}_{2} \mathrm{~S}$ in bottom water, alone, does not change significantly the accumulation rates of any of these four trace elements.

The Mo removal from bottom water is complicated by the relation between $\Delta \mathrm{Mo}$ and the concentration of $\mathrm{H}_{2} \mathrm{~S}$ (fig. $10 B)$. The percentage depletion of Mo in the bottom water of modern basins exhibiting $\mathrm{O}_{2}$ depletion versus $\mathrm{H}_{2} \mathrm{~S}$ is nonlinear over the full range of $\mathrm{H}_{2} \mathrm{~S}$ concentrations. As a result, the Mo accumulation rate can exceed an increase in $\mathrm{H}_{2} \mathrm{~S}$, in response to an increase in primary productivity. For example, a threefold increase in $\mathrm{H}_{2} \mathrm{~S}$, from $15 \mu \mathrm{mol} \mathrm{L}^{-1}$ to $45 \mu \mathrm{mol} \mathrm{L}{ }^{-1}$, increases $\Delta \mathrm{Mo}$ from 3.6 to 35 percent (fig. 10B). Therefore, a threefold increase in primary productivity can increase the Mo accumulation rate as much as tenfold, at low $\mathrm{H}_{2} \mathrm{~S}$ concentrations. At $\mathrm{H}_{2} \mathrm{~S}$ concentrations above about $150 \mu \mathrm{mol} \mathrm{L}^{-1}, \Delta \mathrm{Mo}$ increases only slightly with an increase in $\mathrm{H}_{2} \mathrm{~S}$ (fig. $10 \mathrm{~B}$, table 7). The increase in removal of Mo from bottom water under higher $\mathrm{H}_{2} \mathrm{~S}$ concentrations (fig. $10 B$ ) is compensated increasingly at long residence times by the reduced flux of bottom water, that is, of dissolved Mo and other trace elements, into the basin. Under a rate of primary productivity of $1,100 \mathrm{~g} \mathrm{C} \mathrm{m}^{-2}$ $\mathrm{yr}^{-1}$, an increase in residence time from $50 \mathrm{yr}$ to $250 \mathrm{yr}$ (table 7) increases by fivefold the concentration of $\mathrm{H}_{2} \mathrm{~S}$ in bottom water and the removal of Mo from the water column by about 2.5fold (fig. 10B). However, the accumulation rate of Mo actually decreases by twofold at the longer bottom-water residence time, owing to the fivefold reduction in the flux of Mo into the bottom water.

Increasing the bottom-water residence time reduces the accumulation rates of $\mathrm{Cd}, \mathrm{Cu}$, and $\mathrm{Zn}$ from bottom water, approximately one-to-one throughout the range of $\mathrm{H}_{2} \mathrm{~S}$ concentrations (table 7). That is, doubling the residence time of bottom water halves their rate of accumulation within the hydrogenous fraction by halving their flux into the basin. Changing the $\mathrm{H}_{2} \mathrm{~S}$ concentration by changing the residence time should not alter the accumulation rate of $\mathrm{Ni}$.

Modeling the Mo accumulation from the hydrography of a basin that exhibits $\mathrm{SO}_{4}{ }_{4}^{2-}$ reduction in the bottom water (table 7) shows several features that are critical to our interpretation of the sediment record: (1) Large temporal variations in the Mo accumulation rate are more easily achieved for brief bottom-water residence times than for bottom-water residence times of long duration. For example, at a primary productivity of $825 \mathrm{~g} \mathrm{C} \mathrm{m}^{-2} \mathrm{yr}^{-1}$, a decrease in residence time, from 1000 $\mathrm{yr}$ to $500 \mathrm{yr}$, increases the accumulation rate of Mo $0.4 \mu \mathrm{g}$ $\mathrm{cm}^{-2} \mathrm{yr}^{-1}$ (table 7). Increasing the residence time from $40 \mathrm{yr}$ to $50 \mathrm{yr}$, similarly increases the Mo accumulation rate about $0.4 \mu \mathrm{g} \mathrm{cm}^{-2} \mathrm{yr}^{-1}$. Therefore, a Mo accumulation-rate pattern that exhibits sharp temporal changes over a period of several thousand years is indicative of a bottom-water residence time that varied over a few tens of years, whereas a pattern that shows a gradual change in the Mo accumulation rate suggests a bottom-water residence time that varied between one hundred and several hundred years. (2) The accumulation rate of $\mathrm{Cd}$ is practically independent of bottom water residence time, owing to its low accumulation rate within the hydrogenous fraction. (3) A Mo:Cd ratio less than one can be achieved only at bottom-water residence times less than a few years or greater than several thousand years. The former was probably never sustained for a time long enough to have been detected by our sampling scheme; the latter is simply unlikely to have occurred. (4) Mo exhibits a maximum accumulation rate at a decreasing bottom-water residence time as primary productivity increases.

Several of the temporal changes in trace-element accumulation rates in the Cariaco Basin (fig. 12) are approximately synchronous with global climatic events, although accumulation of the trace elements within any single event exhibits strong variability. Thus, our results must be taken as preliminary until a more detailed examination of the geochemistry of this and similar sedimentary basins more firmly constrains the relations between trace-element accumulation and chemical, biological, and hydrographic properties of the water column.

In the following discussion, the accumulation rates of the trace elements from $20 \mathrm{ka}$ to the present are considered. Prior to about $17 \mathrm{ka}$, the entire water column was oxic (Peterson and others, 1991). Additionally, much of the shelf was exposed during this earlier period. The source of the terrigenous fraction of sediment could have been quite different from its current source. Whereas the Orinoco/Amazon River systems now contribute substantially to the terrigenous fraction, they may not have done so prior to about $15 \mathrm{ka}$ (Clayton and others, 1999). Owing to this and other factors, the geochemistry of the sediment may not have retained an accurate record of the dynamics and chemistry of the immediately overlying water column, nor recorded conditions that can be related to the current oceanographic regime. Following this time, the sediment record of the Cariaco Basin can provide a relatively high-resolution record of the hydrography of water-column because of the high bulk-sediment accumulation rate, between 10 and 85 $\mathrm{mg} \mathrm{cm}^{-2} \mathrm{yr}^{-1}$. Of course, the adverse effect of a higher accumulation rate is increased dilution of the marine trace-element record by terrigenous debris, $\mathrm{CaCO}_{3}$, and biogenic $\mathrm{SiO}_{2}$.

Calculations of the accumulation rates for $\mathrm{Cr}$ and $\mathrm{V}$ (fig. 12) are included, but are not discussed in any depth. The concentration of $\mathrm{Cr}$ in the water column is not known to have been measured. This is rather unfortunate as it and $\mathrm{V}$ as well are removed from the water column by precipitation and adsorption reactions under conditions of denitrification and continue to be deposited under conditions of $\mathrm{SO}_{4}{ }^{2-}$ reduction (Piper, 1994). It should be noted that the mean concentration of $\mathrm{Cr}$ in the laminated sections of the core, relative to Mo (table 2), is similar to their relative concentrations in open-ocean seawater, Mo greater than $\mathrm{Cr}$, but the $\mathrm{Cr}$ concentration in the water column must be measured before considering this element further. 
Calendar Age (ka)

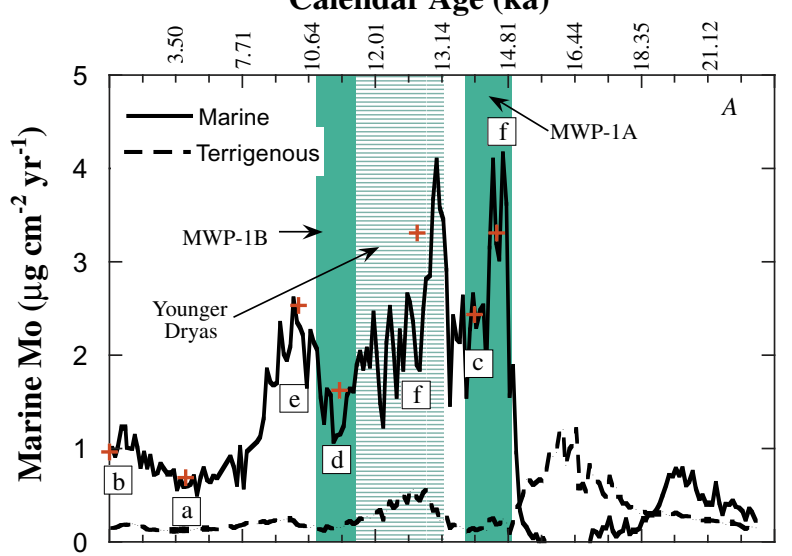

0.5

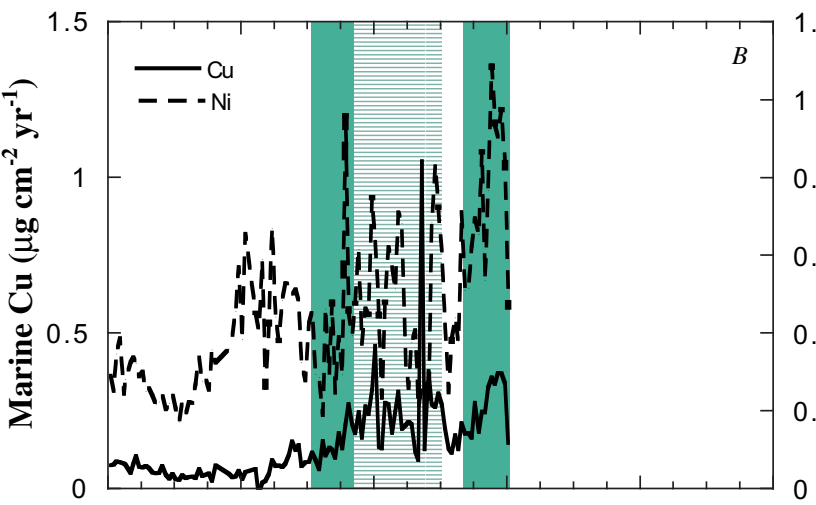

1.2
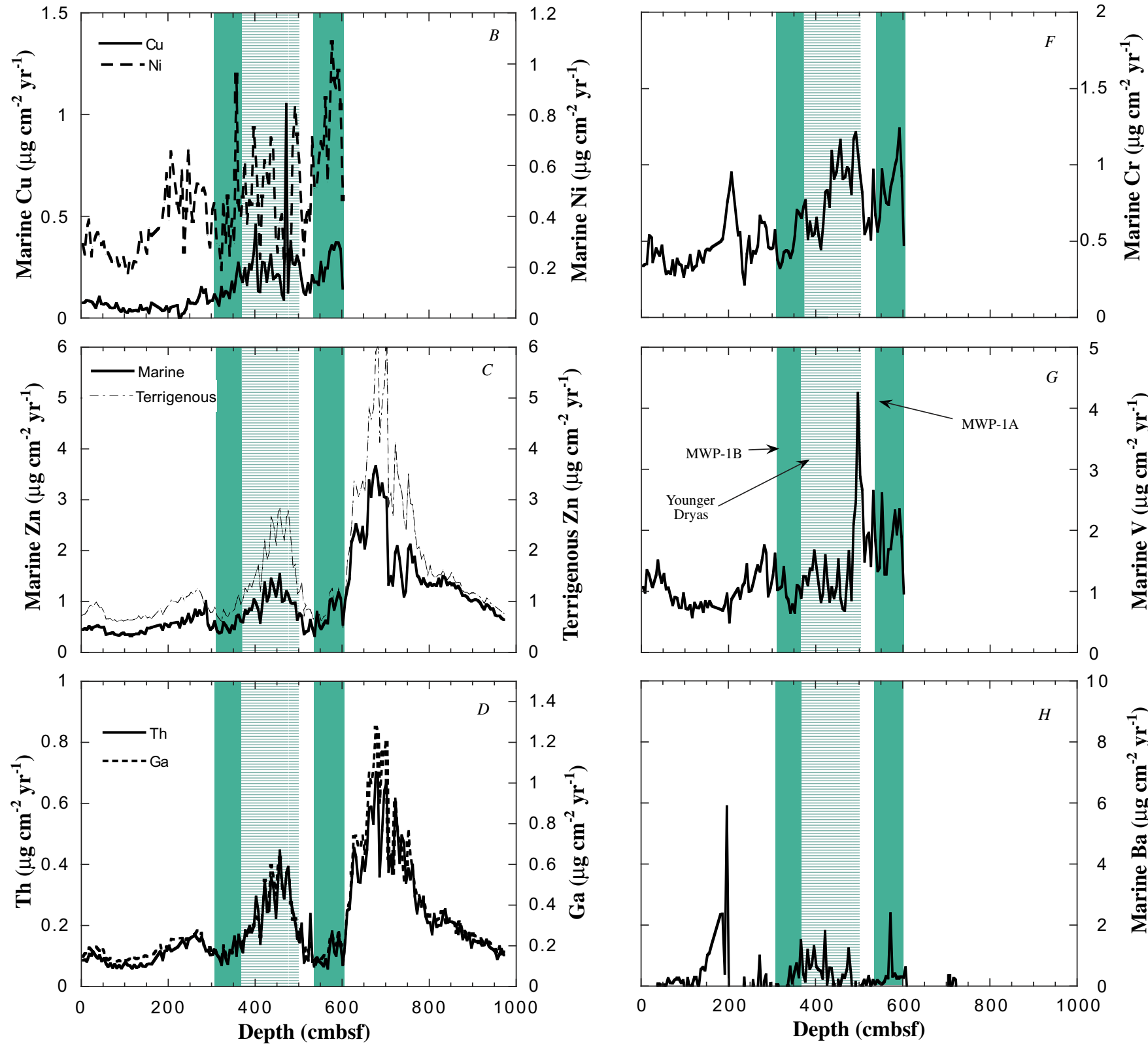

Calendar Age (ka)

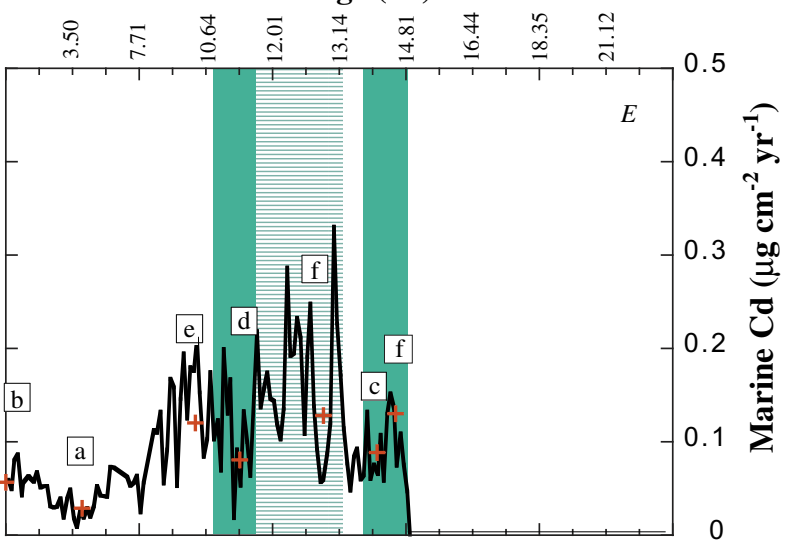

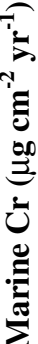
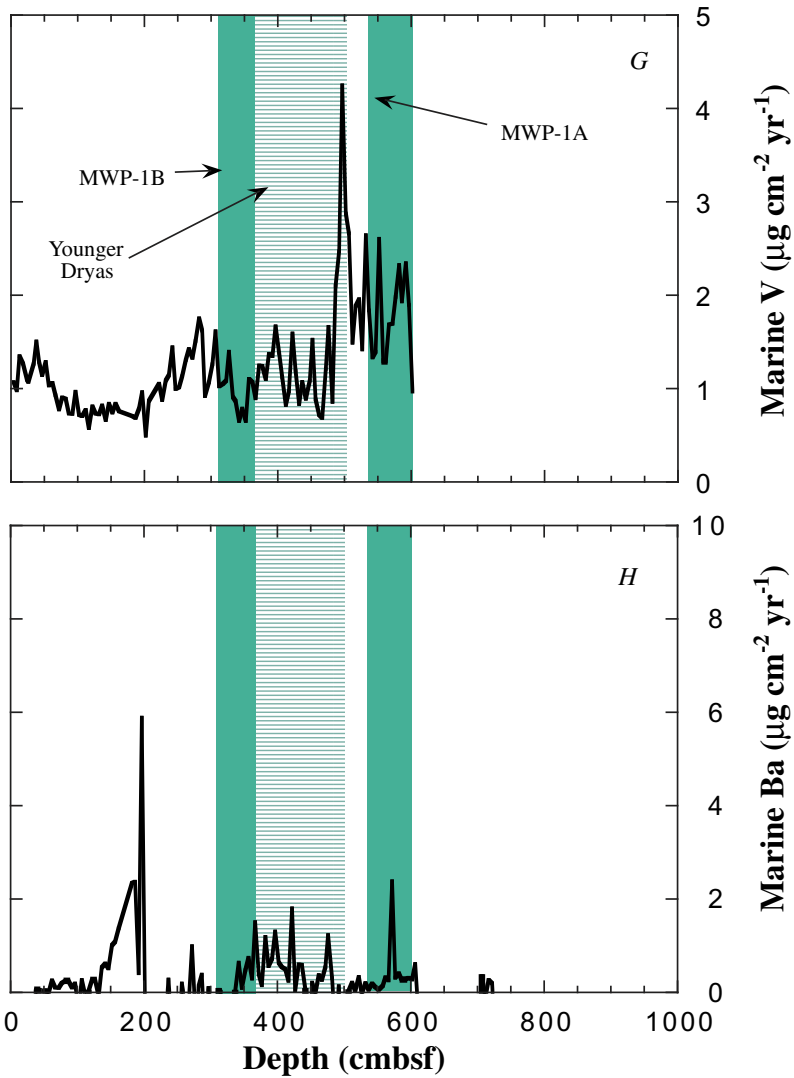

Figure 12. Measured accumulation rates of the terrigenous and marine fractions of trace elements versus depth in core PL07-39PC. Note the different $y$-axis scales. Shaded areas represent the Younger Dryas event and melt-water pulses IA and IB (Fairbanks, 1989). Model ages are taken from Lin and others (1997) and Hughen and others (1998) (fig. 3). Boxed letters in $A$ and $E$ are keyed to model calculations and footnotes in table 7 . Crosses give actual calculated values. 
The removal of $\mathrm{V}$ from $\mathrm{SO}_{4}{ }^{2-}$-reducing bottom water is complex (fig. 10B), such that a change in its accumulation rate from bottom water, in response to a change in $\mathrm{H}_{2} \mathrm{~S}$ concentration, is not possible to predict. $\Delta \mathrm{V}$ is higher in the Cariaco Basin than in Lake Nitinat and Framvaren Fjord, both of which have significantly higher $\mathrm{H}_{2} \mathrm{~S}$ concentrations in the water column at depth (fig. 10B). Even so, the current 65 percent removal of $\mathrm{V}$ from the bottom water in the Cariaco Basin (fig. 10B) contributes to a calculated accumulation-rate sum for the surface sediment that agrees to within 80 percent of the measured rate (table 6).

\section{2 to 14.8 ka (bioturbated sediment)}

Interpretation of the geochemistry of the sediment record between 22 and $17 \mathrm{ka}$ is complicated by the shelf having been exposed, or entirely within the photic zone, making the basin environment far different from what it is today and from what it was like over the past approximately $17 \mathrm{ka}$. Water from the Caribbean Sea now enters the Cariaco Basin across two sills, one at about 120-m depth in the northern part of the basin, east of Tortuga Island, and one at about 146-m depth at the western end of the basin (fig. 1). The latter provided only a shallow connection with the Caribbean Sea at the maximum glacial low stand between 25 and $20 \mathrm{ka}$, when sea level was $120 \mathrm{~m}$ below its current level (Fairbanks, 1990). The foraminiferal record (Peterson and others, 1991; Lin and others, 1997) shows that the basin was not totally isolated. Nonetheless, near isolation of the basin could have had a significant impact on the flux of trace elements into the basin and their accumulation onto the sea floor. One of several possible impacts could have been that the chemical composition of surface sea water and fresh water entering the basin had a dissolved trace-element concentration that was quite different from seawater likely entering at depth currently. This condition makes somewhat tenuous any interpretation of the geochemistry of the bioturbated section of the sediment, prior to about $17 \mathrm{ka}$.

Following the LGM, eastern Mexico, Central America, and Venezuela were cool and arid due to a shift in the ITCZ, perhaps to its present winter position (Bradbury, and others, 1981; Bradbury, 1997; Fritz and others, 2001). Much of the region was covered by dry grasslands (Markgraf, 1989, 1993). Expansion of neotropical savannas, such as the Orinoco Llanas of Venezuela, reflected the dryer conditions (Behling and Hooghiemstra, 2001). Cool conditions may have resulted from low sea-surface temperatures, a result of large volumes of glacial melt water flowing down the Mississippi River (for example, Overpeck and others, 1989). Lin and others (1997) measured the oxygen-isotope record of foraminifera that suggested sea-surface temperatures were as much as $4^{\circ} \mathrm{C}$ cooler, but interpreted the record as reflecting intense upwelling, rather than elevated glacial melt water, that may, nonetheless, not have supported high primary productivity. The dominance of the foraminifera Globigerina ruber over G. bulloides in the bioturbated sediment lead Peterson and others (1991) to conclude that primary productivity, indeed, was lower. Accumula- tion rates of $\mathrm{Cd}$ (fig. 12E) also favor low primary productivity, but the problem of the interpretation of its accumulation prior to $15 \mathrm{ka}$ must be recognized.

The rise in sea level, beginning about $20 \mathrm{ka}$, coincided with an increase in the accumulation of Th and Ga beginning at the end of the LGM and continuing to $16.2 \mathrm{ka}$ (fig. 12). This increase possibly reflected reworking of previously deposited sediment on the shelf by a transgressive ocean. The occurrence at $700 \mathrm{cmbsf}$ of a fine-grained layer and of $\delta^{18} \mathrm{O}$-depleted foraminiferal calcite led Lin and others (1997) to conclude there was a "freshwater event" at this time. A water column of low salinity suggests that, even at this relatively recent time, connection with the Caribbean Sea was quite different from its character of today. It also suggests elevated erosion in the terrestrial environment and elevated accumulation of terrigenous debris. However, arid conditions apparently prevailed in central and northern South America (Lin and others, 1997), although the period corresponds to extremely wet conditions in the western United States. As examples, maximum lake levels in the Bonneville and Lahontan lake systems (Benson, 1991; Thompson and others, 1993) and increased runoff to the Pacific Ocean along central California (Gardner and others, 1997) were achieved at approximately this time.

The oxygen isotope data of Lin and others (1997) also show a convergence of $\delta^{18} \mathrm{O}$ values in the surface-dwelling $G$. ruber and the deeper-dwelling Neogloboquadrina dutertrei during this time, from which they concluded that the watercolumn was well mixed. The low accumulation rate of marine Mo between 610 and 700 cmbsf (figs. 2 and 12) and the bioturbated texture of the sediment further indicate that advection of oxic water into the deeper part of the basin prevented the establishment of $\mathrm{SO}_{4}^{2-}$ reduction in the bottom water.

$\mathrm{Mn}$ is mildly enriched in the sediment from the bioturbated section below 760-cm depth (fig. 7). As Mn deposition from seawater is greatly enhanced under oxic conditions (Landing and Bruland, 1987), its distribution supports the interpretation of deposition of this sediment under oxic bottom-water conditions. A Mn enrichment is also seen in the basal sediment of the distinctly laminated unit. One possible source of this enrichment is the bioturbated sediment, via diagenetic migration of Mn up core at some time following its deposition and reprecipitation possible as a carbonate. Thomson and others (1996) have described in some detail the diagenetic behavior of $\mathrm{Mn}$ in the pelagic environment of the Atlantic Ocean. Oxidizing, Mn-enriched deep-sea sediment in otherwise reducing, Mn-depleted sediment also has been described from the Bering Sea (Gardner and others, 1982) and the North Pacific Ocean (Dean and others, 1989).

Oxic, bottom-water conditions might also account for the slight enrichment of Co (fig. 6; table 1) in these samples, an element that accumulates with Mn under oxic conditions. Some fraction of $\mathrm{Cu}, \mathrm{Ni}$, and $\mathrm{Zn}$ in the bioturbated unit might have had a similar origin as they too can accumulate under oxic conditions, albeit at a very slow rate, as in the pelagic environment. The accumulation rates of the marine fraction of Mo and of the bulk Cd are below detection at the close of this time period. Their absence in this sediment reflects their lack of 
precipitation/adsorption under oxic conditions and possible loss of biogenic debris and its trace-element inventory upon oxidation of the organic matter at and near the surface of the sea floor.

\section{8 to 10.4 ka (distinctly laminated sediment)}

The cool, dry conditions that characterized terrestrial environments around the Caribbean Sea at the close of the LGM continued through the Younger Dryas (Hodell and others, 1991). However, dramatic changes in marine conditions began abruptly at $14.8 \mathrm{ka}$ with sea-level rise that accompanied melt-water pulse (mwp) IA. The rise to dominance of the foraminifera Globigerina bulloides over G. ruber (Peterson and others, 1991) and the increase in the Cd accumulation rate (fig. 12) indicate increased primary productivity. The increase probably reflected the penetration of nutrient-enriched water from the Caribbean Sea across the shelf into the photic zone of the Cariaco Basin. The occurrence of distinctly laminated sediment and increase in the accumulation rate of Mo (fig. 12) further indicate the introduction of $\mathrm{H}_{2} \mathrm{~S}$ in the bottom water.

The accumulation rate of marine Mo was high throughout this period, exhibiting prominent maxima of $4 \mu \mathrm{g} \mathrm{cm}^{-2}$ $\mathrm{yr}^{-1}$ centered at $580 \mathrm{cmbsf}$ and $480 \mathrm{cmbsf}$. Its rate was highly variable throughout the time period, but with its mean accumulation rate decreasing more or less uniformly from the maximum at $480 \mathrm{cmbsf}$ to $1.2 \mu \mathrm{g} \mathrm{cm}^{-2} \mathrm{yr}^{-1}$ near the end of the period. Bottom water, clearly, was $\mathrm{SO}_{4}{ }^{2-}$ reducing throughout this time.

The Mo maxima correspond approximately to major hydrographic and climatic events during this glacial-Holocene transition. The marine Mo maximum at $580 \mathrm{cmbsf}$ (14.4 ka) occurs within mwp-IA (Fairbanks, 1990), whereas the maximum at $480 \mathrm{cmbsf}$ (13.0 ka) corresponds to the onset of the Younger Dryas event. One result of the melt-water pulse was the lowering of surface-water salinity in the Gulf of Mexico, probably leading to an increase in water-column stability. If this same condition extended into the Caribbean Sea, the increased stability of the water column, that is, decrease in bottom-water advection, in conjunction with the increase in primary productivity (Peterson and others, 1991), could have contributed to the establishment of $\mathrm{SO}_{4}{ }^{2-}$-reducing conditions in the bottom water. The increase in primary productivity is reflected by the increase in the $\mathrm{Cd}$ accumulation rate (fig. 12E).

The minimum Mo accumulation rate at the end of this period occurs during mwp-IB, in sharp contrast to its maximum accumulation rate during mwp-IA. Yet, the $\mathrm{Cd}$ accumulation rate was similar for the two melt-water pulses. The dissimilar responses of the two elements during the meltwater pulses suggests a certain level of independence between the rates of bottom-water advection and upwelling into the photic zone. The greater rise of sea level up to the time of mwp-IB than up to the time of mwp-IA may have diminished the effect that a melt-water pulse had on bottom-water advection in the Cariaco Basin. The residence time of bottom water simply continued to increase into mwp-IB, to as much as $250 \mathrm{yr}$ (table 7), following a trend begun toward the end of the Younger Dryas. Upwelling, on the other hand, achieved an intermediate level of approximately $825 \mathrm{~g} \mathrm{C} \mathrm{m}^{-2} \mathrm{yr}^{-1}$ during both events.

The marine $\mathrm{Cd}$ accumulation rate was highly variable throughout this time, although much higher, on average, than the current level (fig. 12E). During the Younger Dryas, its mean accumulation rate was approximately twice as great as during either melt-water pulse. The zooplankton composition (Peterson and others, 1995) suggests a level of primary productivity during mwp-IB that was comparable to the current level, higher than during mwp-IA, but lower than during the Younger Dryas. These relative differences roughly approximate the observed differences in the mean accumulation rate of Cd (fig. 12E).

The model (table 7) underestimates the accumulation rate of $\mathrm{Cd}$ (fig. $12 E$ ), when primary productivity was high, for example, at the onset of the younger Dryas. The interpretation used here of figure 11 from Müller-Karger and others (2001) apparently underestimates the percentage of organic matter exported into the bottom water at times of high primary productivity. Alternatively, the $\mathrm{Cd}$ concentration in the bottom-water source, the $\mathrm{Cd}$ concentration currently in the water between 100- and 250-m depth, may have been underestimated. Both explanations are plausible. The concentrations of $\mathrm{Cd}$ and other nutrient elements in the water column at 100- to 250-m depth are determined largely by the oxidation of organic matter settling through this section of the water column. The elevated level of primary productivity would have supplied an equally elevated load of nutrients out of the photic zone to this intermediate depth. Eventual advection of this water into the bottom water would have supplied a higher flux of $\mathrm{Cd}$ to precipitate as a sulfide (hydrogenous fraction). However, export of a greater percentage of primary productivity out of the photic zone and into the bottom water (biogenic fraction), at the higher level of primary productivity, has the potential of having had a much greater impact (table 6). One possible mechanism of achieving a higher percentage of primary productivity settling into the bottom water, than given by figure 11 of Müller-Karger and others (2001), may have been the occurrence of a greater frequency of pulses in primary productivity than has been observed during the past few years, of an intensity that roughly equaled the event that clogged all sediment traps throughout the water column in May of 1996.

The accumulation rate of terrigenous debris, as recorded by the accumulation rates of $\mathrm{Ga}$ and $\mathrm{Th}$ (fig. 12D), also shows strong variation. The two melt-water pulses are marked by minima in the accumulation rate of the terrigenous fraction. The entrapment of sediment on the shelf apparently dominated any resuspension of shelf sediment by the transgressive sea, as occurred during the initial rise in sea level at the end of the LGM. The flux of the terrigenous fraction shows a maximum during the Younger Dryas event. An increased flux of sediment from local rivers, or of sediment from the Orinoco and Amazon Rivers, may have accounted for this major pulse in the terrigenous accumulation rate. The lake Valencia sediment record suggests a wetter climate at about $10{ }^{14} \mathrm{C}$ ka in northern 
Venezuela than the period following (Bradbury and others, 1981), but the record, unfortunately, does not extend back to the period prior to the Younger Dryas. A summary by Leyden (1995) of data from three sites in Guatemala and Costa Rica suggests that precipitation increased throughout deglaciation, due to strong monsoon conditions.

In summary, the high and strongly variable accumulation rate of Cd during this time indicates a robust rate of upwelling that exhibited sharp temporal variations. A similar pattern for Mo likely reflects a mean residence time of bottom water that was a few tens of years, rather than hundreds of years. A possibly colder and less stable global climate than currently could have contributed to the accumulation-rate patterns of the two trace elements.

\section{4 to 0 ka (faintly laminated sediment)}

Sea level rose steadily by $25 \mathrm{~m}$ during the Holocene (Fairbanks, 1989). Marine conditions within the area might have been relatively stable, compared to the preceding 4.4 kyr. However, both Mo and Cd show strong maxima at 9.9 $\mathrm{ka}$, sharp decreases between $9 \mathrm{ka}$ and $8.6 \mathrm{ka}$, and gradual decreases after $8.4 \mathrm{ka}$ to their lowest accumulation rates of the last $15 \mathrm{kyr}$, at $4.0 \mathrm{ka}$. Both elements exhibit a smooth increase in accumulation rate to about $0.70 \mathrm{ka}$, before decreasing slightly to current levels.

The high $\mathrm{Cd}$ accumulation rate near the very beginning of this period, centered at $9.9 \mathrm{ka}$ (fig. 12E), required an increase in primary productivity, following its lower accumulation rate during the early part of mwp-IB (table 7). The increase in flux of organic matter into the bottom water alone would have increased the concentration of $\mathrm{H}_{2} \mathrm{~S}$, thus increasing the removal of Mo from the bottom water. The lower amplitude variation in the accumulation rate of Mo across this high, for approximately $2.0 \mathrm{kyr}$, than was characteristic of its accumulation during the preceding approximately $4.4 \mathrm{kyr}$ (fig. 12A), suggests a longer bottom water residence time than characterized the preceding period of time, during which it was a few tens of years (table 7). The stable low accumulation rate of Mo after 8.0 ka suggests that the bottom-water residence time continued to increase, in the range of several hundred years. Thus, an increase in bottom-water residence time, coupled with a decreasing level of primary productivity, led to the decreasing accumulation rates of the marine fraction of most trace elements at $8.4 \mathrm{ka}$, following their high rates at $9.9 \mathrm{ka}$.

The change of primary producers from siliceous diatoms to calcareous nannoplankton at approximately $11 \mathrm{ka}$, possibly driven by a reduction in primary productivity (Werne and others, 2000), that is, upwelling and nutrient availability, corresponded to the change in lithology from distinctly laminated to faintly laminated, not to the decrease in the Cd accumulation rate between 8.6 and $8.4 \mathrm{ka}$. Somewhat contrary to the change in primary producers, but in agreement with the accumulation rate of $\mathrm{Cd}$, Peterson and others (1991) interpreted the distribution of $N$. dutertrei as indicating sustained high primary productivity into the upper part of the faintly laminated sediment. The foraminiferal record and $\mathrm{Cd}$ accumulation both suggest a slightly lower level of primary productivity during the maximum that preceded the sharp decrease than during the Younger Dryas period, although the $\mathrm{Cd}$ record during the Younger Dryas is characterized by large variations, between 0.3 and $0.08 \mu \mathrm{g} \mathrm{cm}^{-2} \mathrm{yr}^{-1} \mathrm{Cd}$, a seemingly unstable time.

The pattern of trace-element accumulation rates since 8.4 ka could have been driven by a wetter climate pattern in early to mid-Holocene time in the Caribbean Sea area (Hodell and others, 1991) and increased water-column stability. Lake Valencia, northern Venezuela, was shallow and saline prior to $11 \mathrm{ka}$ (Bradbury and others, 1981; Bradbury, 1997). The lake began to fill by $11 \mathrm{ka}$ and was full and overflowing by about $8.8 \mathrm{ka}$. Thereafter, the lake remained fresh. During the early Holocene, dry scrub vegetation in the lake Valencia basin was replaced by forests (Leyden, 1985). By 8 ka lake sites in eastern Mexico, Central America, Haiti, and elsewhere in Venezuela also were wetter than today (Fritz and others, 2001; Hodell and others, 1991). Hodell and others (1991) concluded from ${ }^{18} \mathrm{O} /{ }^{16} \mathrm{O}$ ratios in ostracod shells from Lake Miragoane, Haiti, that the lake level began to increase about $8 \mathrm{ka}$ and remained high until about $3.2 \mathrm{ka}$. They observed that the inferred lake-level record resembled changes in the annual precipitation cycle as estimated by the seasonal insulation difference at the top of the atmosphere at $10^{\circ} \mathrm{N}$ between August and February. Lake levels in the circum-Caribbean region remained high until about 3.5 $\mathrm{ka}$, marking the beginning of a late Holocene dry phase.

Wetter conditions during the early to middle Holocene also should have been accompanied by a higher influx of terrigenous material. Haug and others (2001) found increases in concentrations of $\mathrm{Fe}$ and $\mathrm{Ti}$ in sediment deposited in the Cariaco Basin between $10 \mathrm{ka}$ and $5 \mathrm{ka}$, in a core from Ocean Drilling Program (ODP Site 1002 at a water depth of $893 \mathrm{~m}$ ). They attributed these increases to be due to a middle Holocene period of increased precipitation and riverine discharge of terrigenous debris. Our data for Fe and Ti also show maxima in lower to mid-Holocene sediments, at $9 \mathrm{ka}$, as do the data for $\mathrm{Ga}$ and $\mathrm{Th}$, our preferred indicators of terrigenous detritus. However, accumulation rates of $\mathrm{Ga}$ and $\mathrm{Th}$ (fig. 12D) indicate that the terrigenous influx was not nearly as great as during the LGM and Younger Dryas. They attributed decreases in concentrations of $\mathrm{Fe}$ and $\mathrm{Ti}$ after about $5 \mathrm{ka}$ as due to a southward migration of the ITCZ and attendant lower precipitation over the Cariaco Basin.

The early-to-middle Holocene wet interval in the tropics contrasted with the extratropical regions of North America where mid-Holocene aridity (the so-called altithermal) was widespread with strong westerly zonal winds (for example, Dean and others, 1996; Fritz and others, 2001). An abrupt change to dry conditions at $8.2 \mathrm{ka}$, with zonal winds over North America, was attributed by Dean and others (2002) to an abrupt northward migration of the polar front in response to changes in the relative proportions of land, water, and, especially, glacial ice on North America during the early Holocene. This change in atmospheric circulation over North America also may have been coupled to the atmospheric circulation over the Atlantic Ocean and (or) thermalhaline circulation within the Atlantic Ocean, as suggested by Shuman and others 
(2002). A wetter mid-Holocene indicates that the ITCZ had moved north of the geographical equator to its present summer position, moving the easterly trade winds farther north and bringing more precipitation to the Caribbean region and reduced upswelling to the Cariaco basin. The reduced upwelling and attendant lower productivity are indeed suggested by the extremely low $\mathrm{Cd}$ and Mo accumulation rates in the midHolocene sediments at about $4.0 \mathrm{ka}$ (figs. 12A, C). The interpretation of wetter conditions, however, seems to conflict with the interpretation here of the minimum Ga snd Th accumulation rates as reflecting day conditions.

A 15 percent increase in primary productivity, following the mid-Holocene minimum, can account for the maxima in $\mathrm{Mo}$ and $\mathrm{Cd}$ accumulation rates at $0.70 \mathrm{ka}$. Such a high would have increased the organic-bound $\mathrm{Cd}$ flux by about 20 per cent, as well as increasing by 20 percent the $\mathrm{H}_{2} \mathrm{~S}$ concentration in the bottom water. At a constant bottom-water residence time, the accumulation rate of Mo would have increased even more. This maximum may be related to the "Little Ice Age" that occurred in the last millennium, but the interpretation clearly requires confirmation from the examination of the sediment record from other basins and a more detailed examination of surface and near-surface sediment from the Cariaco Basin than presented here.

The accumulation rate of Ni following mwp-IB is somewhat different from the other trace elements (fig. 12C). The maximum occurred at a more recent time than the maxima for $\mathrm{Cd}, \mathrm{Mo}$, and $\mathrm{V}$ at $9.9 \mathrm{ka}$. The results presented here seem to offer no explanation for this shift, to well after the maxima of these other trace elements. Ba, which shows a high rate of accumulation at $7.4 \mathrm{ka}$ also needs to be examined in greater detail to ascertain the factors that controlled its accumulation. The accumulation rates of marine $\mathrm{Cu}$ and $\mathrm{Zn}$ show only slight, relatively smooth variations for this entire period of time.

\section{Elemental Ratios and Concentrations- An Alternative Model of Environmental Conditions}

Changes in trace-element ratios in the marine fraction (fig. $13 A$ ) can be interpreted in terms of changes in the intensity of upwelling, that is, primary productivity in the photic zone, relative to changes in the intensity of bottom-water advection, but only in a very general way. The Zn-Mo ratio in the bioturbated sediment closely approaches the curve represented by their concentration ratio in plankton (and terrigenous debris). A shift in a value along the curve reflects the amount of organic matter oxidized and/or diluted by calcite, biogenic $\mathrm{SiO}_{2}$, or terrigenous debris. The rain rate of organic matter into the bottom water, that is, primary productivity, must have been the dominant factor controlling the accumulation of trace-element nutrients from seawater. As bottom-water advection was high enough to maintain bacterial $\mathrm{O}_{2}$ respiration, metals were not reduced to relatively insoluble valence states, the major excep-
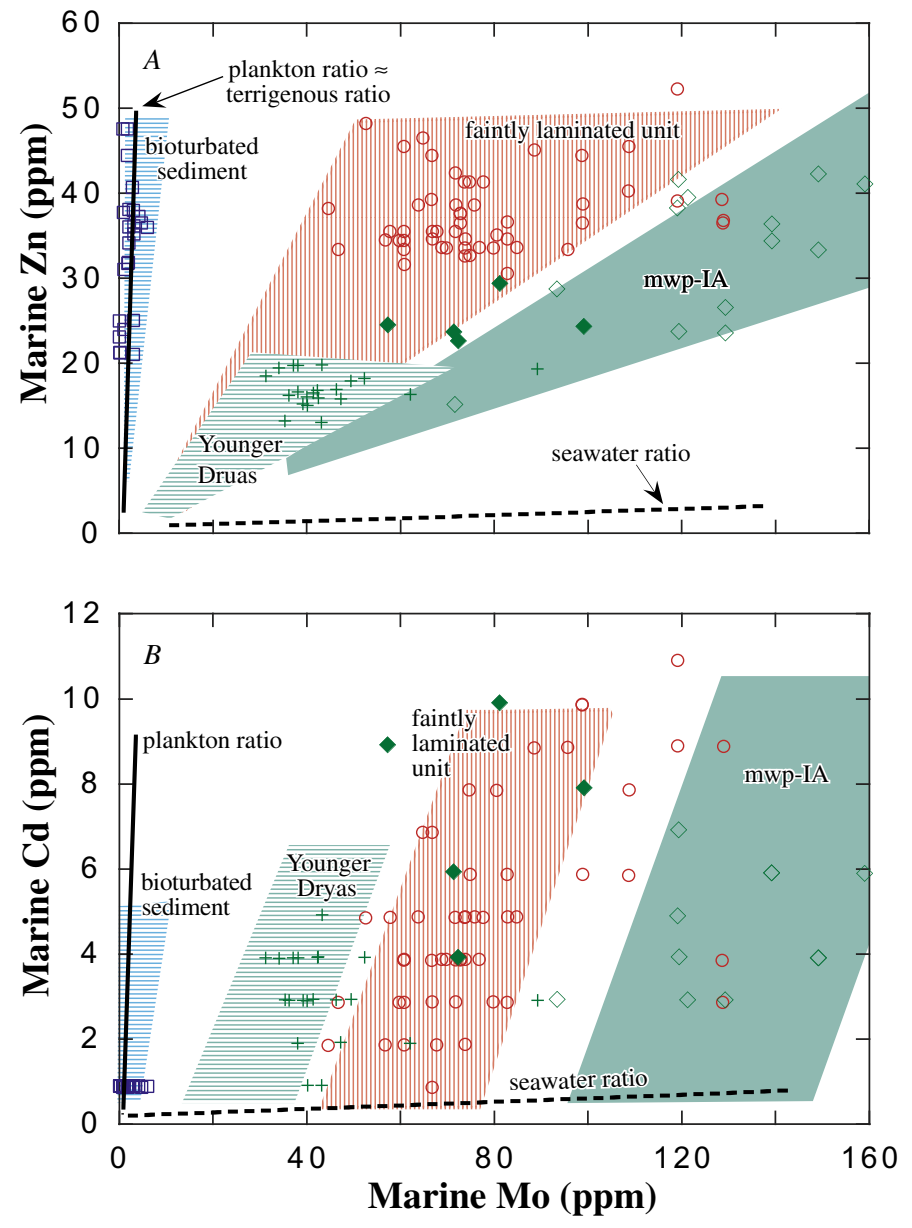

Figure 13. Relations between the concentrations of Mo and $\mathrm{Zn}(A)$ and $\mathrm{Mo}$ and $\mathrm{Cd}(B)$ in the seawater-derived fractions of sediment in core PL07-39PC. Only samples younger than 17 kyr are shown. The broken curve in $A$ represents the $\mathrm{Zn:Mo} \mathrm{ratio} \mathrm{for} \mathrm{seawater} \mathrm{and} \mathrm{the}$ solid curve the ratio for plankton, both extrapolated into the sediment field. Sample symbols are the following: bioturbated unit, blue squares; melt-water pulse IA, green open diamond; melt-water pulse IB green filled diamond; Younger Dryas, green crosses; uppermost $250 \mathrm{~cm}$ of the faintly laminated unit, red circles. The patterned areas are of sediment-core intervals identified in figure 2, the Younger Dryas and melt-water pulses IA and IB.

tion being Mn. Concentrations of Mo and $\mathrm{Zn}$ in samples corresponding to the two melt-water pulses occur within a field that is displaced farthest from the plankton curve and toward the seawater curve. Bottom-water advection and primary productivity combined to give a maximum Mo:Zn ratio of approximately 4. Mo and $\mathrm{Zn}$ accumulation as organic matter at these times was less important than accumulation from bottom water under $\mathrm{SO}_{4}{ }^{2-}$-reducing conditions. However, the accumulation rates of $\mathrm{Zn}$ and $\mathrm{Cd}$ (fig. 12) clearly show that primary productivity was quite high during part of each melt-water pulse. Thus, the ratio does not identify the level of primary productivity. It does suggest that the relation between the upwelling rate and bottom-water advection contributed similarly to traceelement accumulation rates for these two periods.

Sediment that accumulated during the Younger Dryas event has an intermediate Mo: $\mathrm{Zn}$ concentration ratio. The bio- 
logical and bottom-water-redox signals were both important. The low absolute marine $\mathrm{Cd}$ and $\mathrm{Zn}$ concentrations (fig. 2) suggest that primary productivity was lower than at present, but their accumulation rates (fig. 12) clearly required primary productivity to have been much higher during the Younger Dryas.

The Mo:Zn ratios in samples from the faintly laminated sediment occupy the same field as samples from the Younger Dryas. Primary productivity was lower during the period of deposition of this sediment than during the Younger Dryas (fig. 12), yet the concentrations of $\mathrm{Zn}$ and Cd (figs. 2 and 13) suggest the opposite. This relation demonstrates the difficulty of interpreting the sediment record from elemental concentrations alone. Perhaps most important for this study, the distribution of concentration ratios of the marine fraction of trace elements into separate fields defined by lithology lends support to our overall partitioning scheme of trace elements into a terrigenous fraction and the two marine fractions (tables 2 and 6).

The relation between Mo and Cd exhibits a somewhat similar distribution, in that the different lithologic units fall into separate fields, mwp-IB being the exception (fig. 13B). The few samples from this unit occur in the field defined by the faintly laminated unit. However, more samples of this unit need to be analyzed to determine its geochemistry accurately, relative to that of the other units.

An often used alternative procedure to interpret the marine fraction of trace elements is through normalization to $\mathrm{Al}_{2} \mathrm{O}_{3}$, or another measure of the terrigenous fraction of sediment. The use of this procedure can reveal interesting aspects of provenance (Yarincik and others, 2000), when applied to the major-element oxides and trace elements within the terrigenuos fraction alone, but the procedure also identifies the marine fraction. Unfortunately, it obscures interelement relations within the marine fraction of the sediment. For example, the $\mathrm{Mo}: \mathrm{Al}_{2} \mathrm{O}_{3}$ concentration ratio can vary independently of any change in the marine environment, as it is a function of the accumulation rate of the terrigenous fraction, as well as a function of the hydrography of the water column. Also, a Mo: $\mathrm{Al}_{2} \mathrm{O}_{3}$ ratio greater than that of WSA has been considered by others as evidence enough that a sedimentary deposit accumulated under $\mathrm{SO}_{4}{ }^{2-}$-reducing conditions. If the Mo: $\mathrm{Zn}$, or Mo: $\mathrm{Cd}$, ratio of the marine fraction of sediment closely approaches the ratio of plankton, as it does in several organic-carbonenriched sedimentary deposits (Piper, 1994) and in the bioturbated section of this deposit, the bottom water of the basin was unlikely to have been $\mathrm{SO}_{4}{ }^{2-}$ reducing, despite Mo concentrations that are elevated above a terrigenous contribution. Such deposits can have exceptionally high $\mathrm{Mo}: \mathrm{Al}_{2} \mathrm{O}_{3}$ ratios. The procedure advocated here, of examining interelement relations in the marine fraction of sediment alone, allows for unequivocal identification of the bottom water geochemistry. However, it cannot identify either the level of upwelling nor of bottomwater advection in absolute terms.

The distribution of organic carbon also demonstrates the difficulty of identifying the past hydrography of the basin from elemental concentrations. The concentration of organic carbon, or organic matter (table 3 ), in the sediment that accumulated during the Younger Dryas was at its very lowest for the most recent $15 \mathrm{kyr}$ (fig. 14), during the time when primary productivity was at its very highest (fig. $12 E$ ). These low concentrations reflect the relatively high accumulation rates of terrigenous debris (fig. 12D) and biogenic $\mathrm{SiO}_{2}$ (Peterson and others, 1995) during the Younger Dryas, that is, dilution by inorganic phases of the organic matter that settled to the sea floor. This interpretation is further supported by the negative relation that characterizes the entire core, between the concentration of organic matter and bulk sediment accumulation rate (table 4). The overall decrease in organic carbon with depth in the core, for which the regression has a standard deviation of 0.65 and is most strongly represented by curves drawn through the concentration extrema (fig. 14), suggests, however, that a combination of dilution and preservation contributed to its distribution. And finally, the roughly parallel relation in the uppermost $300 \mathrm{~cm}$ of the core, between the distribution of organic carbon and accumulation rate of Cd (fig. 12E), shows that primary productivity also was important to the ultimate concentration of organic carbon in this deposit.

\section{Summary and Conclusions}

(1) The terrigenous sediment fraction in the Cariaco Basin, as measured by the distributions of $\mathrm{Ga}$, Th, and $\mathrm{Al}_{2} \mathrm{O}_{3}$, recorded high accumulation rates that corresponded to the rise in sea level at the end of the last glacial maximum (LGM), beginning at approximately 21 to $18 \mathrm{ka}$. A second high corresponded to the Younger Dryas cold event. The first set of high values possibly reflects resuspension of previously exposed shelf sediment and its re-deposition within the basin, in response to the transgression of the ocean across the shelf following a long period of exposure. The second may reflect an increase in the rate of erosion in the source areas. The terrigenous sediment record for the Holocene seems to agree only moderately with lake records of the Caribbean region,

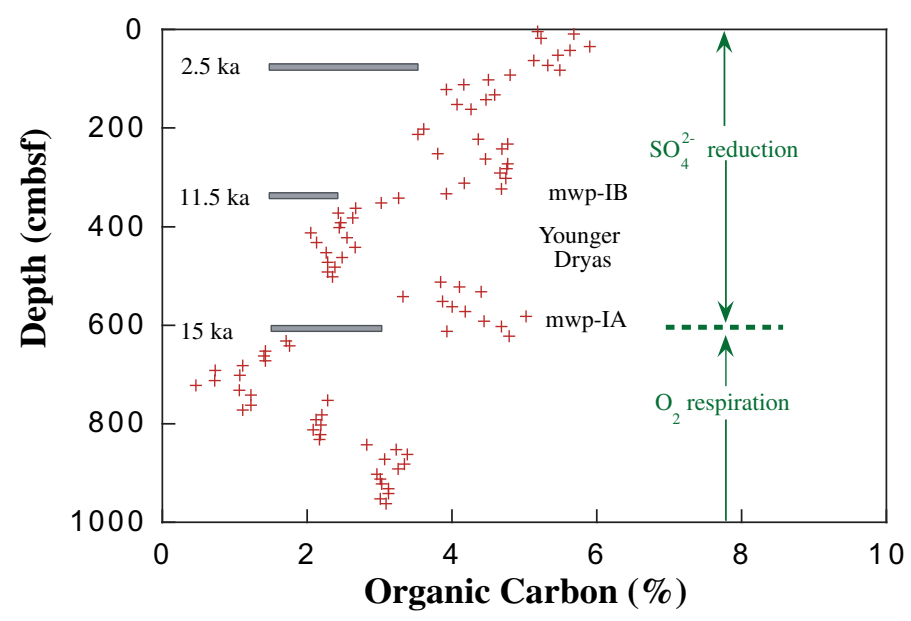

Figure 14. Relation between the concentrations of organic carbon $(0.5 \times$ organic matter) and sediment depth in piston core PL07-39PC, in centimeters below the sea floor (cmbsf). 
of wet conditions in early Holocene becoming dry in the mid Holocene.

(2) The massive texture of sediment deposited immediately prior to $14.8 \mathrm{ka}$ indicates that the sediment was bioturbated and the bottom water oxic. A slight marine Mn and Co fraction in this sediment and low values for marine Mo and $\mathrm{Cd}$ at the very top of the section support such redox conditions. The upper most part of this sediment unit may also have provided a small fraction of several trace elements to the very basal part of the overlying laminated unit.

(3) The accumulation rates of seawater-derived Mo and $\mathrm{Cd}$, beginning $14.8 \mathrm{ka}$, show that primary productivity in the photic zone increased sharply and bottom-water chemistry changed from oxic to $\mathrm{SO}_{4}{ }^{2-}$ reducing. Major peaks in their accumulation rates correspond approximately to melt-water pulse IA and the onset of the Younger Dryas cold event. Mean accumulation rates of the two metals were high for the entire period from $14.8 \mathrm{ka}$ to $8.6 \mathrm{ka}$, but both exhibited large, shortterm variations. The temporal variations in accumulation rates of these two elements were driven by a combination of changes in upwelling into the photic zone (primary productivity) and advection of bottom water (bottom-water residence time). On average, primary productivity was high and the residence time of bottom water was a few tens of years.

(4) By 8.4 ka, primary productivity had decreased sharply by as much as a factor of 2 over a period of $1 \mathrm{kyr}$; bottom water residence times increased from a few tens of years, typical of the period $14.8 \mathrm{kyr}$ to $11 \mathrm{ka}$, to as much as several hundred years. Primary productivity then exhibited a gradual decline to $4.0 \mathrm{ka}$, followed by an equally gradual reversal to the present. Diminished primary productivity provided a greatly reduced flux of organic matter to the bottom water from the preceding $4.4 \mathrm{kyr}$. Nonetheless, bacterial respiration was able to maintain $\mathrm{SO}_{4}{ }^{2-}$-reducing conditions in the bottom water up to the present time, owing to the relatively long bottom-water residence times. The relation of the trend in water column advection for this period ( $8 \mathrm{ka}$ to the present) with the trend in climate over North America and the North Atlantic Ocean suggests that the hydrography of the Cariaco Basin was strongly linked to the global climate.

(5) Accumulation rates of $\mathrm{Cd}$, Mo, and the other trace elements examined ( $\mathrm{Cr}, \mathrm{Cu}, \mathrm{Ni}, \mathrm{V}$., and $\mathrm{Zn}$ ) show a slight maximum centered at about $0.70 \mathrm{ka}$, possibly resulting from a moderately elevated level of primary productivity, that is, upwelling rate, that may have reflected cooling during the "Little Ice Age" of the last millennium.

(6) Variations in the Zn:Mo and Cd:Mo concentration ratios provide support for the partitioning scheme used in this study, whereby a nonreactive terrigenous fraction of trace elements is distinguished from a marine fraction that had both a biogenic (photic-zone) source and a hydrogenous (bottomwater) source. Trace-element ratios of the marine fraction alone, within this and ancient deposits, such as black shales, also can identify the type of bacterial respiration of the bottom water under which the deposits accumulated. Unfortunately, the results of this study demonstrate that neither elemental ratios nor absolute concentrations offer help in ascertaining the intensity of upwelling, that is, primary productivity, of these deposits. The overall distribution of organic carbon similarly fails to reflect solely the changes in primary productivity. In order to identify this property of deposition, it is necessary to have measured or, at the very least, to be able to estimate sediment accumulation rates.

\section{References}

Baedecker, P.A., ed., 1987, Geochemical methods of analysis: U.S. Geological Survey Bulletin, v. 1770, 129 p.

Baines, S.B., Pace, M.L., and Karl, D.M., 1994, Why does the relationship between sinking flux and planktonic primary productivity differ between lakes and oceans: Limnology and Oceanography, v. 39, p. 213-226.

Behling, H., and Hooghiemstra, H., 2001, Neotropical savanna environments in space and time - Late Quaternary interhemispheric comparisons, in Markgraf, V., ed., Interhemispheric climate linkages (present and past interhemispheric climate linkages in the Americas and their societal effects): Academic Press, London, p. 307-323.

Benson, L.V., 1991, Timing of the last highstand of Lake Lahontan: Journal of Paleoclimatology, v. 5, p. 115-126.

Boyle, E.A., Sclater, F.R., and Edmond, J.M., 1976, On the marine geochemistry of cadmium: Nature, v. 263, p. 42-44.

Boyle, E.A., Sclater, F.R., and Edmond, J.M., 1977, The distribution of dissolved copper in the Pacific: Earth and Planetary Science Letters, v. 37, no. 1, p. 38-54.

Bradbury, J. P., 1997, Sources of glacial moisture in Mesoamerica: Quaternary International, v. 43/44, p. 97-110.

Bradbury, J.P., Leyden, B., Salgado-Labouriau, M, and others, 1981, Late Quaternary environmental history of Lake Valencia, Venezuela: Science, v. 214, p. 1299-1305.

Brewer, P.G., and Spencer, D.W., 1974, Distribution of some trace elements in the Black Sea and their flux between dissolved and particulate phases, in Ross, D.A., and Degens, E.T., eds., The Black Sea--geology, chemistry and biology: American Association of Petroleum Geologists, Memoir 20, Tulsa, p. 137-143.

Bruland, K.W., 1983, Trace elements in sea-water, in Riley, J.P., and Chester, R., eds., Chemical oceanography: v. 8, p. 158-220.

Bruland, K.W., and Franks, R.P., 1983, Mn, Ni, Cu, Z, and Cd in the western North Atlantic, in Wong, C.S., Boyle, E., Bruland, K.W., Burton, J.D., and Goldberg, E.D., eds., Trace metals in seawater: Plenum Press, New York, p. 395-414.

Brumsack, H.J., 1986, The inorganic geochemistry of Cretaceous black shales (DSDP Leg 41) in comparison to modern upwelling sediments from the Gulf of California, in Summerhayes, C.P., and Shackleton, N.J., eds., North Atlantic paleoceanography: Geological Society of London, Special Publication 21, p. 447-462.

Clarke, F.W., 1924, Data of geochemistry: U.S. Geological Survey Bulletin 770 .

Clayton, T., Pearce, R.B., and Peterson, L.C., 1999, Indirect climate control of the clay mineral composition of Quaternary sediments from the Cariaco Basin, northern Venezuela: Marine Geology, v. 161, p. 191-206.

Codispoti, L.A., 1980, Temporal nutrient variability in three different upwelling regimes, in Richards, F.A., ed., Coastal upwelling: Vol. I of Coastal and estuarine science, Washington, D.C., American Geophysical Union, p. 209-220. 
Collier, R., 1985, Molybdenum in the Northeast Pacific Ocean: Limnology and Oceanography, v. 30, p. 1351-1354.

Collier, R., and Edmond, J., 1984, The trace element chemistry of marine biogenic particulate matter: Progresses in 0ceanography, v. 13, p. 113-119.

Copin-Montegut, C., and Copin-Montegut, G., 1983, Stoichiometry of carbon, nitrogen, and phosphorus in marine particulate matter: Deep-Sea Research, part A, v. 30, p. 31-46.

Crusius, J., Calvert, S., Pedersen, T., and Sage, D., 1996, Rhenium and molybdenum enrichments in sediments as indicators of oxic, suboxic, and sulfidic conditions of deposition: Earth and Planetary Science Letters, v. 145, p. 65-78.

Dean, W.E., Ahlbrandt, T.S., Anderson, R.Y., and Bradbury, J.P., 1996, Regional aridity in North America during the middle Holocene: The Holocene, v. 6, p. 145-155.

Dean, W.E., Forester, R.M., and Bradbury, J.P., 2002, Early Holocene change in atmospheric circulation in the Northern Great Plains: An upstream view of the 8.2 ka cold event: Quaternary Science Reviews, v. 21, p. 1763-1775.

Dean, W.E., Gardner, J.V., and Hemphill, Haley, E., 1989, Changes in redox conditions in deep-sea sediments in the subarctic North Pacific Ocean-possible evidence for North Pacific Deep Water: Paleoceanography, v. 4, p. 639-653.

Dean, W.E., Piper, D.Z., and Peterson, L.C., 1999, Molybdenum accumulation in Cariaco Basin sediment over the last $24 \mathrm{ky}: \mathrm{A}$ record of water-column anoxia and climate: Geology, v. 27, p. 507-510.

Deuser, W.G., 1973, Cariaco Trench—oxidation of organic matter and residence time of anoxic water: Nature, v. 242, p. 601-603.

Dymond, J., 1981, Geochemistry of Nazca Plate surface sediment-an evaluation of hydrothermal, biogenic, detrital, and hydrogenous sources, in Kulm, L.D., Dymond, J., Dasch, E.J., and Hussong, D.M., eds., Nazca Plate_crustal formation and Andean convergence: Geological Society of America Memoir, v. 154, p. 133-173.

Elderfield, H., Hawkesworth, C.J., Greaves, M.J. and Calvert, S.E., 1981, Rare earth element geochemistry of oceanic ferromanganese nodules and associated sediments: Geochimica et Cosmochimica Acta, v. 45, p. 513-528.

Emerson, S.R., Cranston, R.E., and Liss, P.S., 1979, Redox species in a reducing fjord -equilibrium and kinetic observations: DeepSea Research, v. 26, p. 859-878.

Emerson, S.R., and Huested, S.S., 1991, Ocean anoxia and concentrations of molybdenum and vanadium in seawater: Marine Chemistry, v. 34, p. 177-196.

Eppley, R.W., and Peterson, B.J., 1979, Particulate organic-matter flux and plankton new production in the deep ocean: Nature, v. 282, p. 677-680.

Fairbanks, R.G., 1989, A 17,000-year glacio-eustatic sea level recordinfluence of glacial melting rates on the Younger Dryas event and deep-ocean circulation: Nature, v. 342, p. 637-641.

Fairbanks, R.G., 1990, The age and origin of the "Younger Dryas Climate Event" in Greenland ice cores: Paleoceanography, v. 5, p. 937-948.

Francois, R., 1988, A study on the regulation of the concentration of some trace metals ( $\mathrm{Rb}, \mathrm{Sr}, \mathrm{Zn}, \mathrm{Pb}, \mathrm{Cu}, \mathrm{V}, \mathrm{Cr}, \mathrm{Ni}, \mathrm{Mn}$, and $\mathrm{Mo}$ ) in Saanich Inlet sediments, British Columbia: Marine Geology, v. 83, p. 285-308.

Fritz, S.C., Metcalf, S.E., and Dean, W.E., 2001, Holocene climate of the Americas inferred from paleolimnological records, in Markgraf, V., ed., Interhemispheric climate linkages (present and past interhemispheric climate linkages in the Americas and their societal effects): Academic Press, London, p. 241-264.
Gardner, J.V., Dean, W.E., Klies, D.K., and Baldouf, J.G., 1982, A climate-related oxidizing event in deep-sea sediment from the Bering Sea: Quaternary Research, v. 18, p. 91-107.

Gardner, J.V., Dean, W.E., and Dartnell, P., 1997, Biogenic sedimentation beneath the California Current system for the past $30 \mathrm{kyr}$ and its paleoceanographic significance: Paleoceanography, v. 12, p. 207-225.

Goldberg, E.D., 1963, Mineralogy and chemistry of marine sedimentation, in Shepard, F.P., author, Submarine geology: Harper and Row, New York, p. 436-466.

Haug, G. H., Hughen, K. A., Sigman, D. M., Peterson, L. C., and Röhl, U., 2001, Southward migration of the Intertropical Convergence Zone through the Holocene: Science, v. 293, p. 1304-1308.

Helz, G.R., Miller, C.V., Charnock, J.M., Mosselmans, J.F.W., Patrick, R.A.D., Garner, C.D., and Vaughan, D.J., 1996, Mechanism of molybdenum removal from the sea and its concentration in black shales-EXAFS evidence: Geochimica et Cosmochimca Acta, v. 60, p. 3631-3642.

Hodell, D.A., Curtis, J.H., Jones, G.A., Higuera-Gundy, A., Brenner, M., Binford, M.W., and Dorsey, K.T., 1991, Reconstruction of Caribbean climate change over the past 10,500 years: Nature, v. 352, p. 790-793.

Hughen, K.A., Overpeck, J.T., Peterson, L.C., and Anderson, R.F., 1996a, The nature of varved sedimentation in the Cariaco Basin, Venezuela, and its palaeoclimatic significance, in Kemp, A.E.S., ed., Palaeoclimatology and palaeoceanography, from laminated sediments: Geological Society, London, Special Publication, v. 116, p. 171-183.

Hughen K.A., Overpeck, J.T., Peterson, L.C., and Trumbore, S., 1996b, Rapid climate changes in the tropical Atlantic region during the last deglaciation: Nature, v. 380, p. 51-54.

Hughen, K.A., Overpeck, J.T., Lehman, S.R., Kashgarian, M., Southon, J., Peterson, L.C., Alley, R., and Sigman, D.M., 1998, Deglacial changes in ocean circulation from an extended radiocarbon calibration: Nature, v. 391, p. 65-68.

Isaacs, C.M., 1980, Diagenesis in the Monterey Formation examined laterally along the coast near Santa Barbara, California: Ph.D. dissertation, Stanford University, Palo Alto, 329p.

Jacobs, L., and Emerson, S., 1982, Trace metal solubility in an anoxic basin: Earth and Planetary Science Letters, v. 60, p. 237-252.

Jacobs, L., Emerson, S., and Skei, J., 1985, Partitioning and transport of metals across the $\mathrm{O}_{2} / \mathrm{H}_{2} \mathrm{~S}$ interface in a permanently anoxic basin, Framvaren Fjord, Norway: Geochimica et Cosmochimca Acta, V. 49, P. 1433-1444.

Jacobs, L., Emerson, S., and Huested, S.S., 1987, Trace metal chemistry in the Cariaco Trench: Deep-Sea Research, v. 34, p. 965-981.

Komer, B., and Becker, B., 1993, German oak and pine ${ }^{14} \mathrm{C}$ calibration, 7200-9439 BC: Radiocarbon, v. 35, p. 125-135.

Kremling, K., 1983, The behavior of Zn, Cd, Cu, Ni, Co, Fe, and Mn in anoxic Baltic waters: Marine Chemistry, v. 13, p. 87-108.

Landing, W.M., and Bruland, K.W., 1987, The contrasting biochemistry of iron and manganese in the Pacific Ocean: Geochimica et Cosmochimica Acta, v. 51, p. 29-43.

Landing, W.M., and Lewis, B.L., 1991, Thermodynamic modeling of trace metal speciation in the Black Sea, in Izdar, E., and Murray, J.W., eds., Black Sea oceanography: Kluwer Academy Publishers, Dordrecht, p. 125-160.

Leinen, M., 1977, A normative calculation technique for determining opal in deep-sea sediments: Geochimica et Cosmochimca Acta, v. 41, p. 671-676.

Leyden, B.W., 1985, Late Quaternary aridity and Holocene moisture fluctuations in the Lake Valencia basin, Venezuela: Ecology, v. 66, p. $1279-1295$. 
Leyden, B.W., 1995, Evidence of the Younger Dryas in Central America: Quaternary Science Reviews, v. 14, p. 833-839.

Lin, H.-L., Peterson, L.C., Overpeck, J.T., Trumbore, S.E., and Murray, D.W., 1997, Late Quaternary climate change from $\delta^{18} 0$ records of multiple species of planktonic foraminifera-high-resolution records from the anoxic Cariaco Basin, Venezuela: Paleoceanography, v. 12, p. 415-427.

Lyons, T.W., 1997, Sulfur isotopic trends and pathways of iron sulfide formation in upper Holocene sediments of the anoxic Black Sea: Geochimica et Cosmochimica Acta, v. 61, p. 3367-3382

Markgraf, V., 1989, Paleoclimates in Central and South America since 18,000 B.P., based on pollen and lake-level records: Quaternary Science Reviews, v. 8, p. 1-24.

Markgraf, V., 1993, Climate history of Central and South America since 18,000 B.P-comparison of pollen records and model simulations, in Wright, H.E., Jr., Kutzback, J.E., Webb, T. III, Ruddiman, W.F., Street-Perrott, F.A., and Bartlein, P.J., eds., Global climates since the last glacial maximum: University Minnesota Press, Minneapolis, p. 357-385.

Martin, J.K., and Knauer, G.A., 1973, The elemental composition of plankton: Geochimica et Cosmochimica Acta, v. 37, p. 1639-1653.

Medrano, M.D., and Piper, D.Z., 1995, Partition of minor elements and major-element oxides between rock components and calculation of the marine-derived fraction of the minor elements in rocks of the Phosphoria Formation, Idaho and Wyoming: U.S. Geological Survey Open-File Report, v. 95-270, 79pp.

Müller-Karger, F., Varela, R., Thunell, R., Scranton, M., Bohrer, R., Taylor, G., Capelo, J., Astor, Y., Tappa, E., Ho, T.-Y., and Walsh, J.J., 2001, Annual cycle of primary productivity in the Cariaco Basin-response to upwelling and implications for vertical export: Journal of Geophysical Research, v. 106, no., C3, p. 4527-4542.

Murray, J.W., Spell, B., and Paul, B., 1983, The contrasting geochemistry of manganese and chromium in the eastern tropical Pacific Ocean, in Wong, C.S., Boyle, E., Bruland, K.W., Burton, J.D., and Goldberg, E.D., eds., Trace metals in seawater: New York, Plenum, p. 643-669.

Overpeck, J.T., Peterson, L.C., Kipp, N., Imbrie, J., and Rind, D., 1989, Climate change in the circum North Atlantic during the last deglaciation: Nature, v. 338, p. 553-557.

Palmer, M.A., 1985, Rare earth elements in foraminifera tests: Earth and Planetary Science Letters, v. 73, p. 285-298.

Parker, R.A., and Sackett, W.M., 1976, Radiocarbon analysis of sediments from a continental margin anoxic basin-the Cariaco Trench: Geological Society of America Annual Meeting, Abstracts and Programs, v. 8, p. 57.

Pedersen, T.F., Waters, R.D., and MacDonald, R.W., 1989, On the natural enrichment of cadmium and molybdenum in the sediments of Ucluelet Inlet, British Columbia: Science of the Total Environment, v. 79, p. 125-139.

Peterson, L.C., Overpeck, J.T., Kipp, N.G., and Imbrie, J., 1991, A highresolution late Quarter-nary upwelling record from the anoxic Cariaco Basin, Venezuela: Paleoceanography, v. 6, p. 99-119.

Peterson, L.C., Overpeck, J.T., and Murray, D.W., 1995, Anoxic basin records detailed climate history: Drill Bits (JOI/USSAC Newsletter), v. 8, p. 10-13.

Piper, D.Z., 1994, Seawater as the source of minor elements in black shales, phosphorites, and other sedimentary deposits: Chemical Geology, v. 114, p. 95-114.

Piper, D.Z., and Isaacs, C.M., 1994, Geochemistry of minor elements in the Monterey Formation, CA.- seawater chemistry of deposition: U.S. Geological Survey Professional Paper, v. 1566 , p. 1-41.

Piper, D.Z., and Isaacs, C.M., 1996, Instability of bottom-water redox conditions during accumulation of Quaternary sediment in the Japan Sea: Paleoceanography, v. 11, p. 171-190.

Powell, T.G., Cook, P.J., and McKirdy, D.M., 1975, Organic geochemistry of phosphorites-relevance to petroleum genesis: American Association of Petroleum Geologists Bulletin, v. 59, p 618-632.

Redfield, A.C., Ketchum, B.H., and Richards, F.A., 1963, The influence of organisms on the composition of seawater, in Hill, M.N., ed., The Sea: Vol. 2 of Ideas and observations on progress in the study of the seas, John Wiley and Sons, New York, p. 26-77.

Richards, F.A., 1975, The Cariaco Basin (Trench): Oceanography and Marine Biology Annual Reviews, v. 13, p. 11-67.

Richards, F.A., and Vaccaro, R.F., 1956, The Cariaco Trench, an anaerobic basin in the Caribbean Sea: Deep-Sea Research, v. 3, p. 214-228.

Schuffert, J.D., Jahnke, R.A. Kastner, M., Leather, J., Struz, A., and Wing, M.R., 1994, Rates of formation of modern phosphorite off western Mexico: Geochimica et Cosmochimica Acta, v. 58, p. 5001-5010.

Sclater, R.F., Boyle, E., and Edmond, J.M., 1976, On the marine geochemistry of nickel: Earth and Planetary Science Letters, v. 31, no. 7, p. 119-128.

Scranton, M.I., Sayles, F.L., Bacon, M.P., and Brewer, P.G., 1987, Temporal changes in the hydrography and chemistry of the Cariaco Trench: Deep-Sea Research, v. 34, p. 945-963.

Sherrell, R.M., 1989, The trace-element geochemistry of suspended oceanic particulate matter: PhD Dissertation, Massachusetts Institute of Technology, Woods Hole Oceanographic Institute, Woods Hole, 211p.

Thompson, R.S., Whitlock, C., Bartlein, P.J., Harrison, S.P., and Spaulding, W.G., 1993, Climatic changes in western United States since 18,000 B.P, in Wright, H.E., Jr., Kutzback, J.E., Webb, T., III, Ruddiman, W.F., Street-Perrott, F.A., and Bartlein, P.J., eds., Global climates since the last glacial maximum: University Minnesota Press, Minneapolis, p. 468-513.

Thomson, J., Higgs, N.C., and Colley, S., 1996, Diagenetic redistribution of redox-sensitive elements in northeast Atlantic glacial/ interglacial sediments: Earth and Planetary Science Letters, v. 139 , p. 365-377.

Thunell, R.C., Varela, R., Coolister, R.J., Sigman, D., Müller-Karger, F., and Bohrer, R., 2000, Organic carbon fluxes, degradation, and accumulation in an anoxic basin-sediment trap results from the Cariaco Basin: Limnology and Oceanography, v. 45, p. 300308.

Varela, R, Capelo, J.C., Gutierrez, J., Müller-Karger, F., and DiazRamos, J.R., 1997, Primary productivity in Cariaco Basin waters: EOS Transactions of the American Geophysical Union, v. 78, p. F342.

Wedepohl, K.H., ed., 1969-1978, Handbook of Geochemistry, Vol. I-IV: Springer-Verlag, Berlin.

Werne, J.P., Hollander, D.J., and Lyons, T.W., 2000, Climate-induced variations in productivity and planktonic ecosystem structure from the Younger Dryas to Holocene in the Cariaco Basin, Venezuela: Paleoceanography, v. 15, p. 19-29.

Yarincik, K.M., Murray, R.W., and Peterson, L.C., 2000, Climatically sensitive eolian and hemipelagic deposition in the Cariaco Basin, Venezuela, over the past 578,000 years: Results from $\mathrm{Al} / \mathrm{Ti}$ and K/Al: Paleoceanography, v. 15, p. 210-228. 